Measine co and Estimated Sodium-Adsorption Ratios for rongue her and its Tributaries, Montana and Wyoming,

2004010

s.t.

tons

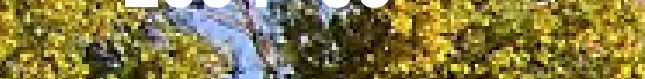

tint

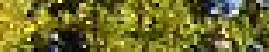

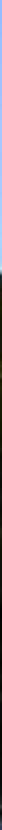

3.

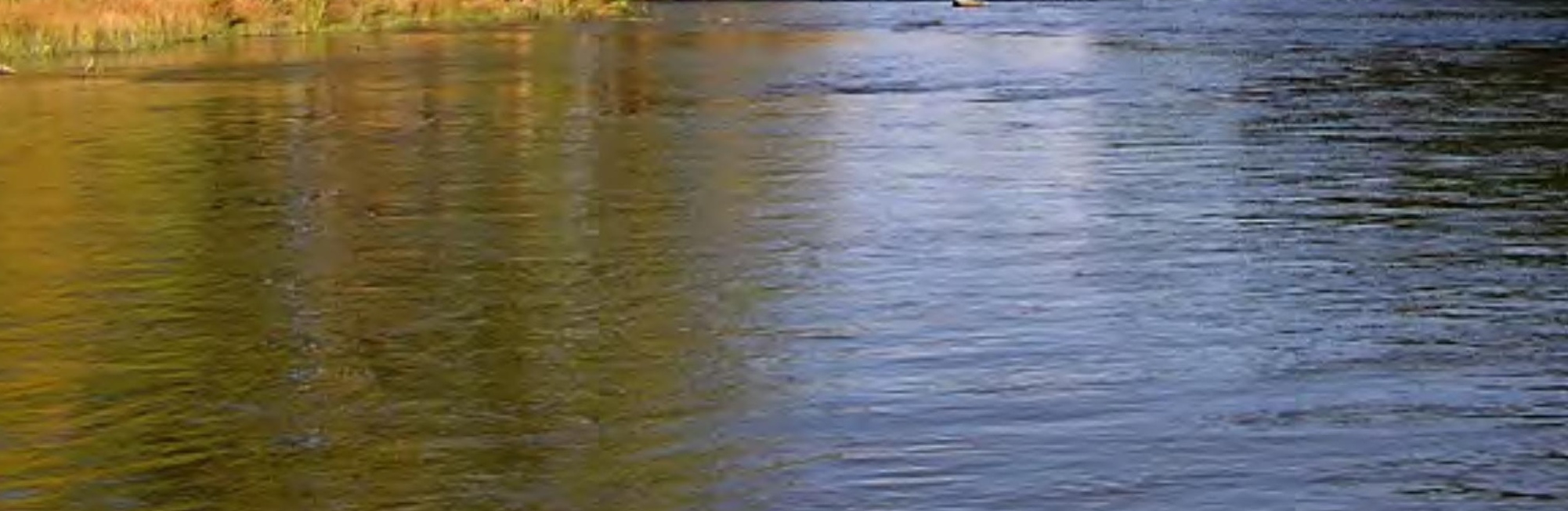

Scientific Investigations Report 2007-5072

U.S. Department of the Interior

U.S. Geological Survey 
COVER PHOTOGRAPH: Tongue River upstream from State line, near Acme, Wyoming. Photograph by Stacy M. Kinsey, U.S. Geological Survey, taken September 27, 2005. 


\section{Measured and Estimated Sodium- Adsorption Ratios for Tongue River and its Tributaries, Montana and Wyoming, 2004-06}

By M.R. Cannon, David A. Nimick, Thomas E. Cleasby, Stacy M. Kinsey, and John H. Lambing

Scientific Investigations Report 2007-5072 


\section{U.S. Department of the Interior DIRK KEMPTHORNE, Secretary}

\section{U.S. Geological Survey \\ Mark D. Myers, Director}

\section{U.S. Geological Survey, Reston, Virginia: 2007}

For product and ordering information:

World Wide Web: http://www.usgs.gov/pubprod

Telephone: 1-888-ASK-USGS

For more information on the USGS--the Federal source for science about the Earth, its natural and living resources, natural hazards, and the environment:

World Wide Web: http://www.usgs.gov

Telephone: 1-888-ASK-USGS

Any use of trade, product, or firm names is for descriptive purposes only and does not imply endorsement by the U.S. Government.

Although this report is in the public domain, permission must be secured from the individual copyright owners to reproduce any copyrighted materials contained within this report.

Suggested citation:

Cannon, M.R., Nimick, D.A., Cleasby, T.E., Kinsey, S.M., and Lambing, J.H., 2007, Measured and estimated sodiumadsorption ratios for Tongue River and its tributaries, Montana and Wyoming, 2004-06: U.S. Geological Survey Scientific Investigations Report 2007-5072, 45 p. 


\section{Contents}

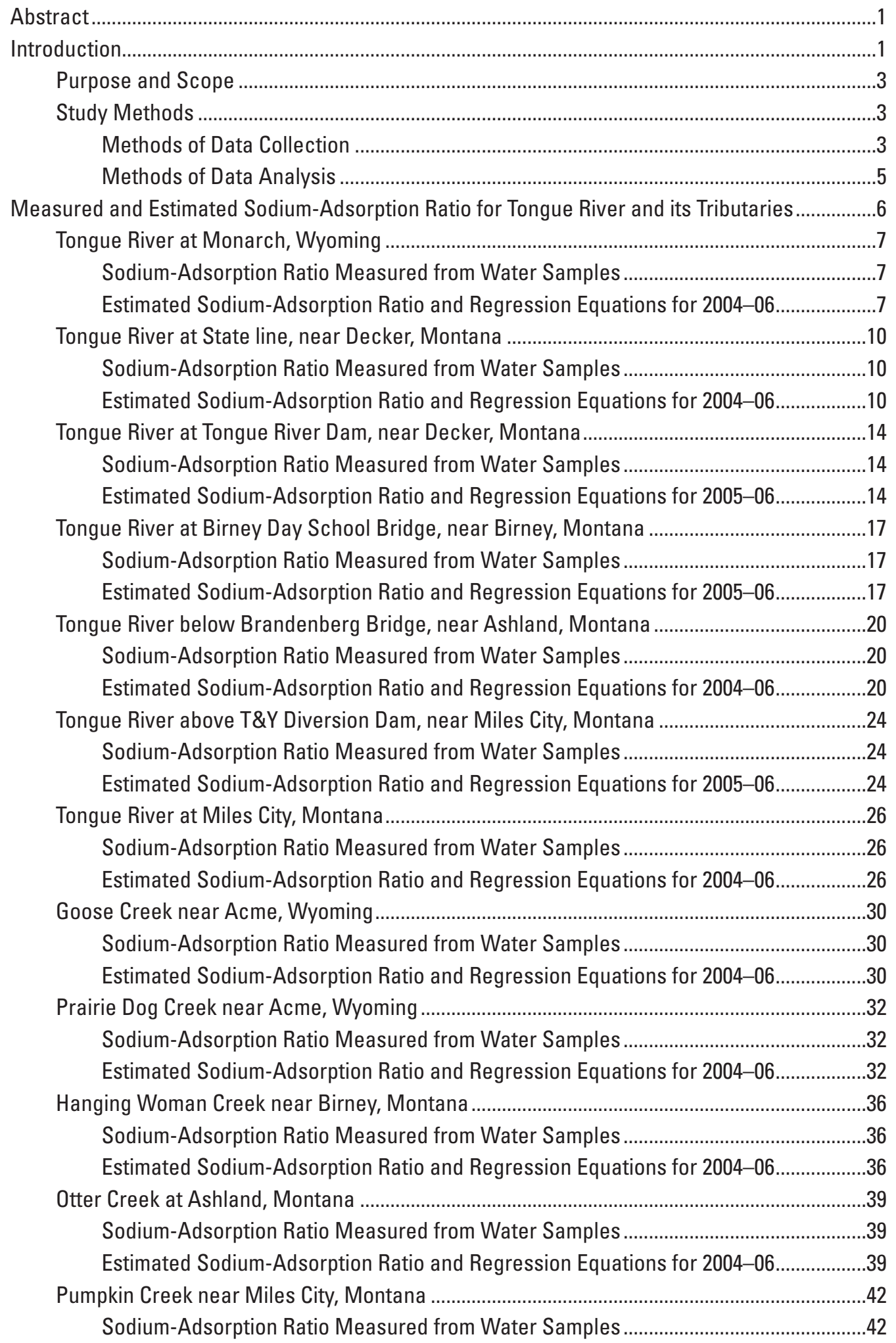




\section{Figures}

1. Map showing Tongue River watershed and location of sampling sites, Montana and Wyoming...

2-24. Graphs showing:

2. Relation of specific conductance (SC) and sodium-adsorption ratio (SAR) for water-quality samples collected at Tongue River at Monarch, Wyoming

3. Sodium-adsorption ratio (SAR) determined from water-quality samples and estimated from specific conductance (SC) for Tongue River at Monarch, Wyoming, using relations shown in figure 2

4. Relation of specific conductance (SC) and sodium-adsorption ratio (SAR) for water-quality samples collected at Tongue River at State line, near Decker, Montana

5. Sodium-adsorption ratio (SAR) determined from water-quality samples and estimated from specific conductance (SC) for Tongue River at State line, near Decker, Montana, using relations shown in figure 4

6. Relation of specific conductance (SC) and sodium-adsorption ratio (SAR) for water-quality samples collected January 2004 through October 2005 at Tongue River at Tongue River Dam, near Decker, Montana, and used to estimate SAR for the 2005 irrigation season.

7. Sodium-adsorption ratio (SAR) determined from water-quality samples and estimated from specific conductance (SC) for Tongue River at Tongue River Dam, near Decker, Montana, for the 2005 irrigation season using relation shown in figure 6

8. Relation of specific conductance (SC) and sodium-adsorption ratio (SAR) for water-quality samples collected January 2004 through October 2005 at Tongue River at Birney Day School Bridge, near Birney, Montana, and used to estimate SAR for the 2005 irrigation season.

9. Sodium-adsorption ratio (SAR) determined from water-quality samples and estimated from specific conductance (SC) for Tongue River at Birney Day School Bridge, near Birney, Montana, for the 2005 irrigation season using relation shown in figure 8

10. Relation of specific conductance (SC) and sodium-adsorption ratio (SAR) for water-quality samples collected at Tongue River below Brandenberg Bridge, near Ashland, Montana

11. Sodium-adsorption ratio (SAR) determined from water-quality samples and estimated from specific conductance (SC) for Tongue River below Brandenberg Bridge, near Ashland, Montana, using the relations shown in figure 10

12. Relation of specific conductance (SC) and sodium-adsorption ratio (SAR) for water-quality samples collected November 2004 through 0ctober 2005 at Tongue River above T\&Y Diversion Dam, near Miles City, Montana, and used to estimate SAR for the 2005 irrigation season. 
13. Sodium-adsorption ratio (SAR) determined from water-quality samples and estimated from specific conductance (SC) for Tongue River above T\&Y Diversion Dam, near Miles City, Montana, for the 2005 irrigation season using relation shown in figure 12

14. Relation of specific conductance (SC) and sodium-adsorption ratio (SAR) for water-quality samples collected at Tongue River at Miles City, Montana.........29

15. Sodium-adsorption ratio (SAR) determined from water-quality samples and estimated from specific conductance (SC) for Tongue River at Miles City, Montana, using relations shown in figure 14

16. Relation of specific conductance (SC) and sodium-adsorption ratio (SAR) for water-quality samples collected at Goose Creek near Acme, Wyoming............31

17. Sodium-adsorption ratio (SAR) determined from water-quality samples and estimated from specific conductance (SC) for Goose Creek near Acme, Wyoming, using relations shown in figure 16.

18. Relation of specific conductance (SC) and sodium-adsorption ratio (SAR) for water-quality samples collected at Prairie Dog Creek near Acme, Wyoming.....

19. Sodium-adsorption ratio (SAR) determined from water-quality samples and estimated from specific conductance (SC) for Prairie Dog Creek near Acme, Wyoming, using relations shown in figure 18.

20. Relation of specific conductance (SC) and sodium-adsorption ratio (SAR) for water-quality samples collected at Hanging Woman Creek near Birney, Montana.

21. Sodium-adsorption ratio (SAR) determined from water-quality samples and estimated from specific conductance (SC) for Hanging Woman Creek near Birney, Montana, using relations shown in figure 20

22. Relation of specific conductance (SC) and sodium-adsorption ratio (SAR) for water-quality samples collected at Otter Creek at Ashland, Montana ..............41

23. Sodium-adsorption ratio (SAR) determined from water-quality samples and estimated from specific conductance (SC) for Otter Creek at Ashland, Montana, using relations shown in figure 22.

24. Specific conductance (SC) and sodium-adsorption ratio (SAR) for water-quality samples collected March 2004 through October 2005 at Pumpkin Creek near Miles City, Montana.

\section{Tables}

1. Site information and summary of the number of water-quality samples collected during various years by the U.S. Geological Survey at sites in the Tongue River surface-water-quality monitoring network....

2. Water-sample data collected at Tongue River at Monarch, Wyoming (06299980), and used in regression equations for estimating sodium-adsorption ratio from specific conductance

3. Water-sample data collected at Tongue River at State line, near Decker, Montana (06306300), and used in regression equations for estimating sodium-adsorption ratio from specific conductance.

4. Water-sample data collected at Tongue River at Tongue River Dam, near Decker, Montana (06307500), and used in regression equations for estimating sodium-adsorption ratio from specific conductance 
5. Water-sample data collected at Tongue River at Birney Day School Bridge, near Birney, Montana (06307616), and used in regression equations for estimating sodium-adsorption ratio from specific conductance

6. Water-sample data collected at Tongue River below Brandenberg Bridge, near Ashland, Montana (06307830), and used in regression equations for estimating sodium-adsorption ratio from specific conductance.

7. Water-sample data collected at Tongue River above T\&Y Diversion Dam, near Miles City, Montana (06307990), and used in regression equations for estimating sodium-adsorption ratio from specific conductance

8. Water-sample data collected at Tongue River at Miles City, Montana (06308500), and used in regression equations for estimating sodium-adsorption ratio from specific conductance

9. Water-sample data collected at Goose Creek near Acme, Wyoming (06305700), and used in regression equations for estimating sodium-adsorption ratio from specific conductance

10. Water-sample data collected at Prairie Dog Creek near Acme, Wyoming (06306250), and used in regression equations for estimating sodium-adsorption ratio from specific conductance.

11. Water-sample data collected at Hanging Woman Creek near Birney, Montana (06307600), and used in regression equations for estimating sodium-adsorption ratio from specific conductance

12. Water-sample data collected at Otter Creek at Ashland, Montana (06307740), and used in regression equations for estimating sodium-adsorption ratio from specific conductance

13. Water-sample data collected at Pumpkin Creek near Miles City, Montana (06308400), and used in regression analysis of specific conductance and sodium-adsorption ratio 


\section{Conversion Factors, Datum, Abbreviated units, and Acronyms}

\begin{tabular}{lcl}
\hline Multiply & By & To obtain \\
\hline foot (ft) & Length & \\
inch (in.) & 0.3048 & meter $(\mathrm{m})$ \\
mile $(\mathrm{mi})$ & 25.4 & millimeter $(\mathrm{mm})$ \\
& 1.609 & kilometer $(\mathrm{km})$ \\
\hline acre & Area & \\
acre & 4,047 & square meter $\left(\mathrm{m}^{2}\right)$ \\
square mile $\left(\mathrm{mi}^{2}\right)$ & 0.004047 & square kilometer $\left(\mathrm{km}^{2}\right)$ \\
square mile $\left(\mathrm{mi}^{2}\right)$ & 259.0 & hectare $($ ha) \\
\hline & 2.590 & square kilometer $\left(\mathrm{km}^{2}\right)$ \\
\hline acre-foot $($ acre- $\mathrm{ft})$ & Volume & \\
\hline & $1,233.5$ & cubic meter $\left(\mathrm{m}^{3}\right)$ \\
\hline cubic foot per second $(\mathrm{ft} 3 / \mathrm{s})$ & Flow rate & \\
\hline
\end{tabular}

Horizontal coordinate information is referenced to North American Datum of 1927 (NAD 27).

Vertical coordinate information is referenced to the National Geodetic Vertical Datum of 1929 (NGVD 29).

Altitude, as used in this report, refers to distance above the vertical datum (NGVD 29) in feet. Specific conductance is given in microsiemens per centimeter at 25 degrees Celsius $(\mu \mathrm{S} / \mathrm{cm}$ at $\left.25^{\circ} \mathrm{C}\right)$.

Abbreviated units used in this report:

$\mu \mathrm{g} / \mathrm{L} \quad$ micrograms per liter

$\mu \mathrm{m} \quad$ micrometer

$\mathrm{mg} / \mathrm{L} \quad$ milligrams per liter

$\mu \mathrm{S} / \mathrm{cm}$ microsiemens per centimeter at 25 degrees Celsius

ppb parts per billion

Acronyms used in this report:

CBM coal-bed methane

NWIS National Water Information System

NWQL National Water Quality Laboratory, Denver, Colorado

RPD relative percent difference

SAR sodium-adsorption ratio

SC specific conductance

USGS U.S. Geological Survey 


\title{
Measured and Estimated Sodium-Adsorption Ratios for Tongue River and its Tributaries, Montana and Wyoming, 2004-06
}

\author{
By M.R. Cannon, David A. Nimick, Thomas E. Cleasby, Stacy M. Kinsey, and John H. Lambing
}

\section{Abstract}

The Tongue River drains an area of about 5,400 square miles and flows northward from its headwaters in the Bighorn National Forest of northeastern Wyoming to join the Yellowstone River at Miles City, Montana. Water from the Tongue River and its tributaries is extensively used for irrigation in both Wyoming and Montana. The Tongue River watershed contains vast coal deposits that are extracted at several surface mines. In some areas of the watershed, the coal beds also contain methane gas (coal-bed methane or natural gas), which has become the focus of intense exploration and development. Production of coal-bed methane requires the pumping of large volumes of ground water from the coal beds to reduce water pressure within the formation and release the stored gas. Water from the coal beds typically is high in sodium and low in calcium and magnesium, resulting in a high sodium-adsorption ratio (SAR). Disposal of ground water with high sodium concentrations into the Tongue River has the potential to increase salinity and SAR of water in the river, and potentially reduce the quality of water for irrigation purposes.

This report documents SAR values measured in water samples collected at 12 monitoring sites in the Tongue River watershed and presents regression relations between specific conductance (SC) and SAR at each site for the years 2004-06. SAR in water samples was determined from laboratorymeasured concentrations of sodium, calcium, and magnesium. The results of regression analysis indicated that SC and SAR were significantly related $(p$-values $<0.05$ ) at most sites. The regression relations developed for most monitoring sites in the Tongue River watershed were used with continuous SC data to estimate daily SAR during the 2004 and 2005 irrigation seasons and to estimate 2006 provisional SAR values, which were displayed on the Web in real-time.

Water samples were collected and analyzed from seven sites on the main stem of the Tongue River located at: (1) Monarch, Wyoming, station 06299980, (2) State line near Decker, Montana, station 06306300, (3) Tongue River Dam near Decker, Montana, station 06307500, (4) Birney Day School Bridge near Birney, Montana, station 06307616, (5) below Brandenberg Bridge near Ashland, Montana, station 06307830, (6) above T\&Y Diversion Dam near Miles City, Montana, station 06307990, and (7) Miles City, Montana, station 06308500. Water samples were collected and analyzed from five sites on tributaries located at: (1) Goose Creek near Acme, Wyoming, station 06305700, (2) Prairie Dog Creek near Acme, Wyoming, station 06306250, (3) Hanging Woman Creek near Birney, Montana, station 06307600, (4) Otter Creek at Ashland, Montana, station 06307740, and (5) Pumpkin Creek near Miles City, Montana, station 06308400. All water-quality data for samples collected at these 12 sites can be accessed at Web sites http://waterdata.usgs.gov/mt/nwis or http://waterdata.usgs.gov/wy/nwis.

\section{Introduction}

The northward-flowing Tongue River drains an area of about 5,400 square miles in the coal-rich areas of northeastern Wyoming and southeastern Montana. From its headwaters in the Bighorn National Forest of Wyoming, the Tongue River flows through rolling foothills, dissected and tree-studded uplands, and undulating grass plains, to join the Yellowstone River at Miles City, Montana (fig. 1). The river flows through lands historically used for livestock grazing and production of irrigated crops. About 86,000 acres of land in the Tongue River watershed are irrigated, with about 64,000 acres in Wyoming and 22,000 acres in Montana (Cannon and Johnson, 2004; Berkas and others, 2006). Irrigation of lands in the lower part of the basin was increased after construction of Tongue River Dam, which was completed in 1939 and retrofitted in 1999. Tongue River Reservoir currently (2007) can store about 79,000 acre-feet of water. Water from Tongue River Reservoir is used to irrigate more than 14,000 acres between the dam and Miles City, Montana.

Coal deposits in the upper part of the Tongue River watershed are extracted at large surface coal mines near Acme, Wyoming, and Decker, Montana. Since about 1999, coal-bed methane (CBM) resources underlying the Tongue River watershed near the border of Wyoming and Montana have been a major focus of energy exploration and development. Production of CBM requires the pumping of large volumes of ground 
water from the coal beds to reduce water pressure within the formation and release the stored gas. Water from the coal beds typically is high in sodium and low in calcium and magnesium (Van Voast, 2003), resulting in a high sodium-adsorption ratio (SAR). Disposal of ground water with high concentrations of sodium into the Tongue River has the potential to increase salinity and SAR of water in the river and potentially reduce the quality of water for irrigation purposes. Water-quality standards for specific conductance (SC) and SAR have been established for the Montana portion of the Tongue River watershed by the Montana Department of Environmental Quality (2003).

Starting in 2004, Congress appropriated funding for the U.S. Geological Survey (USGS) to initiate and operate a comprehensive surface-water-quality monitoring network in the
Tongue River watershed to describe current (2004-06) conditions and potential changes in streams. A monitoring network consisting of 12 water-quality stations was established by the USGS during 2004 and 2005 (fig. 1) with support from the Congressional funding and cooperative programs with several Federal, State, Tribal, and local entities. Seven monitoring stations were established on the main stem of the Tongue River to identify downstream changes in water quality. Five stations were established on major tributaries to the Tongue River to document water quality in these smaller watersheds. Continuous streamflow and SC data were collected at each station during the general irrigation season (March-October). Additionally, water samples were collected at the monitoring stations at regular intervals and analyzed for major ions, nutrients, trace elements, and suspended sediment.

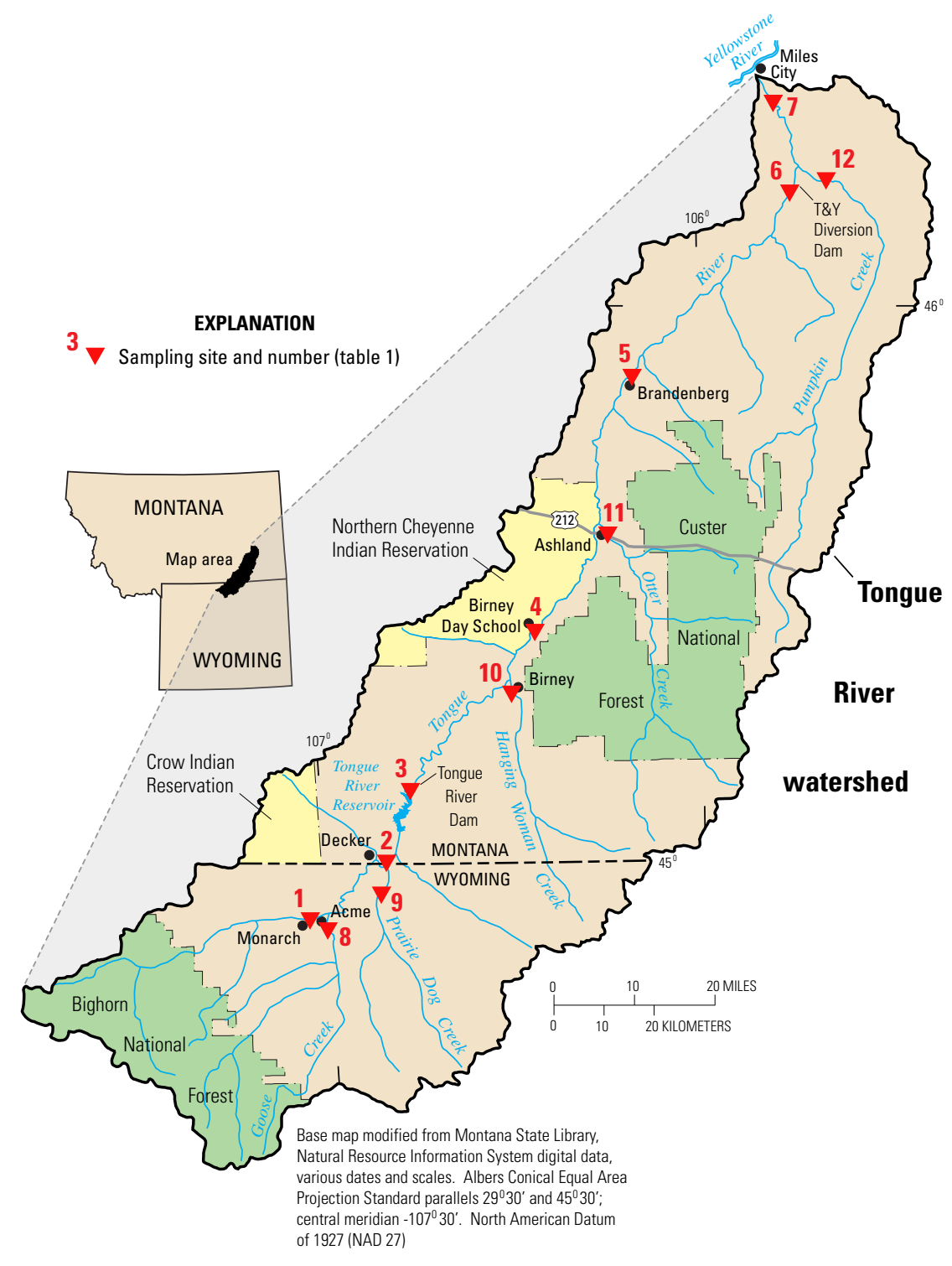

Figure 1. Tongue River watershed and location of sampling sites, Montana and Wyoming. 
Estimation and real-time reporting of SAR is desirable for management of irrigation systems, but SAR is difficult to determine in the field on a real-time basis. Direct determination of SAR typically requires laboratory analysis of water samples to determine concentrations of calcium, magnesium, and sodium, which are then used to calculate SAR. Such determinations can be relatively labor intensive and untimely because on-site water-sample collection is required and the results of sample analysis might not be available for many days. Therefore, an alternate method for estimating SAR in real-time from a strongly related, yet easily measured, surrogate was developed.

In natural waters of many areas, SAR is positively correlated with the dissolved-solids concentration; that is, SAR increases as dissolved-solids concentration increases. Factors that result in changes in dissolved-solids concentrations also influence the relative proportions of calcium, magnesium, and sodium. During periods of surface runoff of rainfall or snowmelt, the water has limited contact time with rock and soil materials. Consequently, runoff provides relatively dilute water to streams, which decreases dissolved-solids concentrations in the streamflow. As surface runoff is depleted and streamflow decreases, ground water contributes an increasingly greater proportion of water to streamflow. Ground water is commonly higher in dissolved-solids concentration relative to surface runoff as a result of long contact time with rock and soil materials. Therefore, dissolved-solids concentrations typically increase as streamflow decreases. Where ground water has high sodium concentrations relative to calcium and magnesium, SAR increases concurrently with increases in dissolved-solids concentrations. This relation enables estimation of SAR either directly from dissolved-solids concentration (a laboratory determination) or from a surrogate index of dissolved solids-concentration, such as SC (a field measurement).

$\mathrm{SC}$ is a standardized measurement of the electrical conductance of a solution that commonly is strongly related to dissolved-solids concentration. Electrical conductance is the ability of a solution to conduct an electric current and is dependent upon the dissolved-solids concentration, the ionic mobilities of the specific ions in the solution, and the temperature of the solution. SC can be measured in the field with continuously operating instrumentation. Because SAR also is related to dissolved-solids concentration, $\mathrm{SC}$ can serve as a useful surrogate for SAR by using linear regression methods to determine equations for estimating SAR based on SC. Thus, continuous measurement of SC coupled with statistically derived equations can provide a method for real-time reporting of estimated SAR.

Although SAR and SC might be related statistically, the existence of such a relation does not necessarily mean that fluctuations in SC cause fluctuations in SAR. Typically, causeand-effect interactions are more complex than the simple mathematical correlation between two variables. But, if the correlation is strong enough, linear regression becomes a useful tool for estimating water-quality conditions. In some cases, various factors can result in weak relations between SAR and SC. For example, in areas where there is substantial variability in the relative proportions of sodium, calcium, and magnesium in different tributary waters flowing to and mixing in a receiving stream, the relation between SAR and SC in the receiving stream might be weak. The mixing characteristics might be further complicated by variable timing of runoff contributed by the tributaries. In this study, the statistical relations between SAR and SC for the individual monitoring sites were evaluated by regression analysis for suitability of using continuous $\mathrm{SC}$ measurements for reporting real-time estimates of SAR.

\section{Purpose and Scope}

The purpose of this report is to document measured and estimated SAR for Tongue River and its tributaries in Montana and Wyoming. SC and SAR values were measured in water samples collected periodically at 12 monitoring sites in the Tongue River watershed (table 1). In addition, regression relations between SC and SAR were analyzed for each site for the years 2004-06 and appropriate regression equations were developed for estimating SAR from SC for most sites. The regression equations developed for monitoring sites in the Tongue River watershed that were statistically significant were used with the continuous SC data to estimate daily SAR during the 2004 and 2005 irrigation seasons (generally March-October). The 2005 equations also were used to estimate 2006 provisional SAR values, which were displayed on the Web in real-time.

\section{Study Methods}

\section{Methods of Data Collection}

Water samples typically were collected 12-18 times per year at each site. Sites on the main stem of the Tongue River were sampled more frequently than tributary sites. During 2004-05, sampling was more frequent during the irrigation season than during the non-irrigation season. Some tributaries were dry during some sampling visits, so fewer samples were collected at those sites.

Cross-sectional water samples were collected from multiple verticals across the stream using depth- and widthintegration methods described by Ward and Harr (1990), Edwards and Glysson (1999), and U.S. Geological Survey (variously dated). These methods provide a vertically and laterally discharge-weighted sample that is representative of the entire flow passing through the cross section of a stream. Grab samples were collected when streamflow was too low for depth integration. Sampling equipment consisted of standard USGS depth-integrating suspended-sediment samplers (DH-48, DH-81, D-74, and D-95). Samplers equipped with nylon nozzles and coated with a non-metallic epoxy paint or constructed of plastic were used to collect samples for analysis of trace elements. All samplers are designed to sample the 
Table 1. Site information and summary of the number of water-quality samples collected during various years by the U.S. Geological Survey at sites in the Tongue River surface-water-quality monitoring network.

[Shaded cells indicate the period of record and number of samples used to develop the various regression equations for estimating sodium-adsorption ratio from specific conductance (no data are available for any of the sites for 1997-98). No pre-1990 data were used to develop regression equations. Abbreviation: USGS, U.S. Geological Survey]

\begin{tabular}{|c|c|c|c|c|c|c|}
\hline $\begin{array}{c}\text { Site } \\
\text { number } \\
\text { (fig. 1) }\end{array}$ & $\begin{array}{c}\text { USGS } \\
\text { streamflow- } \\
\text { gaging } \\
\text { station } \\
\text { number }\end{array}$ & Site name & \multicolumn{4}{|c|}{ Number of samples } \\
\hline & & MAIN STEM SITES & & & & \\
\hline 1 & 06299980 & Tongue River at Monarch, Wyoming & 79 & 0 & 0 & 32 \\
\hline 3 & 06307500 & $\begin{array}{l}\text { Tongue River at Tongue River Dam, near Decker, } \\
\text { Montana }\end{array}$ & 134 & 51 & 0 & 36 \\
\hline 4 & 06307616 & $\begin{array}{l}\text { Tongue River at Birney Day School Bridge, near } \\
\text { Birney, Montana }\end{array}$ & 82 & 11 & 0 & 36 \\
\hline 5 & 06307830 & $\begin{array}{l}\text { Tongue River below Brandenberg Bridge, near Ash- } \\
\text { land, Montana }\end{array}$ & 71 & 0 & 43 & 35 \\
\hline \multirow[t]{2}{*}{7} & 06308500 & Tongue River at Miles City, Montana & 214 & 19 & 9 & 34 \\
\hline & & TRIBUTARY SITES & & & & \\
\hline 8 & 06305700 & Goose Creek near Acme, Wyoming & 0 & 0 & 0 & 23 \\
\hline 9 & 06306250 & Prairie Dog Creek near Acme, Wyoming & 0 & 0 & 41 & 27 \\
\hline 10 & 06307600 & Hanging Woman Creek near Birney, Montana & 122 & 23 & 5 & 19 \\
\hline 11 & 06307740 & Otter Creek at Ashland, Montana & 119 & 23 & 6 & 23 \\
\hline 12 & 06308400 & Pumpkin Creek near Miles City, Montana & 55 & 0 & 0 & 17 \\
\hline
\end{tabular}

water column isokinetically, whereby velocity flow paths are not distorted within the nozzle, thus allowing suspended particles to enter the sample-collection bottle at the same concentration as in the surrounding water.

Instantaneous streamflow at the time of sampling was determined at all sites, either by direct measurement or from stage-discharge rating tables (Rantz and others, 1982). At some sites, streamflow was estimated for some days because no direct measurement was made and the stage-discharge relation was indeterminate or gage-height record was missing. Methods used to estimate streamflow are described in detail by Rantz and others (1982). Estimated streamflow values were flagged in the data tables with an E.

Onsite measurements of $\mathrm{pH}$, specific conductance, and water temperature also were made during all sampling visits. Onsite sample processing - including withdrawal of representative subsamples from the composite cross-section sample, filtration of samples for dissolved constituents, and preservation of samples to prevent degradation prior to analysis- was performed according to procedures described by Ward and Harr (1990), Horowitz and others (1994), and U.S. Geological
Survey (variously dated). Selected sample types were stabilized for analysis, using either acid preservative or chilling.

Water samples were analyzed for chemical constituents by the U.S. Geological Survey National Water Quality Laboratory (NWQL) in Denver, Colorado. Dissolved concentrations of constituents were analyzed from filtered subsamples obtained by pumping the original sample water through a filter having a pore size of $0.45 \mu \mathrm{m}$ to remove particulate material. Total-recoverable concentrations of trace elements were determined from an unfiltered subsample that was digested in the laboratory with a solution of weak acid to liberate adsorbed and acid-soluble constituents from particulate material (Hoffman and others, 1996). Methods used by the NWQL to analyze samples are described by Fishman and Friedman (1989), Fishman (1993), McLain (1993), Garbarino and Struzeski (1998), and Jones and Garbarino (1999).

Cross-sectional water samples also were analyzed for suspended-sediment concentration and percent of suspended sediment finer than $0.062 \mathrm{~mm}$ in diameter (silt size and smaller) by the USGS Montana sediment laboratory in Helena, 
Montana. The methods for analysis of suspended sediment are described by Guy (1969) and Dodge and Lambing (2006).

A continuous record (15-minute recording interval) of specific conductance was obtained at selected sites by use of an electronic sensor that was placed in the stream where depth and velocity were adequate to generally represent the average specific conductance in the cross section. The conductance monitors were inspected on every visit to the site and checked for accuracy and drift according to methods described in Wagner and others (2006). Specific-conductance data were transmitted hourly by satellite telemetry and displayed in real-time on the USGS National Water Information System (NWIS) Web page for Montana (http://waterdata.usgs.gov/mt/nwis/). This same Web page also displays real-time streamflow data for USGS gaging stations.

Quality-assurance procedures used for the collection and field processing of water-quality samples are described by Ward and Harr (1990), Knapton and Nimick (1991), Horowitz and others (1994), Edwards and Glysson (1999), and USGS (variously dated). Standard procedures used by the NWQL for internal sample handling and quality assurance are described by Friedman and Erdmann (1982), Jones (1987), and Pritt and Raese (1995). Quality-assurance procedures used by the Montana sediment laboratory are described by Dodge and Lambing (2006). Sample bottles, filters, and preservatives underwent systematic quality-control checks to verify cleanliness of material adequate to support analysis of environmental samples at low concentrations (such as $\mu \mathrm{g} / \mathrm{L}$, also referred to as "parts per billion").

The quality of analytical results reported for water-quality samples was evaluated by quality-control samples that were submitted from the field and analyzed concurrently in the laboratory with routine samples. These quality-control samples consisted of replicates and blanks which provide quantitative information on the precision and bias of the overall field and laboratory process. The average relative percent difference (RPD) for calcium, magnesium, and sodium concentrations in the 14 field-replicate samples collected between October 2003 and October 2005 was 1.3 percent, 0.8 percent, and 1.3 percent, respectively. The maximum RPD was 3.1 percent, 2.4 percent, and 4.0 percent, respectively. RPD values were calculated by dividing the difference in concentration of the two replicates by the average of the two concentrations, and then multiplying the result by 100 . Concentrations of calcium, magnesium, and sodium were less than the minimum reporting level in the 12 field-blank samples collected during the same period.

\section{Methods of Data Analysis}

The SAR in surface water can be related to SC because both parameters are a function of the concentrations of major ions in water. Therefore, SAR potentially can be estimated from SC using statistical techniques. Ordinary least-squares regression (also known as linear regression) is a well known and commonly used estimation technique (Helsel and Hirsch, 1992) and was used for estimating SAR from SC at monitoring stations in the Tongue River watershed. Developing regression models (equations) for the monitoring stations in the Tongue River network required several steps. The first steps were to retrieve and compile existing data from the USGS NWIS database, and then develop criteria for selecting the data to use in the regression analyses. These criteria were the time period during which the samples were collected and the minimum number of samples needed to adequately characterize variability.

The next step in developing a regression model for a specific site was to make graphical plots of SC (independent variable) and SAR (dependent variable) and then examine patterns in the data. For one site (Tongue River at State line), transformation of SC and SAR values to logarithms produced a better regression model than using non-transformed values. For another site (Prairie Dog Creek near Acme), the relation between SC and SAR appeared to be different during the winter (November-March) than for the rest of the year. For this site, the winter data were not included in the regression analysis. Excluding winter data that display a distinctly different pattern was deemed acceptable because measurement of SC using continuous monitors was planned to be seasonal (March-October). However, if the SC-SAR relation for winter samples was essentially the same as the relation for the rest of the year, the winter data were used in development of the regression model. For Tongue River near Miles City, data for the June 9, 1993, and June 9, 2005, samples appeared to be outliers because the SAR values (3.43 and 3.71) were much higher than SAR values for other samples with similar SC. Both samples were collected during high flows generated by local storms and were not considered typical. Therefore, data for these samples were not used in the regression analyses.

Regression equations in this report are presented in the $y$-intercept slope form for a straight line in rectangular coordinates $(y=y$-intercept + slope $(x))$. SAR is the dependent variable and SC is the independent variable, resulting in a linear equation of the form (SAR $=y$-intercept + slope $(\mathrm{SC})$ ).

The strength of a statistical relation can be described mathematically using the correlation coefficient $(r)$, the coefficient of determination $\left(R^{2}\right)$, and the $p$-value. The correlation coefficient measures the strength of association between two variables, and the coefficient of determination indicates the portion of the variance in SAR that is explained by SC. The $p$-value is the probability that the slope of the regression equation is zero or horizontal, which would indicate the absence of a relation. Only regression equations with a $p$-value of less than 0.05 were considered statistically significant and used for estimating SAR.

Prediction intervals (dashed lines in figures of regression relations) were determined to evaluate the uncertainty of estimating SAR from SC using the linear-regression models (Helsel and Hirsch, 1992). Prediction intervals define a range of values for the dependent variable (SAR) for a given level of uncertainty. For each model, 95-percent prediction intervals 
were determined. For any given value of specific conductance, the 95-percent prediction interval represents the range of values within which the true value of SAR will occur 95 percent of the time. The larger the range of values (distance between prediction lines), the larger the uncertainty associated with the regression model.

\section{Measured and Estimated Sodium- Adsorption Ratio for Tongue River and its Tributaries}

Water samples from the 12 water-quality monitoring stations on Tongue River and its tributaries were collected under various flow conditions and at different times of the year in an attempt to obtain samples that reflect seasonal and annual changes in water quality. SAR in water samples was determined using equation 1 with laboratory-measured concentrations of sodium, calcium, and magnesium:

$$
S A R=\frac{N a^{+}}{\sqrt{\frac{C a^{2+}+M g^{2+}}{2}}}
$$

where

$$
\begin{aligned}
\mathrm{Na}^{+}, \mathrm{Ca}^{2+} \text {, and } \mathrm{Mg}^{2+} & \text { are sodium, calcium, and } \\
& \text { magnesium concentrations in } \\
& \text { milliequivalents per liter (Hem, 1985). }
\end{aligned}
$$

One objective of the USGS Tongue River surface-waterquality monitoring network is to report provisional values of estimated SAR in real-time on a continuous basis during the March-October irrigation season for sites on the Tongue River and its major tributaries. During 2004-06, this objective was accomplished for many of the sites by estimating provisional SAR values from the continuous seasonal record of provisional SC data provided by monitors installed at each network site. During the first 2 years of the monitoring program, some data collected prior to 2004 were used to develop regression equations because there were insufficient recent data. After the monitoring network had been in operation for 2 years, updated regression equations were developed using mostly data for years 2004-05, which represented a common time period for all sites.

Continuous SC data measured by monitors and SAR values estimated by regression equations that are displayed in real-time on the Web (http://waterdata.usgs.gov/) are provisional and subject to revision following various qualityassurance checks (Wagner and others, 2006). Consequently, the provisional SC and SAR data that were displayed in realtime on the Web may be different from the final record. After review, corrections, and approval, the SC data are considered final. Regression equations for estimating SAR are updated every year in order to incorporate the most recent sample data into the regression equations. These updated equations are then applied to the final SC data to produce a final record of estimated SAR.

The discrete SC measurements made by the continuous monitors every 15 minutes and displayed in real-time are referred to as unit values. The regression equations were applied to each SC unit value to generate a continuous record of provisional estimated SAR, which also was displayed in real-time. Daily mean estimated SAR then was calculated as the mean of all the individual estimates of SAR generated each day. The daily mean values of estimated SAR shown in the figures in the following sections represent the final values after applying updated equations to the final SC values. 


\section{Tongue River at Monarch, Wyoming}

This station (USGS station 06299980) is located in Sheridan County, Wyoming, 0.9 mile east of Monarch (fig. 1). The station is at an altitude of about 3,620 feet (NGVD 29) and has a drainage area of 478 square miles.

\section{Sodium-Adsorption Ratio Measured from Water Samples}

Thirty-two water samples were collected at this station from January 2004 through October 2005 (table 2). Samples were analyzed for major ions, nutrients, trace elements, and suspended sediment. Only values for instantaneous streamflow, SC, and SAR are shown; values for the remaining constituents can be accessed at http://waterdata.usgs.gov/wy/nwis.

\section{Estimated Sodium-Adsorption Ratio and Regression Equations for 2004-06}

SAR values for Tongue River at Monarch for the 2004 irrigation season were estimated using continuous SC data and a regression equation (fig. $2 A$ ) based on the SC and SAR values from 17 water samples collected in 2004 (table 2). SAR values for the 2005 irrigation season were estimated using continuous $\mathrm{SC}$ and a regression equation (fig. $2 B$ ) based on data from all 32 samples from 2004-05 (table 2). Provisional real-time SAR data for 2006 also were estimated using the equation for the 2005 irrigation season (fig. 2B). Comparisons of sample SAR to daily mean estimated SAR for the 2004 and 2005 irrigation seasons are shown on figure 3 . 
Table 2. Water-sample data collected at Tongue River at Monarch, Wyoming (06299980), and used in regression equations for estimating sodium-adsorption ratio from specific conductance.

[Abbreviations: $\mathrm{ft}^{3} / \mathrm{s}$, cubic feet per second; SC, specific conductance; $\mu \mathrm{S} / \mathrm{cm}$, microsiemens per centimeter at 25 degrees Celsius; SAR, sodium-adsorption ratio; E, estimated value for streamflow. Symbol: --, data not used]

\begin{tabular}{|c|c|c|c|c|c|c|}
\hline \multirow[b]{2}{*}{ Date } & \multirow[b]{2}{*}{$\begin{array}{l}\text { Streamflow, } \\
\text { in } \mathrm{ft}^{3} / \mathrm{s}\end{array}$} & \multirow[b]{2}{*}{$\begin{array}{c}\text { SC, } \\
\text { in } \mu S / c m\end{array}$} & \multirow[b]{2}{*}{ SAR } & \multicolumn{3}{|c|}{$\begin{array}{l}\text { Sample data used for } \\
\text { regression equation }\end{array}$} \\
\hline & & & & $\begin{array}{l}2004 \\
\text { final }\end{array}$ & $\begin{array}{l}2005 \\
\text { final }\end{array}$ & $\begin{array}{c}2006 \\
\text { provisional } \\
\text { real-time }\end{array}$ \\
\hline $01 / 20 / 2004$ & 62 & 414 & 0.27 & $\mathrm{X}$ & $\mathrm{X}$ & $\mathrm{X}$ \\
\hline $02 / 04 / 2004$ & 59 & 440 & .30 & $\mathrm{X}$ & $\mathrm{X}$ & $\mathrm{X}$ \\
\hline $02 / 24 / 2004$ & 66 & 432 & .32 & $\mathrm{X}$ & $\mathrm{X}$ & $\mathrm{X}$ \\
\hline 03/08/2004 & 64 & 421 & .39 & $\mathrm{X}$ & $\mathrm{X}$ & $\mathrm{X}$ \\
\hline 03/23/2004 & 67 & 456 & .42 & $\mathrm{X}$ & $\mathrm{X}$ & $\mathrm{X}$ \\
\hline $04 / 14 / 2004$ & 102 & 368 & .30 & $\mathrm{X}$ & $\mathrm{X}$ & $X$ \\
\hline $04 / 27 / 2004$ & 57 & 416 & .34 & $\mathrm{X}$ & $\mathrm{X}$ & $\mathrm{X}$ \\
\hline 05/13/2004 & 183 & 263 & .25 & $\mathrm{X}$ & $\mathrm{X}$ & $\mathrm{X}$ \\
\hline $05 / 26 / 2004$ & 174 & 316 & .27 & $\mathrm{X}$ & $\mathrm{X}$ & $\mathrm{X}$ \\
\hline 06/10/2004 & 147 & 297 & .27 & $\mathrm{X}$ & $\mathrm{X}$ & $\mathrm{X}$ \\
\hline $06 / 22 / 2004$ & 169 & 343 & .34 & $\mathrm{X}$ & $\mathrm{X}$ & $\mathrm{X}$ \\
\hline 07/14/2004 & 88 & 366 & .37 & $\mathrm{X}$ & $\mathrm{X}$ & $\mathrm{X}$ \\
\hline $07 / 27 / 2004$ & 56 & 393 & .40 & $\mathrm{X}$ & $\mathrm{X}$ & $\mathrm{X}$ \\
\hline 08/18/2004 & 41 & 535 & .51 & $\mathrm{X}$ & $\mathrm{X}$ & $\mathrm{X}$ \\
\hline 08/24/2004 & 30 & 499 & .53 & $\mathrm{X}$ & $\mathrm{X}$ & $\mathrm{X}$ \\
\hline 09/15/2004 & 41 & 503 & .52 & $\mathrm{X}$ & $\mathrm{X}$ & $\mathrm{X}$ \\
\hline 09/27/2004 & 71 & 396 & .33 & $\mathrm{X}$ & $\mathrm{X}$ & $\mathrm{X}$ \\
\hline $10 / 13 / 2004$ & 69 & 432 & .37 & -- & $\mathrm{X}$ & $\mathrm{X}$ \\
\hline $11 / 04 / 2004$ & 88 & 400 & .31 & -- & $\mathrm{X}$ & $\mathrm{X}$ \\
\hline $12 / 02 / 2004$ & E64 & 430 & .32 & -- & $\mathrm{X}$ & $\mathrm{X}$ \\
\hline 02/09/2005 & E60 & 495 & .39 & -- & $\mathrm{X}$ & $\mathrm{X}$ \\
\hline 03/09/2005 & 52 & 430 & .35 & -- & $\mathrm{X}$ & $\mathrm{X}$ \\
\hline 04/06/2005 & 60 & 406 & .32 & -- & $\mathrm{X}$ & $\mathrm{X}$ \\
\hline $04 / 20 / 2005$ & 161 & 286 & .22 & -- & $\mathrm{X}$ & $\mathrm{X}$ \\
\hline 05/03/2005 & 128 & 379 & .31 & -- & $\mathrm{X}$ & $\mathrm{X}$ \\
\hline 05/12/2005 & 1,150 & 347 & .47 & -- & $\mathrm{X}$ & $\mathrm{X}$ \\
\hline 06/09/2005 & 781 & 200 & .17 & -- & $\mathrm{X}$ & $\mathrm{X}$ \\
\hline $06 / 23 / 2005$ & 676 & 193 & .16 & -- & $\mathrm{X}$ & $\mathrm{X}$ \\
\hline $07 / 14 / 2005$ & 174 & 296 & .20 & -- & $\mathrm{X}$ & $\mathrm{X}$ \\
\hline 08/04/2005 & 112 & 377 & .28 & -- & $\mathrm{X}$ & $\mathrm{X}$ \\
\hline $08 / 25 / 2005$ & 96 & 414 & .34 & -- & $\mathrm{X}$ & $\mathrm{X}$ \\
\hline $10 / 19 / 2005$ & 92 & 401 & .30 & -- & $\mathrm{X}$ & $\mathrm{X}$ \\
\hline
\end{tabular}




\section{Tongue River at Monarch}

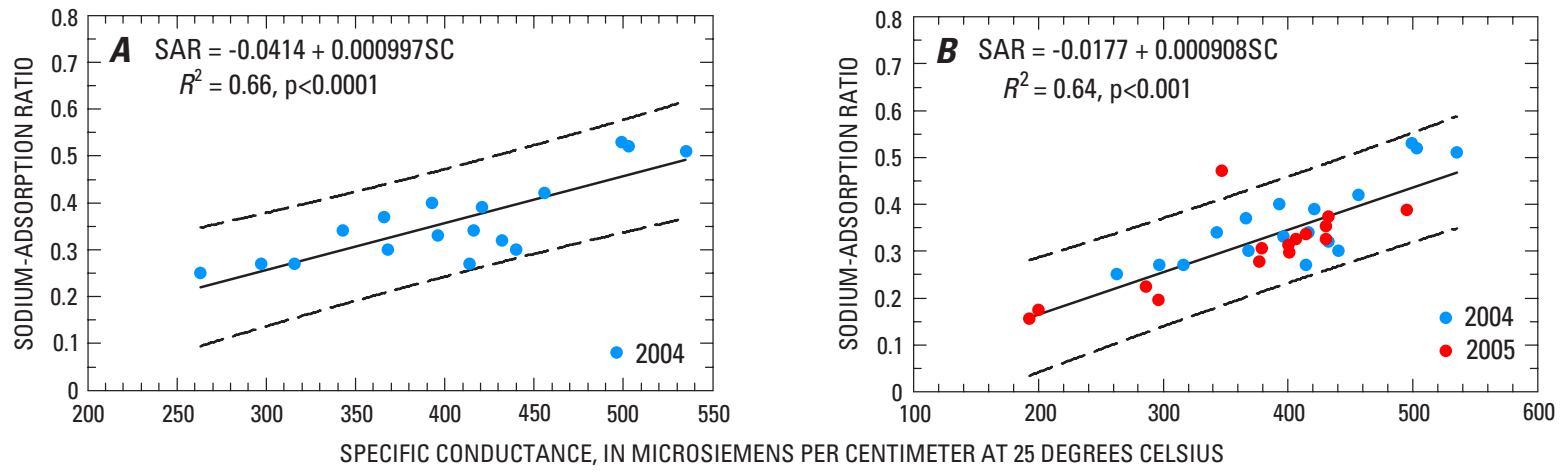

Figure 2. Relation of specific conductance (SC) and sodium-adsorption ratio (SAR) for water-quality samples collected at Tongue River at Monarch, Wyoming: $A$, Relation used to estimate SAR for the 2004 irrigation season and based on data collected January through September 2004; $B$, Relation used to estimate SAR for the 2005 irrigation season and based on data collected January 2004 through October 2005. Dashed lines indicate 95-percent prediction interval.

Tongue River at Monarch

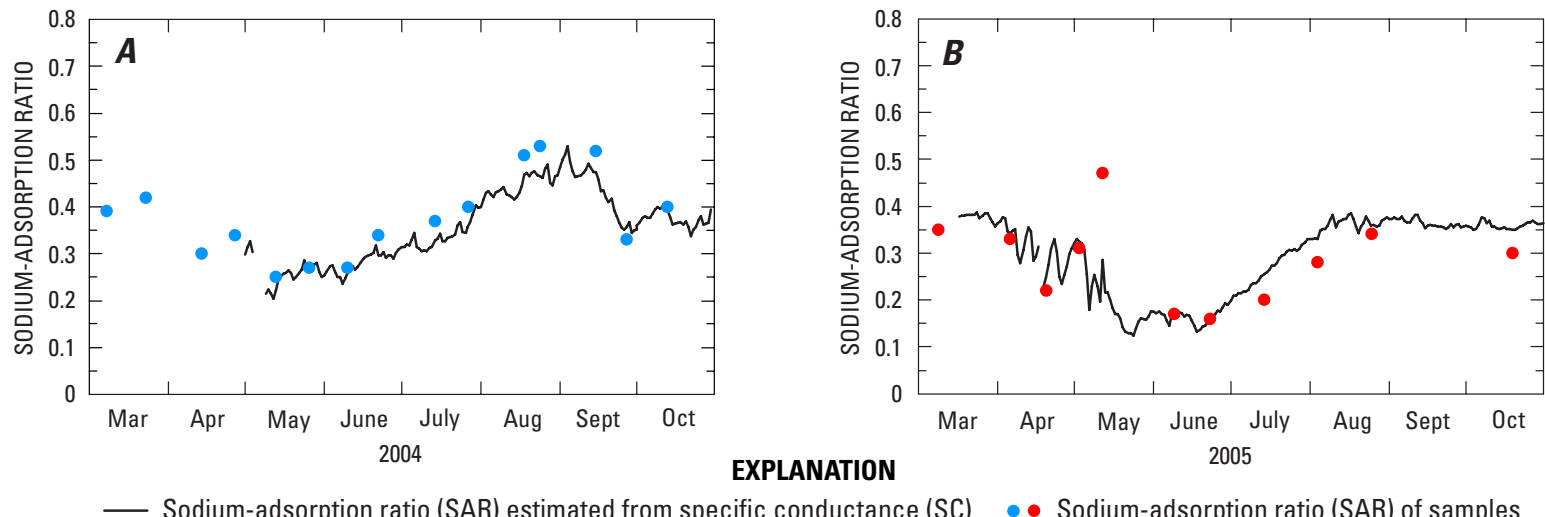

Figure 3. Sodium-adsorption ratio (SAR) determined from water-quality samples and estimated from specific conductance (SC) for Tongue River at Monarch, Wyoming, using relations shown in figure 2: $A$, 2004 irrigation season; $B, 2005$ irrigation season. 


\section{Tongue River at State line, near Decker, Montana}

This station (USGS station 06306300) is located in Big Horn County, Montana, 1 mile north of the WyomingMontana State line and 1.4 miles southeast of Decker (fig. 1). The station is at an altitude of 3,429 feet (NGVD 29) and has a drainage area of 1,453 square miles. This is the only site on the main stem of the Tongue River in Montana where streamflow is not regulated by the Tongue River Dam. Long-term mean monthly discharge for water years 1961-2005 ranged from 171 cubic feet per second in August to 1,595 cubic feet per second in June (Berkas and others, 2006).

\section{Sodium-Adsorption Ratio Measured from Water Samples}

Eighty-three water samples were collected at this station from October 1999 through October 2005 (table 3). Samples were analyzed for major ions, nutrients, trace elements, and suspended sediment. Only values for instantaneous streamflow, SC, and SAR are shown; values for the remaining constituents can be accessed at http://waterdata.usgs.gov/mt/nwis.

\section{Estimated Sodium-Adsorption Ratio and Regression Equations for 2004-06}

SAR values for Tongue River at State line for the 2004 irrigation season were estimated using continuous SC data and a regression equation (fig. $4 A$ ) based on the SC and SAR values from 62 water samples collected from October 1999 through September 2004 (table 3). SAR values for the 2005 irrigation season were estimated using continuous SC and a regression equation (fig. $4 B$ ) based on data from 39 water samples collected from November 2003 through October 2005 (table 3). Provisional real-time SAR data for 2006 also were estimated using the equation for the 2005 irrigation season (fig. 4B). Comparisons of sample SAR to daily mean estimated SAR for the 2004 and 2005 irrigation seasons are shown on figure 5.

Table 3. Water-sample data collected at Tongue River at State line, near Decker, Montana (06306300), and used in regression equations for estimating sodium-adsorption ratio from specific conductance.

[Abbreviations: $\mathrm{ft}^{3} / \mathrm{s}$, cubic feet per second; SC, specific conductance; $\mu \mathrm{S} / \mathrm{cm}$, microsiemens per centimeter at 25 degrees Celsius; SAR, sodium-adsorption ratio; E, estimated value for streamflow. Symbol: --, data not used]

\begin{tabular}{|c|c|c|c|c|c|c|}
\hline \multirow[b]{2}{*}{ Date } & \multirow{2}{*}{$\begin{array}{l}\text { Streamflow, } \\
\text { in } \mathrm{ft}^{3} / \mathrm{s}\end{array}$} & \multirow{2}{*}{$\begin{array}{c}S C, \\
\text { in } \mu S / c m\end{array}$} & \multirow[b]{2}{*}{ SAR } & \multicolumn{3}{|c|}{$\begin{array}{l}\text { Sample data used for } \\
\text { regression equation }\end{array}$} \\
\hline & & & & $\begin{array}{l}2004 \\
\text { final }\end{array}$ & $\begin{array}{l}2005 \\
\text { final }\end{array}$ & $\begin{array}{c}2006 \\
\text { provisional } \\
\text { real-time }\end{array}$ \\
\hline 10/14/1999 & 241 & 629 & 0.60 & $\mathrm{X}$ & -- & -- \\
\hline 01/05/2000 & 74 & 818 & .95 & $X$ & -- & -- \\
\hline 05/15/2000 & 519 & 348 & .41 & $X$ & -- & -- \\
\hline $06 / 21 / 2000$ & 725 & 362 & .40 & $X$ & -- & -- \\
\hline $07 / 25 / 2000$ & 146 & 587 & .65 & $X$ & -- & -- \\
\hline 08/02/2000 & 90 & 724 & .90 & $X$ & -- & -- \\
\hline $09 / 12 / 2000$ & 103 & 819 & .96 & $X$ & -- & -- \\
\hline $10 / 30 / 2000$ & 188 & 695 & .78 & $X$ & -- & -- \\
\hline $11 / 28 / 2000$ & E170 & 680 & .81 & $X$ & -- & -- \\
\hline $12 / 28 / 2000$ & E130 & 568 & .60 & $X$ & -- & -- \\
\hline 01/16/2001 & E185 & 586 & .72 & $X$ & -- & -- \\
\hline $02 / 12 / 2001$ & E180 & 622 & .69 & $X$ & -- & -- \\
\hline 03/12/2001 & E280 & 497 & .71 & $X$ & -- & -- \\
\hline $04 / 11 / 2001$ & 185 & 812 & .96 & $X$ & -- & -- \\
\hline 05/15/2001 & 420 & 256 & .34 & $X$ & -- & -- \\
\hline 06/19/2001 & 212 & 570 & .71 & $X$ & -- & -- \\
\hline $07 / 12 / 2001$ & 48 & 752 & 1.33 & $X$ & -- & -- \\
\hline
\end{tabular}


Table 3. Water-sample data collected at Tongue River at State line, near Decker, Montana (06306300), and used in regression equations for estimating sodium-adsorption ratio from specific conductance. - Continued

[Abbreviations: $\mathrm{ft}^{3} / \mathrm{s}$, cubic feet per second; SC, specific conductance; $\mu \mathrm{S} / \mathrm{cm}$, microsiemens per centimeter at 25 degrees Celsius; SAR, sodium-adsorption ratio; E, estimated value for streamflow. Symbol: --, data not used]

\begin{tabular}{|c|c|c|c|c|c|c|}
\hline \multirow[b]{2}{*}{ Date } & \multirow{2}{*}{$\begin{array}{l}\text { Streamflow, } \\
\text { in } \mathrm{ft}^{3} / \mathrm{s}\end{array}$} & \multirow{2}{*}{$\begin{array}{c}S C, \\
\text { in } \mu S / c m\end{array}$} & \multirow[b]{2}{*}{ SAR } & \multicolumn{3}{|c|}{$\begin{array}{l}\text { Sample data used for } \\
\text { regression equation }\end{array}$} \\
\hline & & & & $\begin{array}{l}2004 \\
\text { final }\end{array}$ & $\begin{array}{l}2005 \\
\text { final }\end{array}$ & $\begin{array}{c}2006 \\
\text { provisional } \\
\text { real-time }\end{array}$ \\
\hline $08 / 24 / 2001$ & 10 & 1,280 & 2.51 & $X$ & -- & -- \\
\hline 09/04/2001 & 18 & 1,280 & 2.76 & $X$ & -- & -- \\
\hline $10 / 01 / 2001$ & 66 & 870 & 1.28 & $X$ & -- & -- \\
\hline $11 / 15 / 2001$ & 131 & 765 & .98 & $X$ & -- & -- \\
\hline $12 / 11 / 2001$ & E100 & 760 & .88 & $X$ & -- & -- \\
\hline 01/07/2002 & E90 & 600 & .63 & $X$ & -- & -- \\
\hline $02 / 07 / 2002$ & E80 & 590 & .73 & $X$ & -- & -- \\
\hline 03/18/2002 & E90 & 583 & .78 & $X$ & -- & -- \\
\hline $04 / 03 / 2002$ & E90 & 699 & .89 & $X$ & -- & -- \\
\hline $05 / 29 / 2002$ & 314 & 330 & .45 & $X$ & -- & -- \\
\hline 06/05/2002 & 543 & 245 & .35 & $X$ & -- & -- \\
\hline $07 / 10 / 2002$ & 80 & 625 & 1.22 & $X$ & -- & -- \\
\hline 08/07/2002 & 50 & 990 & 1.99 & $X$ & -- & -- \\
\hline $09 / 16 / 2002$ & 114 & 735 & 1.00 & $X$ & -- & -- \\
\hline $10 / 01 / 2002$ & 134 & 694 & .86 & $\mathrm{X}$ & -- & -- \\
\hline $11 / 21 / 2002$ & 140 & 725 & .79 & $X$ & -- & -- \\
\hline $12 / 18 / 2002$ & E140 & 680 & .75 & $\mathrm{X}$ & -- & -- \\
\hline 01/07/2003 & E170 & 598 & .72 & $\mathrm{X}$ & -- & -- \\
\hline 02/06/2003 & 130 & 711 & .86 & $X$ & -- & -- \\
\hline 03/07/2003 & E170 & 751 & .85 & $\mathrm{X}$ & -- & -- \\
\hline $04 / 10 / 2003$ & 198 & 813 & .92 & $X$ & -- & -- \\
\hline 05/06/2003 & 480 & 488 & .61 & $\mathrm{X}$ & -- & -- \\
\hline 06/04/2003 & 1,740 & 222 & .29 & $\mathrm{X}$ & -- & -- \\
\hline 07/01/2003 & 769 & 282 & .36 & $X$ & -- & -- \\
\hline 08/05/2003 & 57 & 657 & 1.03 & $X$ & -- & -- \\
\hline 09/02/2003 & 106 & 796 & .87 & $X$ & -- & -- \\
\hline $10 / 06 / 2003$ & 162 & 643 & .74 & $X$ & -- & -- \\
\hline $11 / 18 / 2003$ & 198 & 636 & .69 & $\mathrm{X}$ & $\mathrm{X}$ & $\mathrm{X}$ \\
\hline $12 / 04 / 2003$ & E145 & 636 & .67 & $X$ & $X$ & $X$ \\
\hline $01 / 20 / 2004$ & E135 & 653 & .69 & $X$ & $X$ & $X$ \\
\hline $02 / 04 / 2004$ & E140 & 705 & .76 & $X$ & $X$ & $X$ \\
\hline $02 / 23 / 2004$ & E155 & 629 & .69 & $\mathrm{X}$ & $X$ & $X$ \\
\hline 03/09/2004 & E155 & 680 & .82 & $X$ & $X$ & $X$ \\
\hline $03 / 22 / 2004$ & 171 & 725 & .86 & $X$ & $X$ & $X$ \\
\hline $04 / 14 / 2004$ & 168 & 562 & .73 & $X$ & $X$ & $X$ \\
\hline $04 / 26 / 2004$ & 120 & 584 & .89 & $X$ & $X$ & $X$ \\
\hline
\end{tabular}


Table 3. Water-sample data collected at Tongue River at State line, near Decker, Montana (06306300), and used in regression equations for estimating sodium-adsorption ratio from specific conductance. - Continued

[Abbreviations: $\mathrm{ft}^{3} / \mathrm{s}$, cubic feet per second; $\mathrm{SC}$, specific conductance; $\mu \mathrm{S} / \mathrm{cm}$, microsiemens per centimeter at 25 degrees Celsius; SAR, sodium-adsorption ratio; E, estimated value for streamflow. Symbol: --, data not used]

\begin{tabular}{|c|c|c|c|c|c|c|}
\hline \multirow[b]{2}{*}{ Date } & \multirow[b]{2}{*}{$\begin{array}{l}\text { Streamflow, } \\
\text { in } \mathrm{ft}^{3} / \mathrm{s}\end{array}$} & \multirow[b]{2}{*}{$\begin{array}{c}S C, \\
\text { in } \mu \mathrm{S} / \mathrm{cm}\end{array}$} & \multirow[b]{2}{*}{ SAR } & \multicolumn{3}{|c|}{$\begin{array}{l}\text { Sample data used for } \\
\text { regression equation }\end{array}$} \\
\hline & & & & $\begin{array}{l}2004 \\
\text { final }\end{array}$ & $\begin{array}{l}2005 \\
\text { final }\end{array}$ & $\begin{array}{c}2006 \\
\text { provisional } \\
\text { real-time }\end{array}$ \\
\hline $05 / 13 / 2004$ & 237 & 400 & .62 & $\mathrm{X}$ & $X$ & $\mathrm{X}$ \\
\hline $05 / 26 / 2004$ & 219 & 477 & .67 & $\mathrm{X}$ & $X$ & $\mathrm{X}$ \\
\hline 06/09/2004 & 226 & 403 & .60 & $\mathrm{X}$ & $X$ & $\mathrm{X}$ \\
\hline $07 / 14 / 2004$ & 120 & 570 & .85 & $\mathrm{X}$ & $\mathrm{X}$ & $\mathrm{X}$ \\
\hline $07 / 27 / 2004$ & 106 & 634 & .83 & $\mathrm{X}$ & $X$ & $\mathrm{X}$ \\
\hline 08/18/2004 & 52 & 810 & 1.18 & $\mathrm{X}$ & $X$ & $\mathrm{X}$ \\
\hline $08 / 24 / 2004$ & 57 & 876 & 1.35 & $\mathrm{X}$ & $X$ & $\mathrm{X}$ \\
\hline $09 / 15 / 2004$ & 68 & 780 & 1.13 & $\mathrm{X}$ & $\mathrm{X}$ & $\mathrm{X}$ \\
\hline 09/27/2004 & 152 & 643 & .74 & $\mathrm{X}$ & $\mathrm{X}$ & $\mathrm{X}$ \\
\hline $10 / 13 / 2004$ & 137 & 707 & .78 & -- & $X$ & $\mathrm{X}$ \\
\hline $11 / 04 / 2004$ & 159 & 690 & .74 & -- & $X$ & $\mathrm{X}$ \\
\hline $12 / 02 / 2004$ & E150 & 775 & .82 & -- & $\mathrm{X}$ & $\mathrm{X}$ \\
\hline 02/09/2005 & E65 & 792 & .96 & -- & $X$ & $\mathrm{X}$ \\
\hline 03/09/2005 & 128 & 735 & .92 & -- & $\mathrm{X}$ & $\mathrm{X}$ \\
\hline $03 / 21 / 2005$ & 125 & 757 & .89 & -- & $\mathrm{X}$ & $\mathrm{X}$ \\
\hline $04 / 06 / 2005$ & 122 & 661 & .96 & -- & $\mathrm{X}$ & $\mathrm{X}$ \\
\hline $04 / 20 / 2005$ & 181 & 455 & .54 & -- & $\mathrm{X}$ & $\mathrm{X}$ \\
\hline 05/03/2005 & 149 & 633 & .81 & -- & $\mathrm{X}$ & $\mathrm{X}$ \\
\hline 05/12/2005 & 5,430 & 406 & .59 & -- & $\mathrm{X}$ & $\mathrm{X}$ \\
\hline 06/08/2005 & 1,730 & 236 & .33 & -- & $\mathrm{X}$ & $\mathrm{X}$ \\
\hline $06 / 22 / 2005$ & $\mathrm{E} 1,900$ & 186 & .27 & -- & $\mathrm{X}$ & $\mathrm{X}$ \\
\hline $07 / 14 / 2005$ & 306 & 465 & .59 & -- & $\mathrm{X}$ & $X$ \\
\hline $07 / 27 / 2005$ & 185 & 553 & .76 & -- & $\mathrm{X}$ & $\mathrm{X}$ \\
\hline 08/10/2005 & 125 & 659 & .84 & -- & $\mathrm{X}$ & $\mathrm{X}$ \\
\hline $08 / 24 / 2005$ & 181 & 629 & .81 & -- & $X$ & $\mathrm{X}$ \\
\hline 09/08/2005 & 137 & 675 & .80 & -- & $X$ & $\mathrm{X}$ \\
\hline 09/19/2005 & 162 & 634 & .72 & -- & $X$ & $X$ \\
\hline $09 / 26 / 2005$ & 198 & 623 & .67 & -- & $\mathrm{X}$ & $\mathrm{X}$ \\
\hline 09/27/2005 & 195 & 629 & .71 & -- & $\mathrm{X}$ & $\mathrm{X}$ \\
\hline $10 / 18 / 2005$ & 216 & 636 & .67 & -- & $X$ & $\mathrm{X}$ \\
\hline
\end{tabular}




\section{Tongue River at State line}
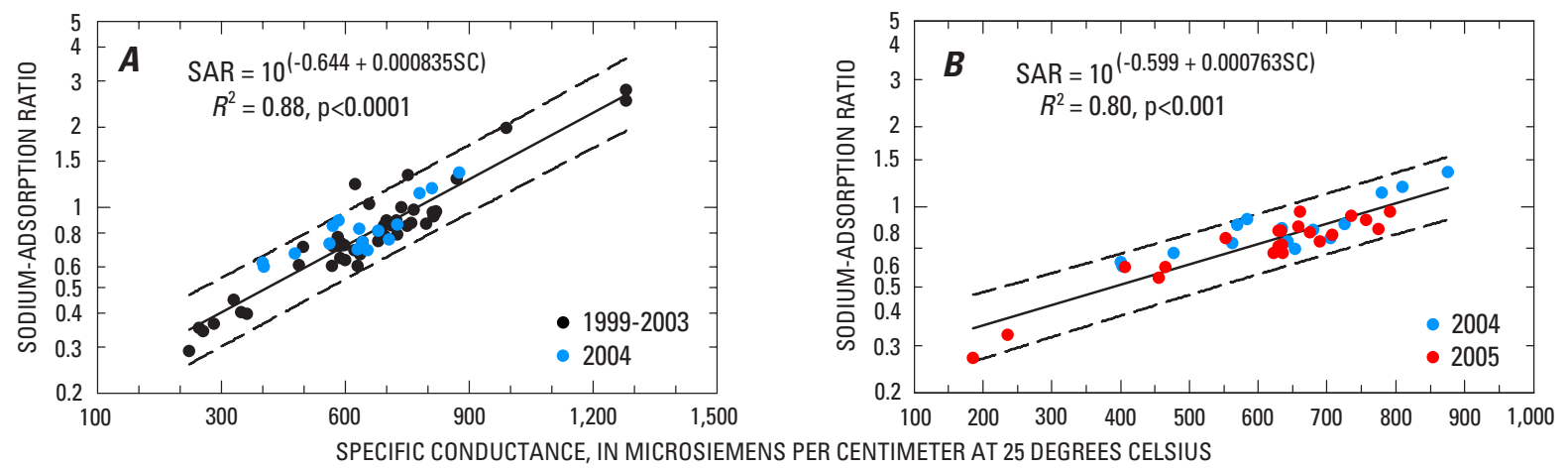

Figure 4. Relation of specific conductance (SC) and sodium-adsorption ratio (SAR) for water-quality samples collected at Tongue River at State line, near Decker, Montana: $A$, Relation used to estimate SAR for the 2004 irrigation season and based on data collected October 1999 through September 2004; $B$, Relation used to estimate SAR for the 2005 irrigation season and based on data collected November 2003 through October 2005. Dashed lines indicate 95-percent prediction interval.

Tongue River at State line
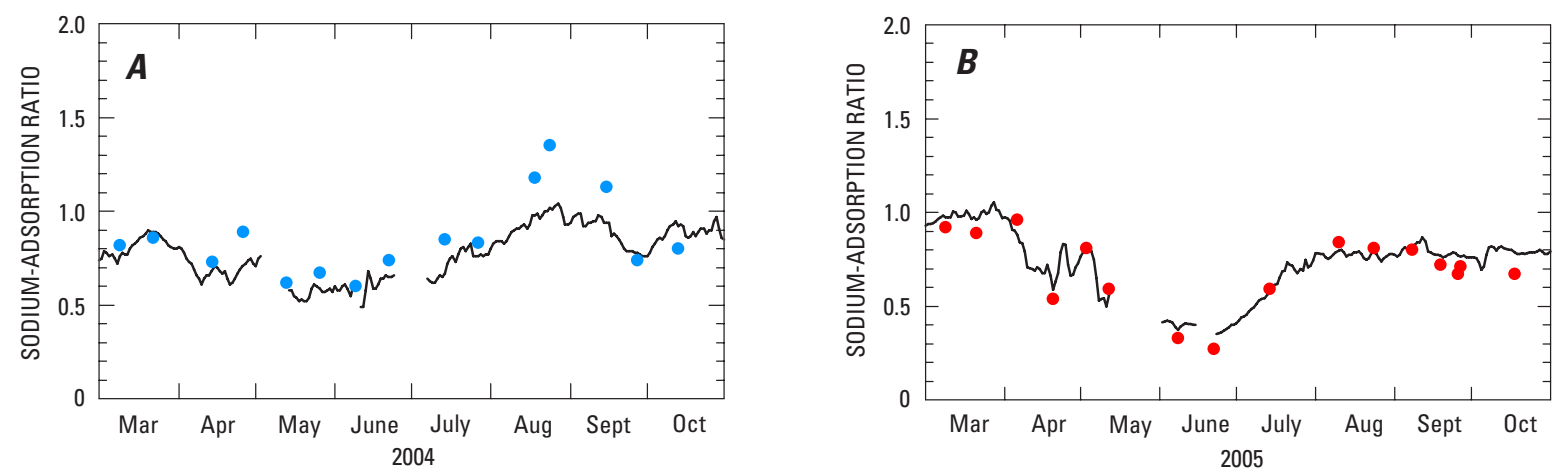

EXPLANATION

— Sodium-adsorption ratio (SAR) estimated from specific conductance (SC) • Sodium-adsorption ratio (SAR) of samples

Figure 5. Sodium-adsorption ratio (SAR) determined from water-quality samples and estimated from specific conductance (SC) for Tongue River at State line, near Decker, Montana, using relations shown in figure 4: A, 2004 irrigation season; $B, 2005$ irrigation season. 


\section{Tongue River at Tongue River Dam, near Decker, Montana}

This station (USGS station 06307500) is located in Big Horn County, Montana, 0.5 mile downstream from Tongue River Dam and 8 miles northeast of Decker (fig. 1). The station is at an altitude of 3,344 feet (NGVD 29) and has a drainage area of 1,770 square miles.

\section{Sodium-Adsorption Ratio Measured from Water Samples}

Thirty-six water samples were collected at this station from January 2004 through October 2005 (table 4). Samples were analyzed for major ions, nutrients, trace elements, and suspended sediment. Only values for instantaneous streamflow, SC, and SAR are shown; values for the remaining constituents can be accessed at http://waterdata.usgs.gov/mt/nwis.

\section{Estimated Sodium-Adsorption Ratio and Regression Equations for 2005-06}

SAR values for Tongue River at Tongue River Dam for the 2004 irrigation season were not estimated using continuous SC data because the regression relation based on the SC and SAR values from 17 water samples collected from January through September 2004 was not statistically significant. SAR values for the 2005 irrigation season were estimated using continuous SC and a regression equation (fig. 6) based on data from all 36 samples from January 2004 through October 2005 (table 4). Provisional real-time SAR data for 2006 also were estimated using this regression equation. A comparison of sample SAR to daily mean estimated SAR for the 2005 irrigation season is shown on figure 7. 
Table 4. Water-sample data collected at Tongue River at Tongue River Dam, near Decker, Montana (06307500), and used in regression equations for estimating sodium-adsorption ratio from specific conductance.

[Abbreviations: $\mathrm{ft}^{3} / \mathrm{s}$, cubic feet per second; SC, specific conductance; $\mu \mathrm{S} / \mathrm{cm}$, microsiemens per centimeter at 25 degrees Celsius; SAR, sodium-adsorption ratio; E, estimated value for streamflow. Symbol: --, data not used]

\begin{tabular}{|c|c|c|c|c|c|c|}
\hline \multirow[b]{2}{*}{ Date } & \multirow[b]{2}{*}{$\begin{array}{l}\text { Streamflow, } \\
\text { in } \mathrm{ft}^{3} / \mathrm{s}\end{array}$} & \multirow[b]{2}{*}{$\begin{array}{c}\text { SC, } \\
\text { in } \mu \mathrm{S} / \mathrm{cm}\end{array}$} & \multirow[b]{2}{*}{ SAR } & \multicolumn{3}{|c|}{$\begin{array}{l}\text { Sample data used for } \\
\text { regression equation }\end{array}$} \\
\hline & & & & $\begin{array}{c}2004 \\
\text { final' }\end{array}$ & $\begin{array}{l}2005 \\
\text { final }\end{array}$ & $\begin{array}{c}2006 \\
\text { provisional } \\
\text { real-time }\end{array}$ \\
\hline $01 / 20 / 2004$ & 120 & 713 & 0.84 & -- & $\mathrm{X}$ & $\mathrm{X}$ \\
\hline $02 / 04 / 2004$ & 118 & 705 & .91 & -- & $\mathrm{X}$ & $\mathrm{X}$ \\
\hline $02 / 23 / 2004$ & 114 & 715 & .81 & -- & $\mathrm{X}$ & $\mathrm{X}$ \\
\hline 03/09/2004 & 112 & 700 & .90 & -- & $\mathrm{X}$ & $\mathrm{X}$ \\
\hline $03 / 22 / 3004$ & 175 & 676 & .89 & -- & $\mathrm{X}$ & $\mathrm{X}$ \\
\hline $04 / 13 / 2004$ & 153 & 766 & .90 & -- & X & $\mathrm{X}$ \\
\hline $04 / 26 / 2004$ & 156 & 653 & .86 & -- & $\mathrm{X}$ & $\mathrm{X}$ \\
\hline $05 / 12 / 2004$ & 255 & 650 & .86 & -- & $\mathrm{X}$ & $\mathrm{X}$ \\
\hline $05 / 26 / 2004$ & 240 & 650 & .91 & -- & $\mathrm{X}$ & $\mathrm{X}$ \\
\hline 06/09/2004 & 198 & 646 & .92 & -- & $\mathrm{X}$ & $\mathrm{X}$ \\
\hline $06 / 22 / 2004$ & 210 & 634 & .92 & -- & $\mathrm{X}$ & $\mathrm{X}$ \\
\hline $07 / 13 / 2004$ & E255 & 629 & .94 & -- & $\mathrm{X}$ & $\mathrm{X}$ \\
\hline $07 / 27 / 2004$ & E290 & 607 & .86 & -- & $\mathrm{X}$ & $\mathrm{X}$ \\
\hline 08/17/2004 & E220 & 598 & .90 & -- & $\mathrm{X}$ & $\mathrm{X}$ \\
\hline $08 / 23 / 2004$ & E240 & 593 & .93 & -- & $\mathrm{X}$ & $\mathrm{X}$ \\
\hline $09 / 14 / 2004$ & E115 & 665 & 1.05 & -- & $\mathrm{X}$ & $\mathrm{X}$ \\
\hline $09 / 27 / 2004$ & 92 & 693 & 1.18 & -- & $\mathrm{X}$ & $\mathrm{X}$ \\
\hline $10 / 13 / 2004$ & 88 & 683 & 1.14 & -- & $\mathrm{X}$ & $\mathrm{X}$ \\
\hline $11 / 04 / 2004$ & 96 & 690 & 1.10 & -- & $\mathrm{X}$ & $\mathrm{X}$ \\
\hline $12 / 02 / 2004$ & 94 & 696 & 1.08 & -- & $\mathrm{X}$ & $\mathrm{X}$ \\
\hline 02/08/2005 & 75 & 735 & 1.04 & -- & $\mathrm{X}$ & $\mathrm{X}$ \\
\hline 03/08/2005 & 70 & 753 & 1.08 & -- & $\mathrm{X}$ & $\mathrm{X}$ \\
\hline $03 / 21 / 2005$ & 70 & 750 & 1.08 & -- & $\mathrm{X}$ & $\mathrm{X}$ \\
\hline $04 / 05 / 2005$ & 70 & 750 & 1.07 & -- & $\mathrm{X}$ & $\mathrm{X}$ \\
\hline $04 / 19 / 2005$ & 75 & 736 & 1.05 & -- & $\mathrm{X}$ & $\mathrm{X}$ \\
\hline $05 / 02 / 2005$ & 90 & 734 & 1.04 & -- & $\mathrm{X}$ & $\mathrm{X}$ \\
\hline $05 / 13 / 2005$ & 1,400 & 718 & 1.05 & -- & $\mathrm{X}$ & $\mathrm{X}$ \\
\hline $06 / 08 / 2005$ & 1,390 & 362 & .52 & -- & $\mathrm{X}$ & $\mathrm{X}$ \\
\hline $06 / 22 / 2005$ & 2,090 & 303 & .43 & -- & $\mathrm{X}$ & $\mathrm{X}$ \\
\hline $07 / 13 / 2005$ & 441 & 282 & .36 & -- & $\mathrm{X}$ & $\mathrm{X}$ \\
\hline $07 / 26 / 2005$ & 391 & 289 & .39 & -- & $\mathrm{X}$ & $\mathrm{X}$ \\
\hline 08/09/2005 & 420 & 342 & .44 & -- & $\mathrm{X}$ & $\mathrm{X}$ \\
\hline $08 / 24 / 2005$ & 367 & 433 & .60 & -- & $\mathrm{X}$ & $\mathrm{X}$ \\
\hline 09/07/2005 & 345 & 419 & .56 & -- & $\mathrm{X}$ & $\mathrm{X}$ \\
\hline 09/19/2005 & 220 & 459 & .58 & -- & $\mathrm{X}$ & $\mathrm{X}$ \\
\hline $10 / 18 / 2005$ & 213 & 560 & .74 & -- & $\mathrm{X}$ & $\mathrm{X}$ \\
\hline
\end{tabular}

${ }^{1}$ Regression relation is inadequate for estimation of SAR. 


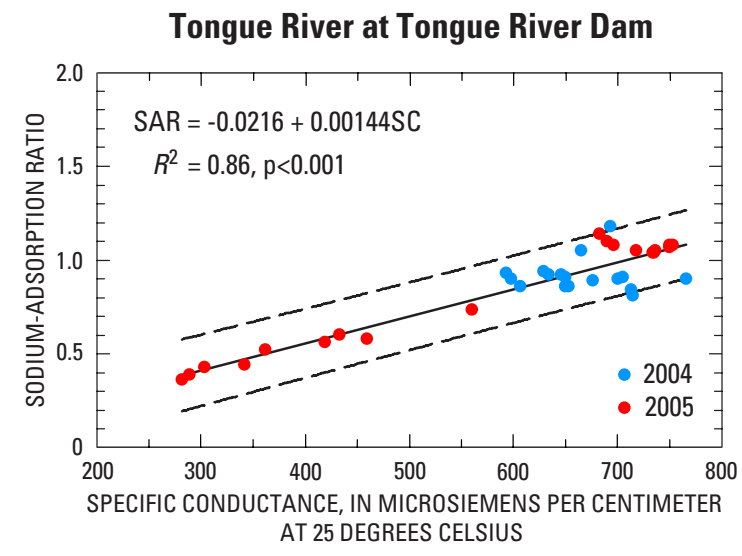

Figure 6. Relation of specific conductance (SC) and sodium-adsorption ratio (SAR) for water-quality samples collected January 2004 through October 2005 at Tongue River at Tongue River Dam, near Decker, Montana, and used to estimate SAR for the 2005 irrigation season. Dashed lines indicate 95-percent prediction interval.

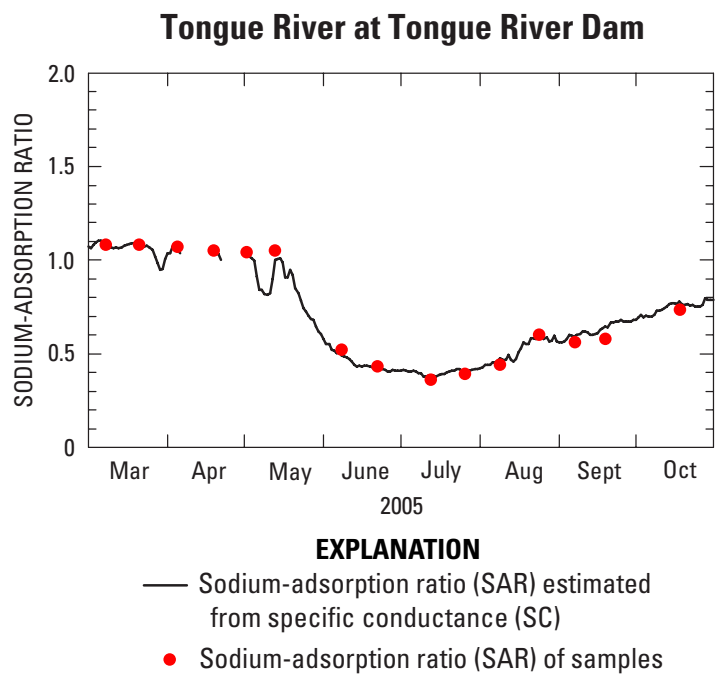

Figure 7. Sodium-adsorption ratio (SAR) determined from water-quality samples and estimated from specific conductance (SC) for Tongue River at Tongue River Dam, near Decker, Montana, for the 2005 irrigation season using relation shown in figure 6 . 


\section{Tongue River at Birney Day School Bridge, near Birney, Montana}

This station (USGS station 06307616) is located in Rosebud County, Montana, 0.2 mile east of Birney Day School (fig. 1). The station is at an altitude of 3,060 feet (NGVD 29) and has a drainage area of 2,621 square miles.

\section{Sodium-Adsorption Ratio Measured from Water Samples}

Thirty-six water samples were collected at this station from January 2004 through October 2005 (table 5). Samples were analyzed for major ions, nutrients, trace elements, and suspended sediment. Only values for instantaneous streamflow, SC, and SAR are shown; values for the remaining constituents can be accessed at http://waterdata.usgs.gov/mt/nwis.

\section{Estimated Sodium-Adsorption Ratio and Regression Equations for 2005-06}

SAR values for Tongue River at Birney Day School Bridge for the 2004 irrigation season were not estimated using continuous SC data because the regression relation based on the SC and SAR values from 17 water samples collected from January through September 2004 was not statistically significant. SAR values for the 2005 irrigation season were estimated using continuous SC and a regression equation (fig. 8) based on data from all 36 samples from January 2004 through October 2005 (table 5). Provisional real-time SAR data for 2006 also were estimated using this regression equation. A comparison of sample SAR to daily mean estimated SAR for the 2005 irrigation season is shown on figure 9 . 
Table 5. Water-sample data collected at Tongue River at Birney Day School Bridge, near Birney, Montana (06307616), and used in regression equations for estimating sodium-adsorption ratio from specific conductance.

[Abbreviations: $\mathrm{ft}^{3} / \mathrm{s}$, cubic feet per second; SC, specific conductance; $\mu \mathrm{S} / \mathrm{cm}$, microsiemens per centimeter at 25 degrees Celsius; SAR, sodium-adsorption ratio; E, estimated value for streamflow. Symbol: --, data not used]

\begin{tabular}{|c|c|c|c|c|c|c|}
\hline \multirow[b]{2}{*}{ Date } & \multirow{2}{*}{$\begin{array}{l}\text { Streamflow, } \\
\text { in } \mathrm{ft}^{3} / \mathrm{s}\end{array}$} & \multirow{2}{*}{$\begin{array}{c}S C, \\
\text { in } \mu S / c m\end{array}$} & \multirow[b]{2}{*}{ SAR } & \multicolumn{3}{|c|}{$\begin{array}{l}\text { Sample data used for } \\
\text { regression equation }\end{array}$} \\
\hline & & & & $\begin{array}{c}2004 \\
\text { final }^{1}\end{array}$ & $\begin{array}{l}2005 \\
\text { final }\end{array}$ & $\begin{array}{c}2006 \\
\text { provisional } \\
\text { real-time }\end{array}$ \\
\hline 01/07/2004 & E85 & 787 & 0.98 & -- & $\mathrm{X}$ & $\mathrm{X}$ \\
\hline 02/05/2004 & E95 & 762 & 1.02 & -- & $\mathrm{X}$ & $\mathrm{X}$ \\
\hline 02/23/2004 & E90 & 694 & .87 & -- & $\mathrm{X}$ & $\mathrm{X}$ \\
\hline 03/10/2004 & E135 & 680 & 1.04 & -- & $\mathrm{X}$ & $\mathrm{X}$ \\
\hline $03 / 22 / 3004$ & E181 & 703 & .97 & -- & $X$ & $X$ \\
\hline 04/13/2004 & 165 & 788 & 1.01 & -- & $\mathrm{X}$ & $\mathrm{X}$ \\
\hline $04 / 26 / 2004$ & 165 & 676 & 1.00 & -- & $\mathrm{X}$ & $\mathrm{X}$ \\
\hline 05/12/2004 & 236 & 664 & .93 & -- & $\mathrm{X}$ & $\mathrm{X}$ \\
\hline $05 / 25 / 2004$ & 218 & 671 & .98 & -- & $\mathrm{X}$ & $\mathrm{X}$ \\
\hline 06/08/2004 & 174 & 638 & 1.08 & -- & $\mathrm{X}$ & $\mathrm{X}$ \\
\hline $06 / 23 / 2004$ & 215 & 621 & 1.08 & -- & $\mathrm{X}$ & $\mathrm{X}$ \\
\hline 07/13/2004 & 220 & 630 & 1.04 & -- & $X$ & $X$ \\
\hline $07 / 26 / 2004$ & 288 & 604 & .94 & -- & $\mathrm{X}$ & $\mathrm{X}$ \\
\hline 08/17/2004 & 195 & 581 & .98 & -- & $\mathrm{X}$ & $\mathrm{X}$ \\
\hline $08 / 23 / 2004$ & 241 & 609 & 1.04 & -- & $X$ & $X$ \\
\hline 09/14/2004 & 109 & 624 & 1.19 & -- & $\mathrm{X}$ & $\mathrm{X}$ \\
\hline 09/27/2004 & 96 & 670 & 1.30 & -- & $\mathrm{X}$ & $\mathrm{X}$ \\
\hline $10 / 13 / 2004$ & 96 & 692 & 1.26 & -- & $X$ & $\mathrm{X}$ \\
\hline $11 / 04 / 2004$ & 96 & 720 & 1.23 & -- & $\mathrm{X}$ & $\mathrm{X}$ \\
\hline 12/08/2004 & E90 & 807 & 1.24 & -- & $\mathrm{X}$ & $\mathrm{X}$ \\
\hline 02/08/2005 & E70 & 805 & 1.20 & -- & $X$ & $X$ \\
\hline 03/08/2005 & 79 & 790 & 1.23 & -- & $\mathrm{X}$ & $\mathrm{X}$ \\
\hline 03/21/2005 & 83 & 770 & 1.18 & -- & $\mathrm{X}$ & $\mathrm{X}$ \\
\hline $04 / 05 / 2005$ & 75 & 773 & 1.27 & -- & $\mathrm{X}$ & $X$ \\
\hline 04/19/2005 & 75 & 760 & 1.27 & -- & $X$ & $X$ \\
\hline 05/02/2005 & 87 & 748 & .66 & -- & $\mathrm{X}$ & $\mathrm{X}$ \\
\hline 05/16/2005 & 1,320 & 730 & 1.06 & -- & $X$ & $X$ \\
\hline 06/08/2005 & 1,080 & 404 & .55 & -- & $\mathrm{X}$ & $\mathrm{X}$ \\
\hline $06 / 21 / 2005$ & 2,180 & 319 & .45 & -- & $\mathrm{X}$ & $\mathrm{X}$ \\
\hline 07/13/2005 & 509 & 325 & .46 & -- & $\mathrm{X}$ & $\mathrm{X}$ \\
\hline $07 / 26 / 2005$ & 457 & 390 & .43 & -- & $\mathrm{X}$ & $\mathrm{X}$ \\
\hline 08/09/2005 & 437 & 360 & .47 & -- & $\mathrm{X}$ & $\mathrm{X}$ \\
\hline $08 / 24 / 2005$ & 366 & 437 & .63 & -- & $\mathrm{X}$ & $\mathrm{X}$ \\
\hline 09/07/2005 & 319 & 470 & .65 & -- & $\mathrm{X}$ & $\mathrm{X}$ \\
\hline 09/19/2005 & 236 & 484 & .64 & -- & X & X \\
\hline $10 / 18 / 2005$ & 200 & 545 & .81 & -- & $\mathrm{X}$ & $X$ \\
\hline
\end{tabular}

${ }^{1}$ Regression relation is inadequate for estimation of SAR. 


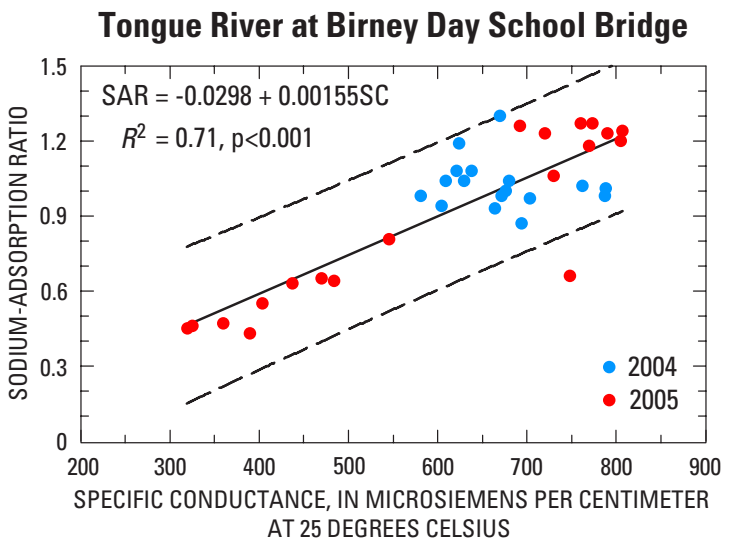

Figure 8. Relation of specific conductance (SC) and sodium-adsorption ratio (SAR) for water-quality samples collected January 2004 through October 2005 at Tongue River at Birney Day School Bridge, near Birney, Montana, and used to estimate SAR for the 2005 irrigation season. Dashed lines indicate 95-percent prediction interval.

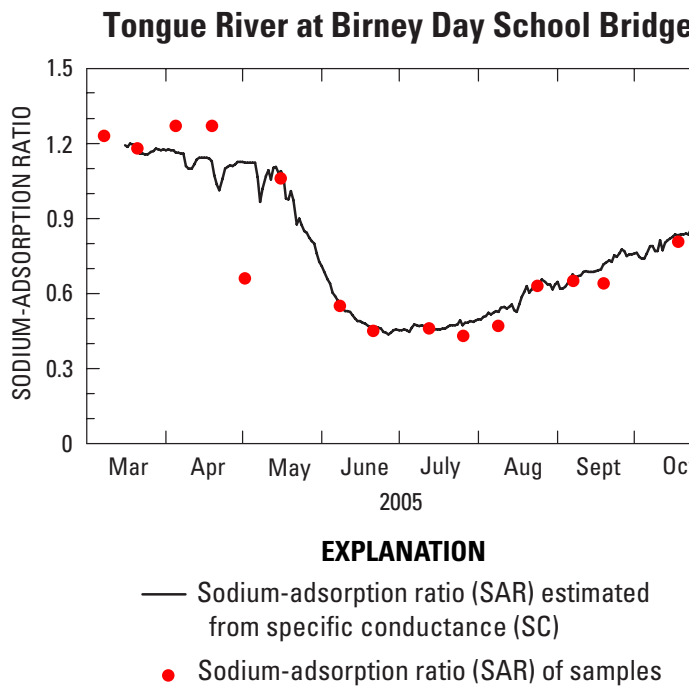

Figure 9. Sodium-adsorption ratio (SAR) determined from water-quality samples and estimated from specific conductance (SC) for Tongue River at Birney Day School Bridge, near Birney, Montana, for the 2005 irrigation season using relation shown in figure 8. 


\section{Tongue River below Brandenberg Bridge, near Ashland, Montana}

This station (USGS station 06307830) is located in Rosebud County, Montana, about 22 miles north of Ashland (fig. 1). The station is at an altitude of 2,760 feet (NGVD 29) and has a drainage area of 3,948 square miles.

\section{Sodium-Adsorption Ratio Measured from Water Samples}

Seventy-eight water samples were collected at this station from June 2000 through October 2005 (table 6). Samples were analyzed for major ions, nutrients, trace elements, and suspended sediment. Only values for instantaneous streamflow, $\mathrm{SC}$, and SAR are shown; values for the remaining constituents can be accessed at http://waterdata.usgs.gov/mt/nwis.

\section{Estimated Sodium-Adsorption Ratio and Regression Equations for 2004-06}

SAR values for Tongue River below Brandenberg Bridge for the 2004 irrigation season were estimated using continuous SC data and a regression equation (fig. 10A) based on the SC and SAR values from 59 water samples collected from June 2000 through September 2004 (table 6). SAR values for the 2005 irrigation season were estimated using continuous SC and a regression equation (fig. 10B) based on data from all 35 samples from 2004-05 (table 6). Provisional real-time SAR data for 2006 also were estimated using the equation for the 2005 irrigation season (fig. 10B). Comparisons of sample SAR to daily mean estimated SAR for the 2004 and 2005 irrigation seasons are shown on figure 11.

Table 6. Water-sample data collected at Tongue River below Brandenberg Bridge, near Ashland, Montana (06307830), and used in regression equations for estimating sodium-adsorption ratio from specific conductance.

[Abbreviations: $\mathrm{ft}^{3} / \mathrm{s}$, cubic feet per second; SC, specific conductance; $\mu \mathrm{S} / \mathrm{cm}$, microsiemens per centimeter at 25 degrees Celsius; SAR, sodium-adsorption ratio; E, estimated value for streamflow. Symbol: --, data not used]

\begin{tabular}{|c|c|c|c|c|c|c|}
\hline \multirow[b]{2}{*}{ Date } & \multirow{2}{*}{$\begin{array}{l}\text { Streamflow, } \\
\text { in } \mathrm{ft}^{3} / \mathrm{s}\end{array}$} & \multirow{2}{*}{$\begin{array}{c}\text { SC, } \\
\text { in } \mu S / c m\end{array}$} & \multirow[b]{2}{*}{ SAR } & \multicolumn{3}{|c|}{$\begin{array}{l}\text { Sample data used for } \\
\text { regression equation }\end{array}$} \\
\hline & & & & $\begin{array}{l}2004 \\
\text { final }\end{array}$ & $\begin{array}{l}2005 \\
\text { final }\end{array}$ & $\begin{array}{c}2006 \\
\text { provisional } \\
\text { real-time }\end{array}$ \\
\hline $06 / 13 / 2000$ & 1,060 & 400 & 0.64 & $\mathrm{X}$ & -- & -- \\
\hline 06/26/2000 & 744 & 393 & .66 & $X$ & -- & -- \\
\hline 07/19/2000 & 377 & 429 & .72 & $X$ & -- & -- \\
\hline 08/16/2000 & 298 & 469 & .77 & $X$ & -- & -- \\
\hline $10 / 30 / 2000$ & 307 & 684 & .95 & $X$ & -- & -- \\
\hline $11 / 21 / 2000$ & 160 & 786 & 1.17 & $X$ & -- & -- \\
\hline $12 / 28 / 2000$ & 150 & 850 & 1.22 & $X$ & -- & -- \\
\hline 01/11/2001 & 190 & 842 & 1.31 & $X$ & -- & -- \\
\hline $02 / 12 / 2001$ & 140 & 818 & 1.36 & $X$ & -- & -- \\
\hline 03/28/2001 & 132 & 900 & 1.53 & $X$ & -- & -- \\
\hline $04 / 11 / 2001$ & 153 & 910 & 1.58 & $X$ & -- & -- \\
\hline 05/23/2001 & 217 & 776 & 1.18 & $X$ & -- & -- \\
\hline 06/05/2001 & 622 & 555 & .78 & $X$ & -- & -- \\
\hline 07/11/2001 & 120 & 826 & 1.56 & $X$ & -- & -- \\
\hline 08/20/2001 & 103 & 843 & 1.64 & $X$ & -- & -- \\
\hline 09/05/2001 & 215 & 742 & 1.34 & $X$ & -- & -- \\
\hline $10 / 02 / 2001$ & 88 & 849 & 1.76 & $X$ & -- & -- \\
\hline $11 / 20 / 2001$ & 155 & 700 & 1.16 & $X$ & -- & -- \\
\hline $12 / 11 / 2001$ & E95 & 1,025 & 1.77 & $X$ & -- & -- \\
\hline
\end{tabular}


Table 6. Water-sample data collected at Tongue River below Brandenberg Bridge, near Ashland, Montana (06307830), and used in regression equations for estimating sodium-adsorption ratio from specific conductance.

\section{-Continued}

[Abbreviations: $\mathrm{ft}^{3} / \mathrm{s}$, cubic feet per second; SC, specific conductance; $\mu \mathrm{S} / \mathrm{cm}$, microsiemens per centimeter at 25 degrees Celsius; SAR, sodium-adsorption ratio; E, estimated value for streamflow. Symbol: --, data not used]

\begin{tabular}{|c|c|c|c|c|c|c|}
\hline \multirow[b]{2}{*}{ Date } & \multirow{2}{*}{$\begin{array}{l}\text { Streamflow, } \\
\text { in } \mathrm{ft}^{3} / \mathrm{s}\end{array}$} & \multirow{2}{*}{$\begin{array}{c}S C, \\
\text { in } \mu S / c m\end{array}$} & \multirow[b]{2}{*}{ SAR } & \multicolumn{3}{|c|}{$\begin{array}{l}\text { Sample data used for } \\
\text { regression equation }\end{array}$} \\
\hline & & & & $\begin{array}{l}2004 \\
\text { final }\end{array}$ & $\begin{array}{l}2005 \\
\text { final }\end{array}$ & $\begin{array}{c}2006 \\
\text { provisional } \\
\text { real-time }\end{array}$ \\
\hline $01 / 15 / 2002$ & E100 & 944 & 1.57 & $\mathrm{X}$ & -- & -- \\
\hline 02/07/2002 & E90 & 983 & 1.65 & $X$ & -- & -- \\
\hline 03/18/2002 & E70 & 788 & 1.50 & $X$ & -- & -- \\
\hline $04 / 23 / 2002$ & 98 & 942 & 1.83 & $\mathrm{X}$ & -- & -- \\
\hline $05 / 29 / 2002$ & 151 & 805 & 1.40 & $X$ & -- & -- \\
\hline 06/05/2002 & 181 & 780 & 1.24 & $X$ & -- & -- \\
\hline 07/10/2002 & 222 & 630 & 1.09 & $X$ & -- & -- \\
\hline 08/13/2002 & 94 & 722 & 1.49 & $X$ & -- & -- \\
\hline $09 / 10 / 2002$ & 177 & 608 & 1.32 & $X$ & -- & -- \\
\hline $10 / 01 / 2002$ & 122 & 720 & 1.46 & $X$ & -- & -- \\
\hline $11 / 21 / 2002$ & 106 & 937 & 1.69 & $X$ & -- & -- \\
\hline $12 / 05 / 2002$ & 19 & 888 & 1.52 & $X$ & -- & -- \\
\hline 01/07/2003 & E120 & 808 & 1.36 & $\mathrm{X}$ & -- & -- \\
\hline $02 / 05 / 2003$ & 143 & 640 & 1.15 & $X$ & -- & -- \\
\hline 03/06/2003 & E100 & 905 & 1.32 & $X$ & -- & -- \\
\hline $04 / 23 / 2003$ & 133 & 1,067 & 1.88 & $X$ & -- & -- \\
\hline 05/05/2003 & 430 & 772 & 1.20 & $X$ & -- & -- \\
\hline 06/16/2003 & 1,230 & 398 & .59 & $X$ & -- & -- \\
\hline 07/02/2003 & 882 & 381 & .56 & $X$ & -- & -- \\
\hline 08/04/2003 & 355 & 464 & .72 & $X$ & -- & -- \\
\hline 09/02/2003 & 333 & 507 & .73 & $X$ & -- & -- \\
\hline $10 / 07 / 2003$ & 134 & 703 & 1.30 & $X$ & -- & -- \\
\hline $11 / 13 / 2003$ & E150 & 826 & 1.39 & $\mathrm{X}$ & -- & -- \\
\hline $12 / 03 / 2003$ & E130 & 756 & 1.11 & $\mathrm{X}$ & -- & -- \\
\hline $01 / 22 / 2004$ & E120 & 806 & 1.19 & $X$ & $X$ & $X$ \\
\hline 02/04/2004 & E120 & 907 & 1.27 & $\mathrm{X}$ & $\mathrm{X}$ & $\mathrm{X}$ \\
\hline $02 / 18 / 2004$ & E160 & 870 & 1.31 & $X$ & $X$ & $X$ \\
\hline 03/10/2004 & 199 & 720 & 1.28 & $\mathrm{X}$ & $\mathrm{X}$ & $\mathrm{X}$ \\
\hline $03 / 23 / 2004$ & 189 & 808 & 1.32 & $X$ & $\mathrm{X}$ & $\mathrm{X}$ \\
\hline $04 / 14 / 2004$ & 167 & 784 & 1.31 & $X$ & $\mathrm{X}$ & $\mathrm{X}$ \\
\hline $04 / 28 / 2004$ & 137 & 780 & 1.30 & $\mathrm{X}$ & $\mathrm{X}$ & $\mathrm{X}$ \\
\hline $05 / 12 / 2004$ & 179 & 718 & 1.14 & $\mathrm{X}$ & $X$ & $\mathrm{X}$ \\
\hline $05 / 24 / 2004$ & 249 & 702 & 1.12 & $\mathrm{X}$ & $X$ & $\mathrm{X}$ \\
\hline 06/08/2004 & 146 & 764 & 1.42 & $X$ & $X$ & $X$ \\
\hline $06 / 23 / 2004$ & E190 & 712 & 1.26 & $X$ & $X$ & $X$ \\
\hline
\end{tabular}


Table 6. Water-sample data collected at Tongue River below Brandenberg Bridge, near Ashland, Montana (06307830), and used in regression equations for estimating sodium-adsorption ratio from specific conductance.

-Continued

[Abbreviations: $\mathrm{ft}^{3} / \mathrm{s}$, cubic feet per second; SC, specific conductance; $\mu \mathrm{S} / \mathrm{cm}$, microsiemens per centimeter at 25 degrees Celsius; SAR, sodium-adsorption ratio; E, estimated value for streamflow. Symbol: --, data not used]

\begin{tabular}{|c|c|c|c|c|c|c|}
\hline \multirow[b]{2}{*}{ Date } & \multirow[b]{2}{*}{$\begin{array}{l}\text { Streamflow, } \\
\text { in } \mathrm{ft}^{3} / \mathrm{s}\end{array}$} & \multirow[b]{2}{*}{$\begin{array}{c}\text { SC, } \\
\text { in } \mu \mathrm{S} / \mathrm{cm}\end{array}$} & \multirow[b]{2}{*}{ SAR } & \multicolumn{3}{|c|}{$\begin{array}{l}\text { Sample data used for } \\
\text { regression equation }\end{array}$} \\
\hline & & & & $\begin{array}{l}2004 \\
\text { final }\end{array}$ & $\begin{array}{l}2005 \\
\text { final }\end{array}$ & $\begin{array}{c}2006 \\
\text { provisional } \\
\text { real-time }\end{array}$ \\
\hline 07/13/2004 & 189 & 680 & 1.30 & $\mathrm{X}$ & $\mathrm{X}$ & $X$ \\
\hline $07 / 26 / 2004$ & 246 & 670 & 1.06 & $\mathrm{X}$ & $\mathrm{X}$ & $\mathrm{X}$ \\
\hline 08/18/2004 & 174 & 674 & 1.23 & $\mathrm{X}$ & $\mathrm{X}$ & $\mathrm{X}$ \\
\hline 08/23/2004 & 199 & 641 & 1.16 & $\mathrm{X}$ & $\mathrm{X}$ & $\mathrm{X}$ \\
\hline 09/28/2004 & 101 & 780 & 1.41 & $\mathrm{X}$ & $\mathrm{X}$ & $\mathrm{X}$ \\
\hline $10 / 12 / 2004$ & 95 & 786 & 1.52 & -- & $\mathrm{X}$ & $\mathrm{X}$ \\
\hline $11 / 01 / 2004$ & 110 & 768 & 1.49 & -- & $\mathrm{X}$ & $\mathrm{X}$ \\
\hline $12 / 08 / 2004$ & 90 & 910 & 1.52 & -- & $\mathrm{X}$ & $\mathrm{X}$ \\
\hline 02/09/2005 & 60 & 926 & 1.52 & -- & $\mathrm{X}$ & $X$ \\
\hline 03/10/2005 & 92 & 904 & 1.60 & -- & $\mathrm{X}$ & $\mathrm{X}$ \\
\hline 03/22/2005 & 90 & 906 & 1.64 & -- & $\mathrm{X}$ & $X$ \\
\hline 04/06/2005 & 85 & 920 & 1.74 & -- & $\mathrm{X}$ & $X$ \\
\hline $04 / 18 / 2005$ & 80 & 919 & 1.85 & -- & $\mathrm{X}$ & $\mathrm{X}$ \\
\hline 05/04/2005 & 97 & 876 & 1.61 & -- & $\mathrm{X}$ & $\mathrm{X}$ \\
\hline $05 / 16 / 2005$ & 1,130 & 744 & 1.10 & -- & $\mathrm{X}$ & $\mathrm{X}$ \\
\hline 06/07/2005 & 1,000 & 430 & .68 & -- & $\mathrm{X}$ & $X$ \\
\hline $06 / 21 / 2005$ & 1,880 & 337 & .48 & -- & $\mathrm{X}$ & $\mathrm{X}$ \\
\hline $07 / 12 / 2005$ & 510 & 373 & .68 & -- & $\mathrm{X}$ & $X$ \\
\hline $07 / 27 / 2005$ & 420 & 412 & .64 & -- & $\mathrm{X}$ & $\mathrm{X}$ \\
\hline 08/02/2005 & 396 & 408 & .60 & -- & $\mathrm{X}$ & $X$ \\
\hline $08 / 23 / 2005$ & 369 & 480 & .78 & -- & $\mathrm{X}$ & $\mathrm{X}$ \\
\hline 09/06/2005 & 336 & 473 & .71 & -- & $\mathrm{X}$ & $X$ \\
\hline 09/20/2005 & 272 & 530 & .78 & -- & $\mathrm{X}$ & $X$ \\
\hline $10 / 04 / 2005$ & 255 & 561 & .89 & -- & $\mathrm{X}$ & $X$ \\
\hline
\end{tabular}


Tongue River below Brandenberg Bridge

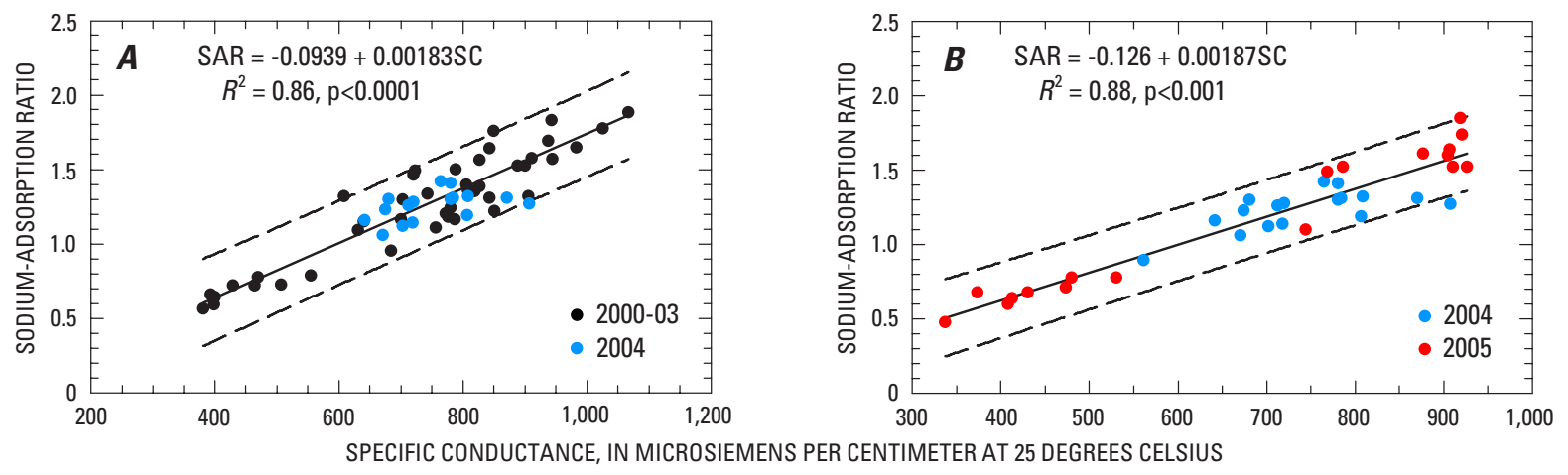

Figure 10. Relation of specific conductance (SC) and sodium-adsorption ratio (SAR) for water-quality samples collected at Tongue River below Brandenberg Bridge, near Ashland, Montana: $A$, Relation used to estimate SAR for the 2004 irrigation season and based on data collected June 2000 through September 2004; $B$, Relation used to estimate SAR for the 2005 irrigation season and based on data collected January 2004 through October 2005. Dashed lines indicate 95-percent prediction interval.

\section{Tongue River below Brandenberg Bridge}
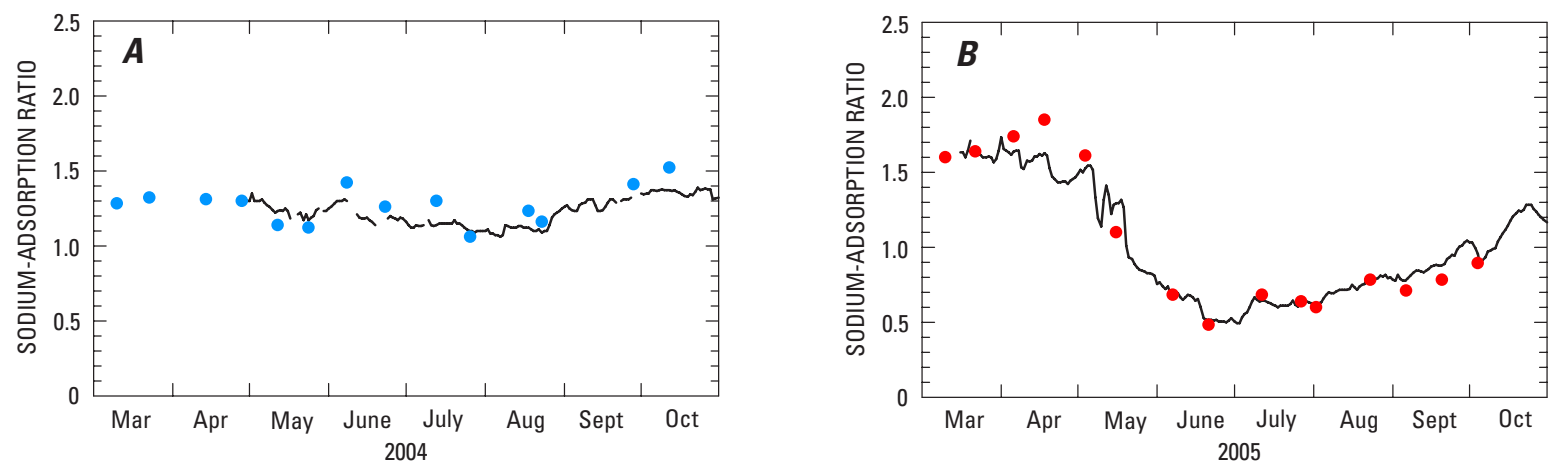

\section{EXPLANATION}

— Sodium-adsorption ratio (SAR) estimated from specific conductance (SC) • Sodium-adsorption ratio (SAR) of samples

Figure 11. Sodium-adsorption ratio (SAR) determined from water-quality samples and estimated from specific conductance (SC) for Tongue River below Brandenberg Bridge, near Ashland, Montana, using the relations shown in figure 10: $A, 2004$ irrigation season; $B, 2005$ irrigation season. 


\section{Tongue River above T\&Y Diversion Dam, near Miles City, Montana}

This station (USGS station 06307990) is located in Custer County, Montana, at a private bridge, 16 miles south of Miles City (fig. 1) and 4.7 miles upstream from an irrigation diversion. The station is at an altitude of 2,480 feet (NGVD 29) and has a drainage area of 4,508 square miles.

\section{Sodium-Adsorption Ratio Measured from Water Samples}

Twenty water samples were collected at this station from November 2004 through October 2005 (table 7). Samples were analyzed for major ions, nutrients, trace elements, and suspended sediment. Only values for instantaneous streamflow,
SC, and SAR are shown; values for the remaining constituents can be accessed at http://waterdata.usgs.gov/mt/nwis.

\section{Estimated Sodium-Adsorption Ratio and Regression Equations for 2005-06}

SAR values for Tongue River above T\&Y Diversion Dam for the 2004 irrigation season were not estimated using continuous SC data and a regression equation based on the SC and SAR because only two water samples were collected in 2004. SAR values for the 2005 irrigation season were estimated using continuous SC and a regression equation (fig. 12) based on data from all 20 samples from 2004-05 (table 7). Provisional real-time SAR data for 2006 also were estimated using this regression equation. A comparison of sample SAR to daily mean estimated SAR for the 2005 irrigation season is shown on figure 13 .

Table 7. Water-sample data collected at Tongue River above T\&Y Diversion Dam, near Miles City, Montana (06307990), and used in regression equations for estimating sodium-adsorption ratio from specific conductance.

[Abbreviations: $\mathrm{ft}^{3} / \mathrm{s}$, cubic feet per second; SC, specific conductance; $\mu \mathrm{S} / \mathrm{cm}$, microsiemens per centimeter at 25 degrees Celsius; SAR, sodium-adsorption ratio; E, estimated value for streamflow. Symbol: --, data not used]

\begin{tabular}{|c|c|c|c|c|c|c|}
\hline \multirow[b]{2}{*}{ Date } & \multirow{2}{*}{$\begin{array}{l}\text { Streamflow, } \\
\text { in } \mathrm{ft}^{3} / \mathrm{s}\end{array}$} & \multirow{2}{*}{$\begin{array}{c}S C, \\
\text { in } \mu S / c m\end{array}$} & \multirow[b]{2}{*}{ SAR } & \multicolumn{3}{|c|}{$\begin{array}{l}\text { Sample data used for } \\
\text { regression equation }\end{array}$} \\
\hline & & & & $\begin{array}{l}2004^{1} \\
\text { final }\end{array}$ & $\begin{array}{l}2005 \\
\text { final }\end{array}$ & $\begin{array}{c}2006 \\
\text { provisional } \\
\text { real-time }\end{array}$ \\
\hline $11 / 01 / 2004$ & 110 & 841 & 1.80 & -- & $\mathrm{X}$ & $X$ \\
\hline $12 / 08 / 2004$ & E80 & 1,000 & 1.82 & -- & $\mathrm{X}$ & $\mathrm{X}$ \\
\hline $01 / 19 / 2005$ & E70 & 990 & 1.81 & -- & $X$ & $X$ \\
\hline 02/09/2005 & E60 & 947 & 1.81 & -- & $X$ & $\mathrm{X}$ \\
\hline 02/22/2005 & 70 & 809 & 1.76 & -- & $X$ & $\mathrm{X}$ \\
\hline 03/10/2005 & E90 & 939 & 1.85 & -- & $X$ & $X$ \\
\hline 03/22/2005 & 89 & 939 & 1.86 & -- & $\mathrm{X}$ & $\mathrm{X}$ \\
\hline 04/06/2005 & 76 & 979 & 2.09 & -- & $X$ & $\mathrm{X}$ \\
\hline 04/18/2005 & 70 & 972 & 2.13 & -- & $\mathrm{X}$ & $\mathrm{X}$ \\
\hline 05/04/2005 & 76 & 926 & 1.83 & -- & $\mathrm{X}$ & $\mathrm{X}$ \\
\hline 05/18/2005 & 1,170 & 738 & 1.19 & -- & $X$ & $\mathrm{X}$ \\
\hline 06/07/2005 & 1,150 & 434 & .67 & -- & $\mathrm{X}$ & $\mathrm{X}$ \\
\hline 06/21/2005 & 1,810 & 351 & .54 & -- & $X$ & $X$ \\
\hline $07 / 13 / 2005$ & 466 & 419 & .81 & -- & $\mathrm{X}$ & $\mathrm{X}$ \\
\hline 07/27/2005 & 337 & 455 & .81 & -- & $\mathrm{X}$ & $\mathrm{X}$ \\
\hline 08/02/2005 & 326 & 453 & .74 & -- & $\mathrm{X}$ & $X$ \\
\hline $08 / 23 / 2005$ & 296 & 512 & .94 & -- & $X$ & $X$ \\
\hline 09/06/2005 & 282 & 525 & .85 & -- & $\mathrm{X}$ & $\mathrm{X}$ \\
\hline 09/20/2005 & 265 & 579 & .95 & -- & $\mathrm{X}$ & $\mathrm{X}$ \\
\hline $10 / 04 / 2005$ & 257 & 602 & 1.18 & -- & $X$ & $X$ \\
\hline
\end{tabular}

${ }^{1}$ Only two samples available in 2004; regression relation is inadequate for estimation of SAR. 


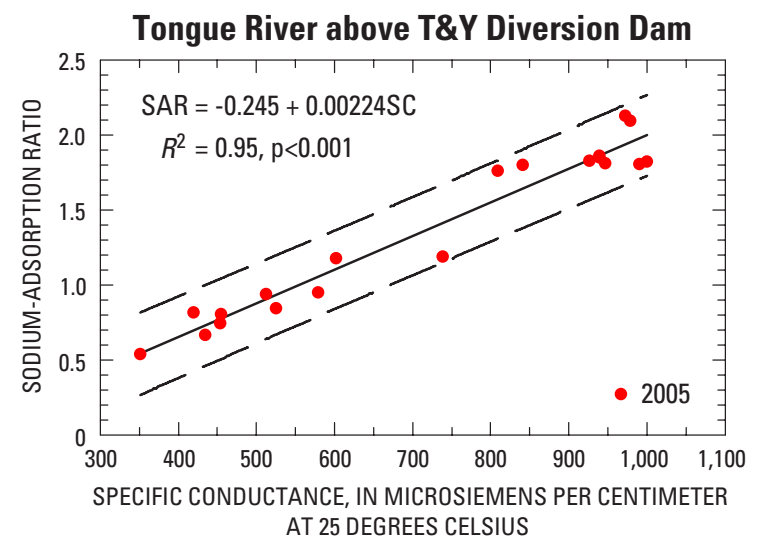

Figure 12. Relation of specific conductance (SC) and sodium-adsorption ratio (SAR) for water-quality samples collected November 2004 through October 2005 at Tongue River above T\&Y Diversion Dam, near Miles City, Montana, and used to estimate SAR for the 2005 irrigation season. Dashed lines indicate 95-percent prediction interval.

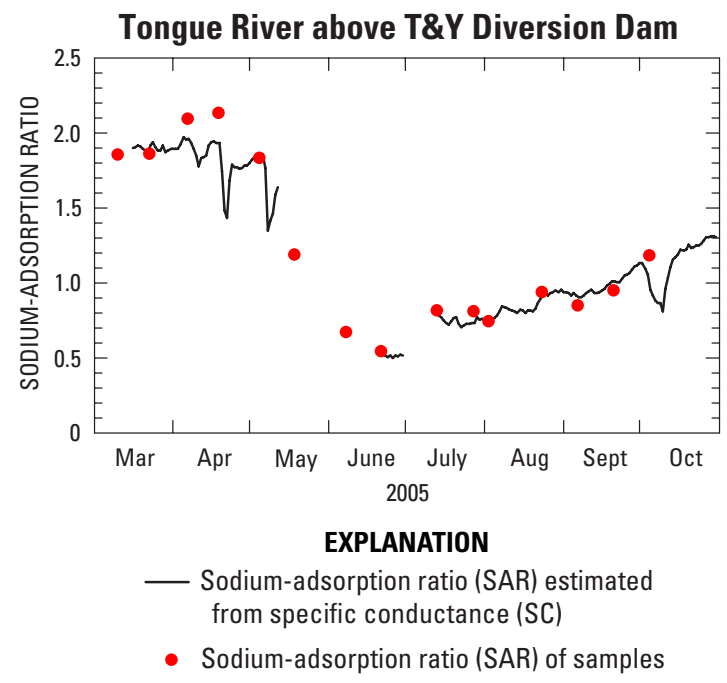

Figure 13. Sodium-adsorption ratio (SAR) determined from water-quality samples and estimated from specific conductance (SC) for Tongue River above T\&Y Diversion Dam, near Miles City, Montana, for the 2005 irrigation season using relation shown in figure 12. 


\section{Tongue River at Miles City, Montana}

This station (USGS station 06308500) is located in Custer County, Montana, 1.5 miles south of Miles City (fig. 1) and 2.3 river miles upstream from the confluence with the Yellowstone River. The station is at an altitude of 2,360 feet (NGVD 29) and has a drainage area of 5,397 square miles.

\section{Sodium-Adsorption Ratio Measured from Water Samples}

Sixty-two water samples were collected at this station from February 1990 through October 2005 (table 8). Samples were analyzed for major ions, nutrients, trace elements, and suspended sediment. Only values for instantaneous streamflow, SC, and SAR are shown; values for the remaining constituents can be accessed at $h t t p: / /$ waterdata.usgs.gov/mt/nwis.

\section{Estimated Sodium-Adsorption Ratio and Regression Equations for 2004-06}

SAR values for Tongue River at Miles City for the 2004 irrigation season were estimated using continuous SC data and a regression equation (fig. 14A) based on the SC and SAR values from 42 water samples collected from February 1990 through August 2004. SAR values for the 2005 irrigation season were estimated using continuous SC and a regression equation (fig. 14B) based on data from 33 samples from 2004-05 (table 8). Provisional real-time SAR data for 2006 also were estimated using the equation for the 2005 irrigation season (fig. 14B). Comparisons of sample SAR to daily mean estimated SAR for the 2004 and 2005 irrigation seasons are shown on figure 15 .

Table 8. Water-sample data collected at Tongue River at Miles City, Montana (06308500), and used in regression equations for estimating sodium-adsorption ratio from specific conductance.

[Abbreviations: $\mathrm{ft}^{3} / \mathrm{s}$, cubic feet per second; SC, specific conductance; $\mu \mathrm{S} / \mathrm{cm}$, microsiemens per centimeter at 25 degrees Celsius; SAR, sodium-adsorption ratio; E, estimated value for streamflow. Symbol: --, data not used]

\begin{tabular}{|c|c|c|c|c|c|c|}
\hline \multirow[b]{2}{*}{ Date } & \multirow{2}{*}{$\begin{array}{l}\text { Streamflow, } \\
\text { in } \mathrm{ft}^{3} / \mathrm{s}\end{array}$} & \multirow{2}{*}{$\begin{array}{c}S C, \\
\text { in } \mu S / c m\end{array}$} & \multirow[b]{2}{*}{ SAR } & \multicolumn{3}{|c|}{$\begin{array}{l}\text { Sample data used for } \\
\text { regression equation }\end{array}$} \\
\hline & & & & $\begin{array}{l}2004 \\
\text { final }\end{array}$ & $\begin{array}{l}2005 \\
\text { final }\end{array}$ & $\begin{array}{c}2006 \\
\text { provisional } \\
\text { real-time }\end{array}$ \\
\hline $02 / 05 / 1990$ & 91 & 989 & 1.47 & $\mathrm{X}$ & -- & -- \\
\hline 06/18/1990 & 1,500 & 350 & .87 & $\mathrm{X}$ & -- & -- \\
\hline 08/13/1990 & 180 & 592 & 1.18 & $\mathrm{X}$ & -- & -- \\
\hline $11 / 13 / 1990$ & 292 & 793 & 1.21 & $\mathrm{X}$ & -- & -- \\
\hline 02/19/1991 & 157 & 773 & 1.44 & $X$ & -- & -- \\
\hline 05/28/1991 & 2,210 & 294 & .68 & $X$ & -- & -- \\
\hline 08/12/1991 & 229 & 529 & 1.02 & $\mathrm{X}$ & -- & -- \\
\hline $11 / 18 / 1991$ & 226 & 751 & 1.48 & $X$ & -- & -- \\
\hline 03/02/1992 & 244 & 807 & 1.31 & $\mathrm{X}$ & -- & -- \\
\hline 06/08/1992 & 76 & 651 & 1.38 & $\mathrm{X}$ & -- & -- \\
\hline 09/01/1992 & 317 & 579 & 1.01 & $X$ & -- & -- \\
\hline $11 / 02 / 1992$ & 294 & 718 & 1.23 & $\mathrm{X}$ & -- & -- \\
\hline $02 / 24 / 1993$ & 146 & 854 & 1.27 & $\mathrm{X}$ & -- & -- \\
\hline 06/09/1993 & 2,550 & 369 & 3.43 & ${ }^{1}--$ & -- & -- \\
\hline $09 / 14 / 1993$ & 167 & 793 & 1.62 & $X$ & -- & -- \\
\hline $11 / 09 / 1993$ & 258 & 818 & 1.49 & $\mathrm{X}$ & -- & -- \\
\hline 03/30/1994 & 487 & 993 & 2.13 & $X$ & -- & -- \\
\hline 06/08/1994 & 242 & 725 & 1.63 & $X$ & -- & -- \\
\hline 09/21/1994 & 28 & 1,060 & 2.69 & $\mathrm{X}$ & -- & -- \\
\hline 06/15/1999 & 2,740 & 313 & .54 & $\mathrm{X}$ & -- & -- \\
\hline
\end{tabular}


Table 8. Water-sample data collected at Tongue River at Miles City, Montana (06308500), and used in regression equations for estimating sodium-adsorption ratio from specific conductance. - Continued

[Abbreviations: $\mathrm{ft}^{3} / \mathrm{s}$, cubic feet per second; SC, specific conductance; $\mu \mathrm{S} / \mathrm{cm}$, microsiemens per centimeter at 25 degrees Celsius; SAR, sodium-adsorption ratio; E, estimated value for streamflow. Symbol: --, data not used]

\begin{tabular}{|c|c|c|c|c|c|c|}
\hline \multirow[b]{2}{*}{ Date } & \multirow[b]{2}{*}{$\begin{array}{l}\text { Streamflow, } \\
\text { in } \mathrm{ft}^{3} / \mathrm{s}\end{array}$} & \multirow[b]{2}{*}{$\begin{array}{c}S C, \\
\text { in } \mu S / c m\end{array}$} & \multirow[b]{2}{*}{ SAR } & \multicolumn{3}{|c|}{$\begin{array}{l}\text { Sample data used for } \\
\text { regression equation }\end{array}$} \\
\hline & & & & $\begin{array}{l}2004 \\
\text { final }\end{array}$ & $\begin{array}{l}2005 \\
\text { final }\end{array}$ & $\begin{array}{c}2006 \\
\text { provisional } \\
\text { real-time }\end{array}$ \\
\hline 09/07/1999 & 196 & 688 & 1.56 & $\mathrm{X}$ & -- & -- \\
\hline $11 / 22 / 1999$ & 234 & 850 & 1.36 & $\mathrm{X}$ & -- & -- \\
\hline 06/07/2000 & 760 & 447 & .80 & $\mathrm{X}$ & -- & -- \\
\hline 06/05/2001 & 171 & 831 & 2.56 & $\mathrm{X}$ & -- & -- \\
\hline 08/29/2001 & 7 & 1,340 & 3.52 & $\mathrm{X}$ & -- & -- \\
\hline $10 / 04 / 2001$ & 27 & 1,340 & 3.66 & $\mathrm{X}$ & -- & -- \\
\hline $06 / 12 / 2002$ & 35 & 1,240 & 3.75 & $\mathrm{X}$ & -- & -- \\
\hline $05 / 21 / 2003$ & 368 & 801 & 1.26 & $\mathrm{X}$ & -- & -- \\
\hline $01 / 23 / 2004$ & E110 & 863 & 1.47 & $\mathrm{X}$ & $\mathrm{X}$ & $\mathrm{X}$ \\
\hline $02 / 04 / 2004$ & E70 & 1,030 & 1.69 & $\mathrm{X}$ & $\mathrm{X}$ & $\mathrm{X}$ \\
\hline $02 / 18 / 2004$ & E90 & 920 & 1.54 & $\mathrm{X}$ & $\mathrm{X}$ & $\mathrm{X}$ \\
\hline $03 / 11 / 2004$ & E300 & 683 & 1.56 & $\mathrm{X}$ & $\mathrm{X}$ & $\mathrm{X}$ \\
\hline 03/23/2004 & 179 & 938 & 1.89 & $\mathrm{X}$ & $\mathrm{X}$ & $X$ \\
\hline $04 / 14 / 2004$ & 133 & 850 & 1.65 & $\mathrm{X}$ & $\mathrm{X}$ & $X$ \\
\hline $04 / 28 / 2004$ & 10 & 1,060 & 2.33 & $\mathrm{X}$ & $\mathrm{X}$ & $\mathrm{X}$ \\
\hline $05 / 13 / 2004$ & 10 & 1,280 & 3.56 & $\mathrm{X}$ & $\mathrm{X}$ & $X$ \\
\hline $05 / 25 / 2004$ & 218 & 585 & 2.09 & $\mathrm{X}$ & $\mathrm{X}$ & $\mathrm{X}$ \\
\hline 06/09/2004 & 16 & 951 & 2.76 & $\mathrm{X}$ & $\mathrm{X}$ & $X$ \\
\hline $06 / 23 / 2004$ & 101 & 804 & 1.69 & $\mathrm{X}$ & $\mathrm{X}$ & $\mathrm{X}$ \\
\hline $07 / 14 / 2004$ & 20 & 1,120 & 3.09 & $\mathrm{X}$ & $\mathrm{X}$ & $\mathrm{X}$ \\
\hline $07 / 26 / 2004$ & 15 & 1,292 & 3.20 & $\mathrm{X}$ & $\mathrm{X}$ & $\mathrm{X}$ \\
\hline 08/18/2004 & 29 & 1,180 & 2.89 & $\mathrm{X}$ & $\mathrm{X}$ & $X$ \\
\hline 08/23/2004 & 21 & 1,153 & 2.95 & $\mathrm{X}$ & $\mathrm{X}$ & $\mathrm{X}$ \\
\hline $10 / 13 / 2004$ & 45 & 1,000 & 2.25 & -- & $\mathrm{X}$ & $\mathrm{X}$ \\
\hline $11 / 02 / 2004$ & 128 & 829 & 2.10 & -- & $\mathrm{X}$ & $\mathrm{X}$ \\
\hline $12 / 09 / 2004$ & E80 & 1,068 & 2.14 & -- & $\mathrm{X}$ & $X$ \\
\hline $02 / 10 / 2005$ & E45 & 1,030 & 2.00 & -- & $\mathrm{X}$ & $X$ \\
\hline 03/09/2005 & 98 & 944 & 2.06 & -- & $\mathrm{X}$ & $\mathrm{X}$ \\
\hline $03 / 23 / 2005$ & 101 & 974 & 2.07 & -- & $\mathrm{X}$ & $X$ \\
\hline 04/07/2005 & 80 & 1,050 & 2.37 & -- & $\mathrm{X}$ & $\mathrm{X}$ \\
\hline $04 / 18 / 2005$ & 80 & 1,020 & 2.42 & -- & $\mathrm{X}$ & $X$ \\
\hline 05/04/2005 & 13 & 1,050 & 2.39 & -- & $\mathrm{X}$ & $\mathrm{X}$ \\
\hline 05/17/2005 & 1,250 & 724 & 1.29 & -- & $X$ & $X$ \\
\hline 06/09/2005 & 3,560 & 407 & 3.71 & -- & ${ }^{1--}$ & 1-- \\
\hline $06 / 22 / 2005$ & 1,860 & 354 & .54 & -- & $\mathrm{X}$ & $X$ \\
\hline $07 / 13 / 2005$ & 304 & 492 & .97 & -- & $\mathrm{X}$ & $\mathrm{X}$ \\
\hline
\end{tabular}


Table 8. Water-sample data collected at Tongue River at Miles City, Montana (06308500), and used in regression equations for estimating sodium-adsorption ratio from specific conductance.-Continued

[Abbreviations: $\mathrm{ft}^{3} / \mathrm{s}$, cubic feet per second; SC, specific conductance; $\mu \mathrm{S} / \mathrm{cm}$, microsiemens per centimeter at 25 degrees Celsius; SAR, sodium-adsorption ratio; E, estimated value for streamflow. Symbol: --, data not used]

\begin{tabular}{|c|c|c|c|c|c|c|}
\hline \multirow[b]{2}{*}{ Date } & \multirow{2}{*}{$\begin{array}{l}\text { Streamflow, } \\
\text { in } \mathrm{ft}^{3} / \mathrm{s}\end{array}$} & \multirow{2}{*}{$\begin{array}{c}S C, \\
\text { in } \mu S / c m\end{array}$} & \multirow[b]{2}{*}{ SAR } & \multicolumn{3}{|c|}{$\begin{array}{l}\text { Sample data used for } \\
\text { regression equation }\end{array}$} \\
\hline & & & & $\begin{array}{l}2004 \\
\text { final }\end{array}$ & $\begin{array}{l}2005 \\
\text { final }\end{array}$ & $\begin{array}{c}2006 \\
\text { provisiona } \\
\text { real-time }\end{array}$ \\
\hline $07 / 27 / 2005$ & 138 & 547 & 1.02 & -- & $X$ & $X$ \\
\hline 08/03/2005 & 175 & 519 & 1.01 & -- & $X$ & $\mathrm{X}$ \\
\hline $08 / 23 / 2005$ & 198 & 495 & 1.38 & -- & $\mathrm{X}$ & $\mathrm{X}$ \\
\hline 09/06/2005 & 164 & 614 & 1.07 & -- & $X$ & $X$ \\
\hline $09 / 20 / 2005$ & 206 & 630 & 1.11 & -- & $\mathrm{X}$ & $\mathrm{X}$ \\
\hline $10 / 05 / 2005$ & 353 & 613 & 2.04 & -- & $X$ & $X$ \\
\hline
\end{tabular}

${ }^{1}$ Data outlier was not used in regression analysis. 
Tongue River at Miles City

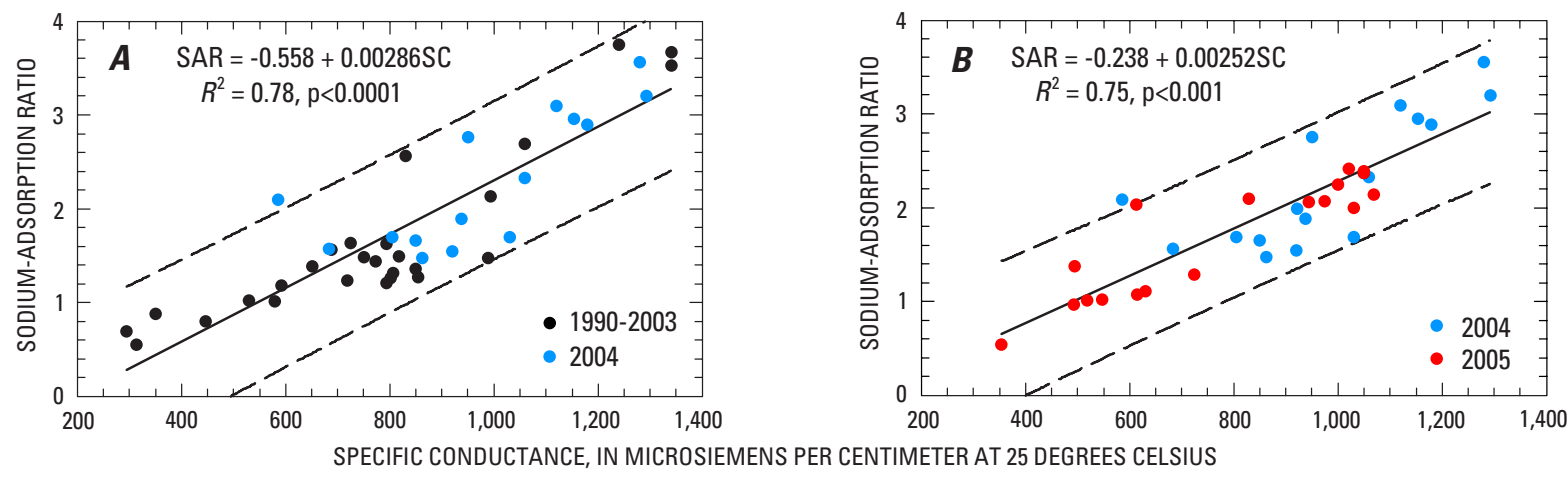

Figure 14. Relation of specific conductance (SC) and sodium-adsorption ratio (SAR) for water-quality samples collected at Tongue River at Miles City, Montana: $A$, Relation used to estimate SAR for the 2004 irrigation season and based on data collected February 1990 through August 2004; B, Relation used to estimate SAR for the 2005 irrigation season and based on data collected January 2004 through October 2005. Dashed lines indicate 95-percent prediction interval.

Tongue River at Miles City
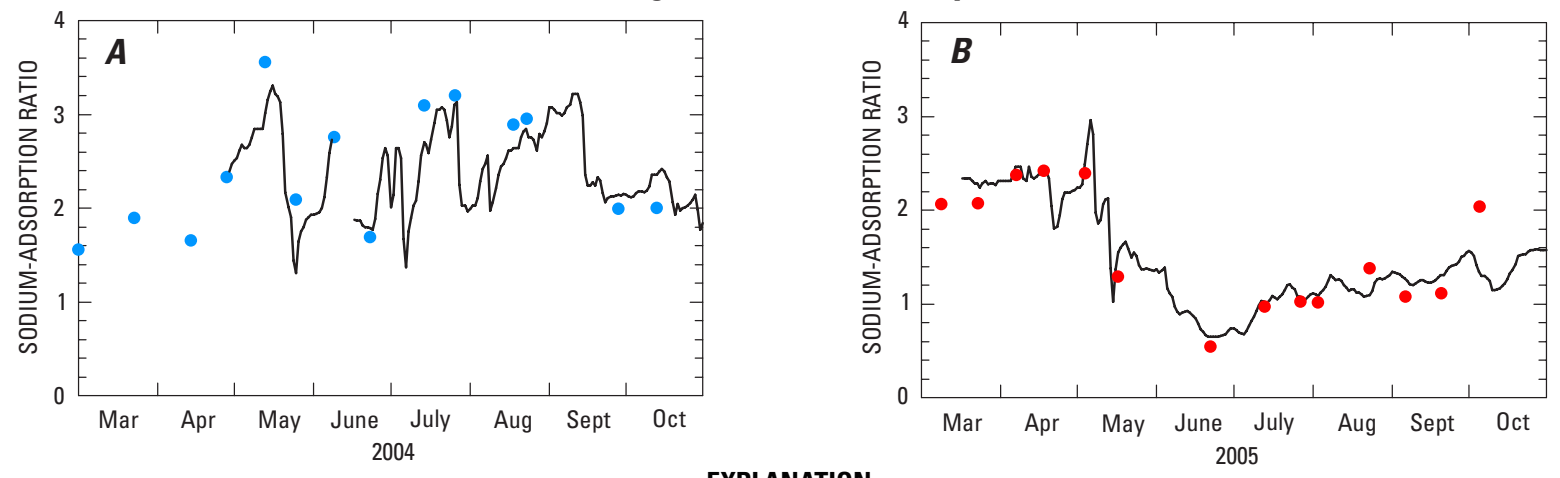

EXPLANATION

— Sodium-adsorption ratio (SAR) estimated from specific conductance (SC) • Sodium-adsorption ratio (SAR) of samples

Figure 15. Sodium-adsorption ratio (SAR) determined from water-quality samples and estimated from specific conductance (SC) for Tongue River at Miles City, Montana, using relations shown in figure 14: $A, 2004$ irrigation season; $B, 2005$ irrigation season. 


\section{Goose Creek near Acme, Wyoming}

This station (USGS station 06305700) is located in Sheridan County, Wyoming, 1.6 miles south of Acme (fig. 1). The station is at an altitude of 3,620 feet (NGVD 29) and has a drainage area of 413 square miles.

\section{Sodium-Adsorption Ratio Measured from Water Samples}

Twenty-three water samples were collected at this station from February 2004 through October 2005 (table 9). Samples were analyzed for major ions, nutrients, trace elements, and suspended sediment. Only values for instantaneous streamflow, SC, and SAR are shown; values for the remaining constituents can be accessed at $h$ ttp://waterdata.usgs.gov/wy/nwis.

\section{Estimated Sodium-Adsorption Ratio and Regression Equations for 2004-06}

SAR values for Goose Creek near Acme for the 2004 irrigation season were estimated using continuous SC data and a regression equation (fig. 16A) based on the SC and SAR values from 10 water samples collected from February 2004 through August 2004 (table 9). SAR values for the 2005 irrigation season were estimated using continuous $\mathrm{SC}$ and a regression equation (fig. 16B) based on data from 23 samples from 2004-05 (table 9). Provisional real-time SAR data for 2006 also were estimated using the equation for the 2005 irrigation season (fig 16B). Comparisons of sample SAR to daily mean estimated SAR for the 2004 and 2005 irrigation seasons are shown on figure 17.

Table 9. Water-sample data collected at Goose Creek near Acme, Wyoming (06305700), and used in regression equations for estimating sodium-adsorption ratio from specific conductance.

[Abbreviations: $\mathrm{ft}^{3} / \mathrm{s}$, cubic feet per second; SC, specific conductance; $\mu \mathrm{S} / \mathrm{cm}$, microsiemens per centimeter at 25 degrees Celsius; SAR, sodium-adsorption ratio; E, estimated value for streamflow. Symbol: --, data not used]

\begin{tabular}{|c|c|c|c|c|c|c|}
\hline \multirow[b]{2}{*}{ Date } & \multirow[b]{2}{*}{$\begin{array}{l}\text { Streamflow, } \\
\text { in } \mathrm{ft}^{3} / \mathrm{s}\end{array}$} & \multirow[b]{2}{*}{$\begin{array}{c}\text { SC, } \\
\text { in } \mu \mathrm{S} / \mathrm{cm}\end{array}$} & \multirow[b]{2}{*}{ SAR } & \multicolumn{3}{|c|}{$\begin{array}{l}\text { Sample data used for } \\
\text { regression equation }\end{array}$} \\
\hline & & & & $\begin{array}{l}2004 \\
\text { final }\end{array}$ & $\begin{array}{l}2005 \\
\text { final }\end{array}$ & $\begin{array}{c}2006 \\
\text { provisional } \\
\text { real-time }\end{array}$ \\
\hline 02/03/2004 & E57 & 643 & 0.56 & $\mathrm{X}$ & $\mathrm{X}$ & $\mathrm{X}$ \\
\hline 03/08/2004 & E62 & 610 & .61 & $\mathrm{X}$ & $\mathrm{X}$ & $X$ \\
\hline $04 / 14 / 2004$ & 78 & 553 & .55 & $\mathrm{X}$ & $\mathrm{X}$ & $X$ \\
\hline $04 / 27 / 2004$ & 29 & 726 & .72 & $\mathrm{X}$ & $\mathrm{X}$ & $\mathrm{X}$ \\
\hline $05 / 14 / 2004$ & 49 & 546 & .59 & $\mathrm{X}$ & $\mathrm{X}$ & $\mathrm{X}$ \\
\hline $05 / 26 / 2004$ & 52 & 610 & .62 & $\mathrm{X}$ & $\mathrm{X}$ & $\mathrm{X}$ \\
\hline 06/09/2004 & 98 & 336 & .44 & $\mathrm{X}$ & $\mathrm{X}$ & $\mathrm{X}$ \\
\hline $06 / 22 / 2004$ & 44 & 725 & .68 & $\mathrm{X}$ & $\mathrm{X}$ & $\mathrm{X}$ \\
\hline $07 / 15 / 2004$ & 34 & 670 & .66 & $\mathrm{X}$ & $\mathrm{X}$ & $\mathrm{X}$ \\
\hline $08 / 17 / 2004$ & 22 & 744 & .77 & $\mathrm{X}$ & $\mathrm{X}$ & $\mathrm{X}$ \\
\hline $10 / 14 / 2004$ & 58 & 772 & .65 & -- & $\mathrm{X}$ & $\mathrm{X}$ \\
\hline $12 / 02 / 2004$ & E52 & 700 & .58 & -- & $\mathrm{X}$ & $\mathrm{X}$ \\
\hline 02/09/2005 & E50 & 771 & .67 & -- & $\mathrm{X}$ & $X$ \\
\hline 03/09/2005 & 57 & 704 & .63 & -- & $\mathrm{X}$ & $\mathrm{X}$ \\
\hline 04/06/2005 & 50 & 569 & .57 & -- & $\mathrm{X}$ & $X$ \\
\hline $04 / 20 / 2005$ & 37 & 749 & .72 & -- & $\mathrm{X}$ & $\mathrm{X}$ \\
\hline 05/03/2005 & 43 & 716 & 1.18 & -- & $\mathrm{X}$ & $\mathrm{X}$ \\
\hline $05 / 12 / 2005$ & 1,350 & 423 & .46 & -- & $\mathrm{X}$ & $X$ \\
\hline 06/09/2005 & 580 & 217 & .29 & -- & $\mathrm{X}$ & $\mathrm{X}$ \\
\hline $06 / 22 / 2005$ & 891 & 130 & .23 & -- & $\mathrm{X}$ & $X$ \\
\hline $07 / 14 / 2005$ & 100 & 588 & .53 & -- & $\mathrm{X}$ & $\mathrm{X}$ \\
\hline 08/04/2005 & 69 & 566 & .53 & -- & $\mathrm{X}$ & $X$ \\
\hline $10 / 19 / 2005$ & 116 & 664 & .54 & -- & $X$ & $\mathrm{X}$ \\
\hline
\end{tabular}


Goose Creek near Acme

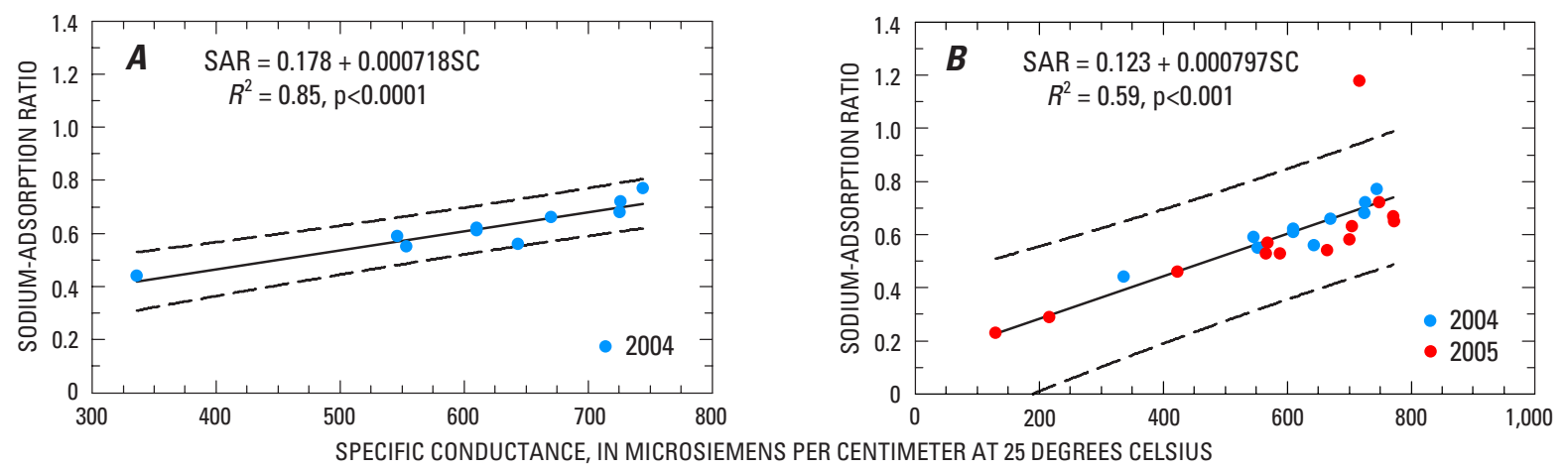

Figure 16. Relation of specific conductance (SC) and sodium-adsorption ratio (SAR) for water-quality samples collected at Goose Creek near Acme, Wyoming: $A$, Relation used to estimate SAR for the 2004 irrigation season and based on data collected February through August 2004; B, Relation used to estimate SAR for the 2005 irrigation season and based on data collected February 2004 through October 2005. Dashed lines indicate 95-percent prediction interval.
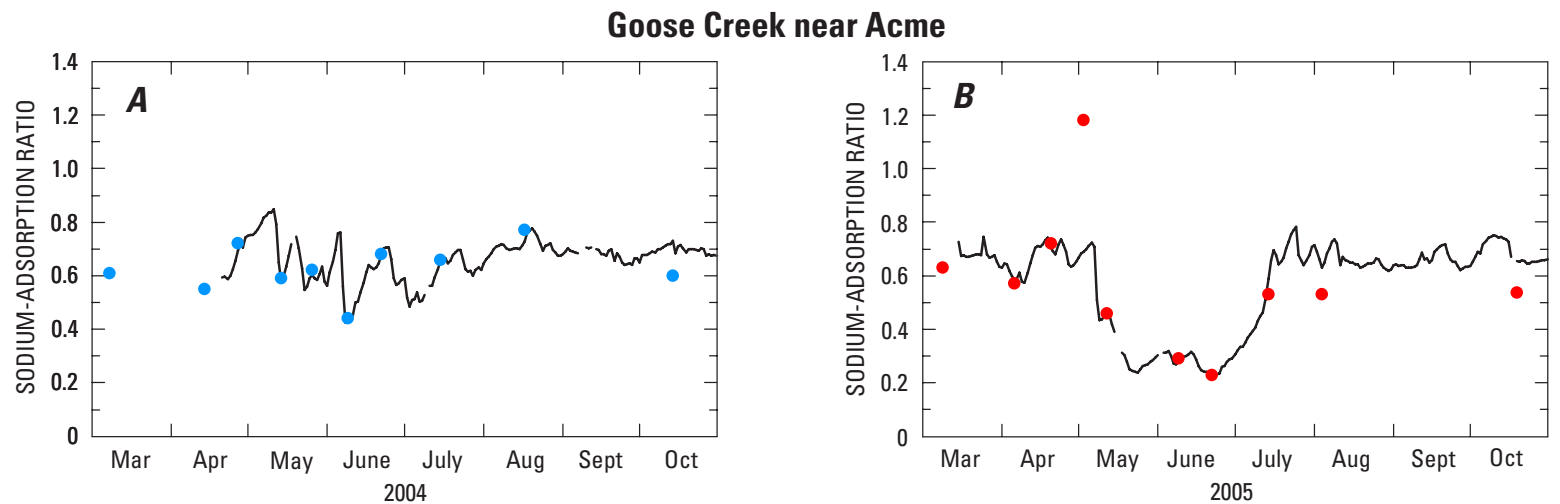

\section{EXPLANATION}

— Sodium-adsorption ratio (SAR) estimated from specific conductance (SC) • Sodium-adsorption ratio (SAR) of samples

Figure 17. Sodium-adsorption ratio (SAR) determined from water-quality samples and estimated from specific conductance (SC) for Goose Creek near Acme, Wyoming, using relations shown in figure 16: $A$, 2004 irrigation season; $B, 2005$ irrigation season. 


\section{Prairie Dog Creek near Acme, Wyoming}

This station (USGS station 06306250) is located in Sheridan County, Wyoming, 7.6 miles northeast of Acme (fig. 1). The station is at an altitude of 3,450 feet (NGVD 29) and has a drainage area of 358 square miles.

\section{Sodium-Adsorption Ratio Measured from Water Samples}

Sixty-eight water samples were collected at this station from May 2000 through October 2005 (table 10). Samples were analyzed for major ions, nutrients, trace elements, and suspended sediment. Only values for instantaneous streamflow, SC, and SAR are shown; values for the remaining constituents can be accessed at $h t t p: / / w a t e r d a t a . u s g s . g o v / w y / n w i s$.

\section{Estimated Sodium-Adsorption Ratio and Regression Equations for 2004-06}

SAR values for Prairie Dog Creek near Acme for the 2004 irrigation season were estimated using continuous SC data and a regression equation (fig. 18A) based on the SC and SAR values from 33 water samples collected from May 2000 through September 2004. These 2000-2004 data were segregated by season, and only the data for April-October were used to develop the SC-SAR equation. Data for November-March were not used to develop the equation, but are presented (table 10, fig. 18) to illustrate seasonal differences. SAR values for the 2005 irrigation season were estimated using continuous $\mathrm{SC}$ and a regression equation (fig. 18B) based on data from 28 samples from December 2003 through October 2005 (table 10). Seasonal differences were not discernible in these data; therefore, the data were not segregated as had been done for the SC-SAR equation for the 2004 irrigation season. Provisional real-time SAR data for 2006 also were estimated using the equation for the 2005 irrigation season (fig. 18B). Comparisons of sample SAR to daily mean estimated SAR for the 2004 and 2005 irrigation seasons are shown on figure 19 .

Table 10. Water-sample data collected at Prairie Dog Creek near Acme, Wyoming (06306250), and used in regression equations for estimating sodium-adsorption ratio from specific conductance.

[Abbreviations: $\mathrm{ft}^{3} / \mathrm{s}$, cubic feet per second; SC, specific conductance; $\mu \mathrm{S} / \mathrm{cm}$, microsiemens per centimeter at 25 degrees Celsius; SAR, sodium-adsorption ratio; E, estimated value for streamflow. Symbol: --, data not used]

\begin{tabular}{|c|c|c|c|c|c|c|}
\hline \multirow[b]{2}{*}{ Date } & \multirow{2}{*}{$\begin{array}{l}\text { Streamflow, } \\
\text { in } \mathrm{ft}^{3} / \mathrm{s}\end{array}$} & \multirow{2}{*}{$\begin{array}{c}\mathrm{SC}, \\
\text { in } \mu \mathrm{S} / \mathrm{cm}\end{array}$} & \multirow[b]{2}{*}{ SAR } & \multicolumn{3}{|c|}{$\begin{array}{l}\text { Sample data used for } \\
\text { regression equation }\end{array}$} \\
\hline & & & & $\begin{array}{c}2004 \\
\text { final }^{1}\end{array}$ & $\begin{array}{l}2005 \\
\text { final }\end{array}$ & $\begin{array}{c}2006 \\
\text { provisional } \\
\text { real-time }\end{array}$ \\
\hline $05 / 15 / 2000$ & 12 & 1,400 & 1.56 & $X$ & -- & -- \\
\hline $08 / 02 / 2000$ & 9 & 1,780 & 1.80 & $X$ & -- & -- \\
\hline $10 / 24 / 2000$ & 27 & 1,390 & 1.07 & $\mathrm{X}$ & -- & -- \\
\hline $11 / 28 / 2000$ & 15 & 1,430 & 1.04 & -- & -- & -- \\
\hline $12 / 14 / 2000$ & 11 & 1,640 & 1.18 & -- & -- & -- \\
\hline $01 / 11 / 2001$ & E17 & 1,491 & 1.17 & -- & -- & -- \\
\hline $02 / 13 / 2001$ & 16 & 1,600 & 1.24 & -- & -- & -- \\
\hline 03/13/2001 & 63 & 993 & 1.00 & -- & -- & -- \\
\hline $04 / 11 / 2001$ & 20 & 1,470 & 1.28 & $\mathrm{X}$ & -- & -- \\
\hline 05/09/2001 & 28 & 1,407 & 1.56 & $\mathrm{X}$ & -- & -- \\
\hline 06/08/2001 & 1.8 & 1,559 & 1.72 & $\mathrm{X}$ & -- & -- \\
\hline $07 / 19 / 2001$ & 5.1 & 1,870 & 1.94 & $X$ & -- & -- \\
\hline $08 / 15 / 2001$ & 3.3 & 2,179 & 2.44 & $\mathrm{X}$ & -- & -- \\
\hline $09 / 12 / 2001$ & 15 & 1,330 & 1.21 & $X$ & -- & -- \\
\hline $10 / 12 / 2001$ & 19 & 1,210 & .94 & $\mathrm{X}$ & -- & -- \\
\hline $11 / 15 / 2001$ & 13 & 1,530 & 1.19 & -- & -- & -- \\
\hline $12 / 11 / 2001$ & 12 & 1,505 & 1.18 & -- & -- & -- \\
\hline
\end{tabular}


Table 10. Water-sample data collected at Prairie Dog Creek near Acme, Wyoming (06306250), and used in regression equations for estimating sodium-adsorption ratio from specific conductance. - Continued

[Abbreviations: $\mathrm{ft}^{3} / \mathrm{s}$, cubic feet per second; SC, specific conductance; $\mu \mathrm{S} / \mathrm{cm}$, microsiemens per centimeter at 25 degrees Celsius; SAR, sodium-adsorption ratio; E, estimated value for streamflow. Symbol: --, data not used]

\begin{tabular}{|c|c|c|c|c|c|c|}
\hline \multirow[b]{2}{*}{ Date } & \multirow[b]{2}{*}{$\begin{array}{l}\text { Streamflow, } \\
\text { in } \mathrm{ft}^{3} / \mathrm{s}\end{array}$} & \multirow[b]{2}{*}{$\begin{array}{c}S C, \\
\text { in } \mu S / c m\end{array}$} & \multirow[b]{2}{*}{ SAR } & \multicolumn{3}{|c|}{$\begin{array}{l}\text { Sample data used for } \\
\text { regression equation }\end{array}$} \\
\hline & & & & $\begin{array}{c}2004 \\
\text { final' }\end{array}$ & $\begin{array}{l}2005 \\
\text { final }\end{array}$ & $\begin{array}{c}2006 \\
\text { provisional } \\
\text { real-time }\end{array}$ \\
\hline 01/09/2002 & 8.1 & 1,480 & 1.13 & -- & -- & -- \\
\hline $02 / 14 / 2002$ & 10 & 1,460 & 1.20 & -- & -- & -- \\
\hline 03/19/2002 & 17 & 1,360 & 1.18 & -- & -- & -- \\
\hline $04 / 10 / 2002$ & 68 & 1,030 & 1.07 & $\mathrm{X}$ & -- & -- \\
\hline 05/08/2002 & 19 & 1,687 & 1.54 & $X$ & -- & -- \\
\hline $06 / 12 / 2002$ & 2.3 & 2,060 & 2.20 & $\mathrm{X}$ & -- & -- \\
\hline 07/08/2002 & 2.5 & 2,280 & 2.49 & $\mathrm{X}$ & -- & -- \\
\hline 08/14/2002 & 19 & 1,170 & 1.14 & $\mathrm{X}$ & -- & -- \\
\hline 09/11/2002 & 51 & 739 & .65 & $\mathrm{X}$ & -- & -- \\
\hline $10 / 07 / 2002$ & 36 & 962 & .85 & $\mathrm{X}$ & -- & -- \\
\hline $11 / 18 / 2002$ & 17 & 1,450 & 1.20 & -- & -- & -- \\
\hline $12 / 12 / 2002$ & 12 & 1,610 & 1.20 & -- & -- & -- \\
\hline 01/29/2003 & 9.8 & 1,463 & 1.10 & -- & -- & -- \\
\hline $02 / 27 / 2003$ & 8.2 & 1,940 & 1.49 & -- & -- & -- \\
\hline $03 / 25 / 2003$ & 80 & 1,290 & 1.20 & -- & -- & -- \\
\hline $04 / 25 / 2003$ & 17 & 1,520 & 1.41 & $\mathrm{X}$ & -- & -- \\
\hline $05 / 21 / 2003$ & 42 & 1,010 & 1.08 & $X$ & -- & -- \\
\hline $06 / 17 / 2003$ & 37 & 942 & .91 & $\mathrm{X}$ & -- & -- \\
\hline $07 / 30 / 2003$ & 2.6 & 1,960 & 2.14 & $\mathrm{X}$ & -- & -- \\
\hline 08/26/2003 & 12 & 1,240 & 1.16 & $\mathrm{X}$ & -- & -- \\
\hline 09/22/2003 & 57 & 642 & .57 & $\mathrm{X}$ & -- & -- \\
\hline $10 / 21 / 2003$ & 20 & 1,330 & 1.09 & $X$ & -- & -- \\
\hline $11 / 20 / 2003$ & 17 & 1,470 & 1.11 & -- & -- & -- \\
\hline $12 / 18 / 2003$ & 9.6 & 1,490 & 1.11 & -- & $\mathrm{X}$ & $\mathrm{X}$ \\
\hline $01 / 22 / 2004$ & 11 & 1,450 & 1.03 & -- & $\mathrm{X}$ & $\mathrm{X}$ \\
\hline $02 / 04 / 2004$ & 10 & 1,617 & 1.31 & -- & $\mathrm{X}$ & $X$ \\
\hline 02/19/2004 & 12 & 1,410 & 1.15 & -- & $\mathrm{X}$ & $X$ \\
\hline 03/09/2004 & E23 & 1,560 & 1.45 & -- & $\mathrm{X}$ & $\mathrm{X}$ \\
\hline $04 / 14 / 2004$ & 11 & 1,330 & 1.46 & $\mathrm{X}$ & $\mathrm{X}$ & $X$ \\
\hline $04 / 27 / 2004$ & 6 & 1,460 & 1.75 & $X$ & $\mathrm{X}$ & $\mathrm{X}$ \\
\hline 05/13/2004 & 6.5 & 2,076 & 2.54 & $X$ & $\mathrm{X}$ & $X$ \\
\hline $05 / 26 / 2004$ & 3.5 & 1,907 & 2.00 & $\mathrm{X}$ & $\mathrm{X}$ & $\mathrm{X}$ \\
\hline 06/09/2004 & 1.2 & 2,512 & 2.71 & $X$ & $\mathrm{X}$ & $X$ \\
\hline $06 / 21 / 2004$ & 11 & 1,670 & 1.70 & $\mathrm{X}$ & $\mathrm{X}$ & $X$ \\
\hline $07 / 14 / 2004$ & 17 & 944 & .97 & $X$ & $\mathrm{X}$ & $X$ \\
\hline 08/18/2004 & 9.5 & 1,395 & 1.27 & $X$ & $\mathrm{X}$ & $\mathrm{X}$ \\
\hline
\end{tabular}


Table 10. Water-sample data collected at Prairie Dog Creek near Acme, Wyoming (06306250), and used in regression equations for estimating sodium-adsorption ratio from specific conductance. - Continued

[Abbreviations: $\mathrm{ft}^{3} / \mathrm{s}$, cubic feet per second; SC, specific conductance; $\mu \mathrm{S} / \mathrm{cm}$, microsiemens per centimeter at 25 degrees Celsius; SAR, sodium-adsorption ratio; E, estimated value for streamflow. Symbol: --, data not used]

\begin{tabular}{|c|c|c|c|c|c|c|}
\hline \multirow[b]{2}{*}{ Date } & \multirow[b]{2}{*}{$\begin{array}{l}\text { Streamflow, } \\
\text { in } \mathrm{ft}^{3} / \mathrm{s}\end{array}$} & \multirow[b]{2}{*}{$\begin{array}{c}\text { SC, } \\
\text { in } \mu S / c m\end{array}$} & \multirow[b]{2}{*}{ SAR } & \multicolumn{3}{|c|}{$\begin{array}{l}\text { Sample data used for } \\
\text { regression equation }\end{array}$} \\
\hline & & & & $\begin{array}{l}2004 \\
\text { final' }\end{array}$ & $\begin{array}{l}2005 \\
\text { final }\end{array}$ & $\begin{array}{c}2006 \\
\text { provisional } \\
\text { real-time }\end{array}$ \\
\hline $09 / 15 / 2004$ & 19 & 1,141 & .99 & $\mathrm{X}$ & $X$ & $\mathrm{X}$ \\
\hline $10 / 13 / 2004$ & 23 & 1,158 & .98 & -- & $\mathrm{X}$ & $X$ \\
\hline $11 / 03 / 2004$ & 19 & 1,336 & 1.11 & -- & $X$ & $\mathrm{X}$ \\
\hline $12 / 02 / 2004$ & E18 & 1,430 & .97 & -- & $\mathrm{X}$ & $\mathrm{X}$ \\
\hline $02 / 09 / 2005$ & E12 & 1,718 & 1.57 & -- & $\mathrm{X}$ & $\mathrm{X}$ \\
\hline 03/08/2005 & 14 & 1,585 & 1.46 & -- & $\mathrm{X}$ & $\mathrm{X}$ \\
\hline $04 / 06 / 2005$ & 9.8 & 1,460 & 1.38 & -- & $\mathrm{X}$ & $\mathrm{X}$ \\
\hline $04 / 19 / 2005$ & 9 & 1,605 & 1.60 & -- & $\mathrm{X}$ & $\mathrm{X}$ \\
\hline 05/03/2005 & 12 & 1,791 & 1.70 & -- & $\mathrm{X}$ & $\mathrm{X}$ \\
\hline 05/17/2005 & 102 & 980 & 1.00 & -- & $\mathrm{X}$ & $\mathrm{X}$ \\
\hline 06/07/2005 & 23 & 1,397 & 1.37 & -- & $\mathrm{X}$ & $\mathrm{X}$ \\
\hline $06 / 22 / 2005$ & 44 & 902 & .93 & -- & $\mathrm{X}$ & $\mathrm{X}$ \\
\hline $07 / 14 / 2005$ & 13 & 1,395 & 1.38 & -- & $\mathrm{X}$ & $\mathrm{X}$ \\
\hline 08/10/2005 & 35 & 807 & .79 & -- & $\mathrm{X}$ & $\mathrm{X}$ \\
\hline $10 / 18 / 2005$ & 28 & 1,103 & 1.03 & -- & $\mathrm{X}$ & $X$ \\
\hline
\end{tabular}

${ }^{1}$ Winter data (November-March) not used to develop 2004 regression equation. 
Prairie Dog Creek near Acme

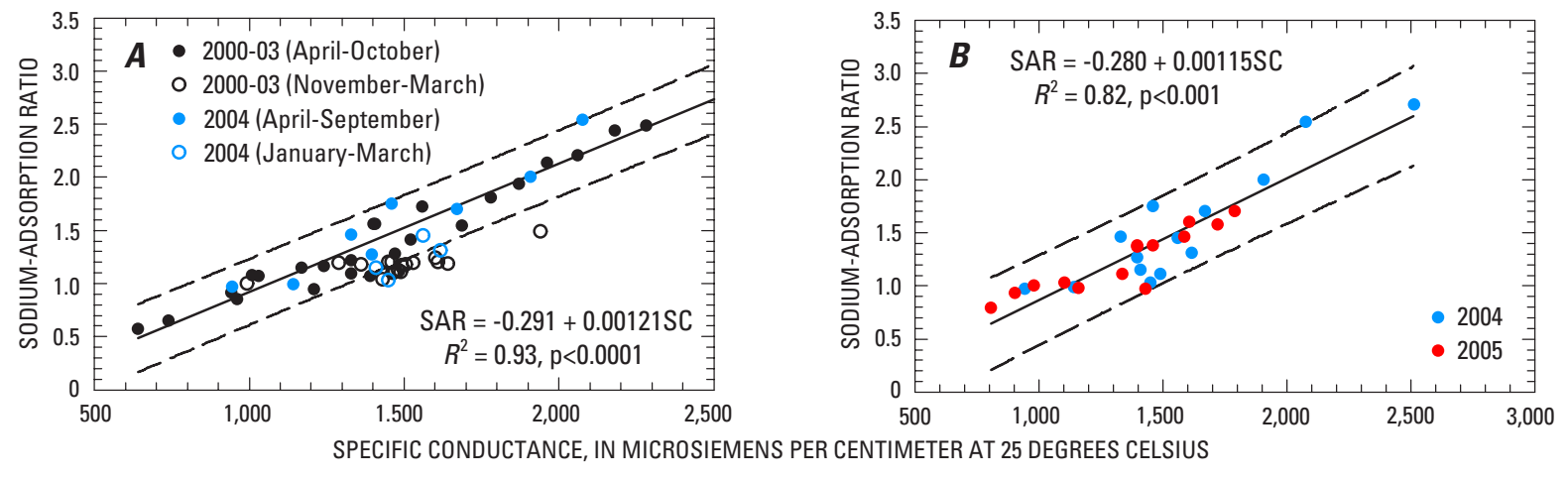

Figure 18. Relation of specific conductance (SC) and sodium-adsorption ratio (SAR) for water-quality samples collected at Prairie Dog Creek near Acme, Wyoming: A, Relation used to estimate SAR for the 2004 irrigation season and based on data collected May 2000 through September 2004; $B$, Relation used to estimate SAR for the 2005 irrigation season and based on data collected December 2003 through October 2005. Dashed lines indicate 95-percent prediction interval.
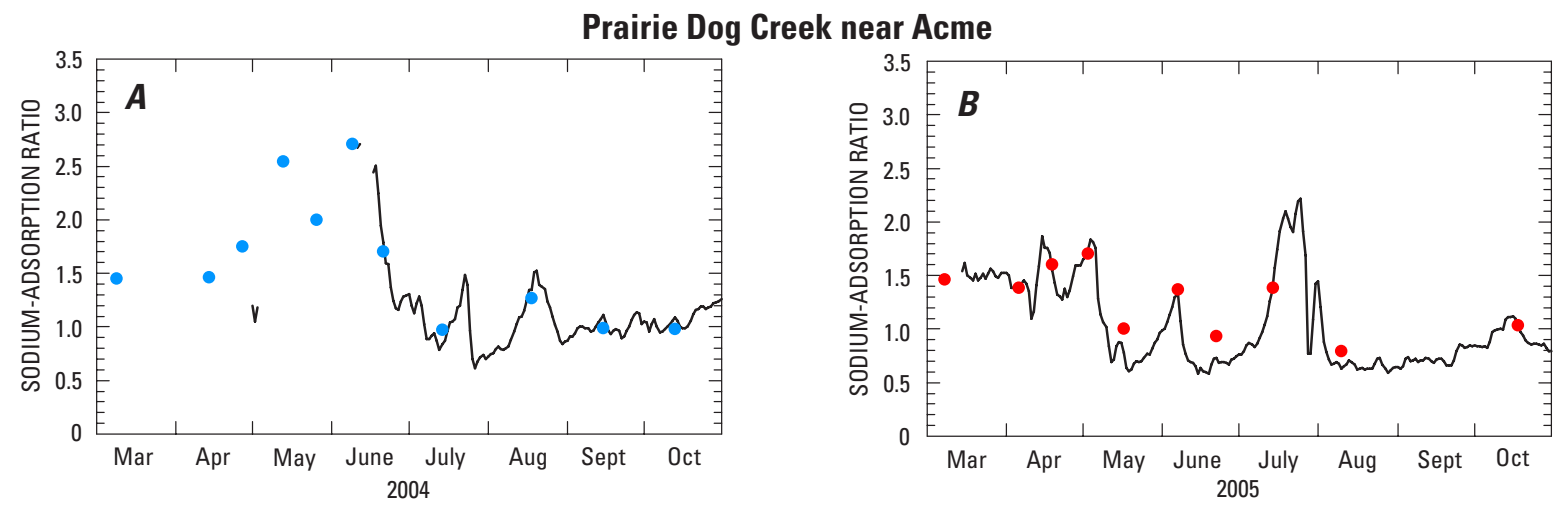

\section{EXPLANATION}

— Sodium-adsorption ratio (SAR) estimated from specific conductance (SC) • • Sodium-adsorption ratio (SAR) of samples

Figure 19. Sodium-adsorption ratio (SAR) determined from water-quality samples and estimated from specific conductance (SC) for Prairie Dog Creek near Acme, Wyoming, using relations shown in figure 18: $A, 2004$ irrigation season; $B, 2005$ irrigation season. 


\section{Hanging Woman Creek near Birney, Montana}

This station (USGS station 06307600) is located in Rosebud County, Montana, 1.9 miles south of Birney (fig. 1). The station is at an altitude of 3,180 feet (NGVD 29) and has a drainage area of 470 square miles.

\section{Sodium-Adsorption Ratio Measured from Water Samples}

Forty-seven water samples were collected at this station from March 1990 through July 2005 (table 11). Samples were analyzed for major ions, nutrients, trace elements, and suspended sediment. Only values for instantaneous streamflow, SC, and SAR are shown; values for the remaining constituents can be accessed at http://waterdata.usgs.gov/mt/nwis.

\section{Estimated Sodium-Adsorption Ratio and Regression Equations for 2004-06}

SAR values for Hanging Woman Creek near Birney for the 2004 irrigation season were estimated using continuous SC data and a regression equation (fig. 20A) based on the SC and SAR values from 36 water samples collected from March 1990 through June 2004 (table 11). SAR values for the 2005 irrigation season were estimated using continuous SC and a regression equation (fig. 20B) based on data from 22 samples from October 2003 through July 2005 (table 11). Provisional real-time SAR data for 2006 also were estimated using the equation for the 2005 irrigation season (fig. 20B). Comparisons of sample SAR to daily mean estimated SAR for the 2004 and 2005 irrigation seasons are shown on figure 21.

Table 11. Water-sample data collected at Hanging Woman Creek near Birney, Montana (06307600), and used in regression equations for estimating sodium-adsorption ratio from specific conductance.

[Abbreviations: $\mathrm{ft}^{3} / \mathrm{s}$, cubic feet per second; SC, specific conductance; $\mu \mathrm{S} / \mathrm{cm}$, microsiemens per centimeter at 25 degrees Celsius; SAR, sodium-adsorption ratio; E, estimated value for streamflow. Symbol: --, data not used]

\begin{tabular}{|c|c|c|c|c|c|c|}
\hline \multirow[b]{2}{*}{ Date } & \multirow{2}{*}{$\begin{array}{l}\text { Streamflow, } \\
\text { in } \mathrm{ft}^{3} / \mathrm{s}\end{array}$} & \multirow{2}{*}{$\begin{array}{c}S C, \\
\text { in } \mu S / c m\end{array}$} & \multirow[b]{2}{*}{ SAR } & \multicolumn{3}{|c|}{$\begin{array}{l}\text { Sample data used for } \\
\text { regression equation }\end{array}$} \\
\hline & & & & $\begin{array}{l}2004 \\
\text { final }\end{array}$ & $\begin{array}{l}2005 \\
\text { final }\end{array}$ & $\begin{array}{c}2006 \\
\text { provisional } \\
\text { real-time }\end{array}$ \\
\hline 03/07/1990 & 9.1 & 1,790 & 4.35 & $X$ & -- & -- \\
\hline 04/17/1990 & 2.0 & 3,150 & 5.97 & $X$ & -- & -- \\
\hline 07/11/1990 & .3 & 3,100 & 6.33 & $X$ & -- & -- \\
\hline $11 / 07 / 1990$ & .52 & 2,650 & 5.82 & $X$ & -- & -- \\
\hline 02/13/1991 & 1.1 & 2,430 & 5.17 & $X$ & -- & -- \\
\hline 03/20/1991 & .86 & 2,580 & 5.86 & $\mathrm{X}$ & -- & -- \\
\hline 05/02/1991 & .96 & 2,890 & 6.21 & $X$ & -- & -- \\
\hline $12 / 19 / 1991$ & .49 & 3,300 & 5.86 & $\mathrm{X}$ & -- & -- \\
\hline 03/25/1992 & .49 & 2,500 & 5.47 & $X$ & -- & -- \\
\hline 05/05/1992 & .41 & 2,830 & 6.26 & $\mathrm{X}$ & -- & -- \\
\hline 06/24/1992 & .92 & 1,740 & 4.21 & $\mathrm{X}$ & -- & -- \\
\hline 11/11/1992 & .82 & 3,000 & 6.12 & $\mathrm{X}$ & -- & -- \\
\hline 03/24/1993 & 2.9 & 2,660 & 5.65 & $X$ & -- & -- \\
\hline 05/12/1993 & 1.4 & 3,170 & 6.32 & $\mathrm{X}$ & -- & -- \\
\hline 06/29/1993 & 1.0 & 2,770 & 6.38 & $X$ & -- & -- \\
\hline $10 / 05 / 1993$ & .05 & 2,200 & 5.96 & $X$ & -- & -- \\
\hline 01/05/1994 & .53 & 2,740 & 5.44 & $X$ & -- & -- \\
\hline 06/16/1994 & 1.0 & 3,280 & 6.83 & $X$ & -- & -- \\
\hline 07/26/1994 & .43 & 2,750 & 6.16 & $X$ & -- & -- \\
\hline $11 / 15 / 1994$ & .29 & 2,710 & 5.81 & $X$ & -- & -- \\
\hline 01/11/1995 & .07 & 2,720 & 5.52 & $X$ & -- & -- \\
\hline
\end{tabular}


Table 11. Water-sample data collected at Hanging Woman Creek near Birney, Montana (06307600), and used in regression equations for estimating sodium-adsorption ratio from specific conductance. - Continued

[Abbreviations: $\mathrm{ft}^{3} / \mathrm{s}$, cubic feet per second; SC, specific conductance; $\mu \mathrm{S} / \mathrm{cm}$, microsiemens per centimeter at 25 degrees Celsius; SAR, sodium-adsorption ratio; E, estimated value for streamflow. Symbol: --, data not used]

\begin{tabular}{|c|c|c|c|c|c|c|}
\hline \multirow[b]{2}{*}{ Date } & \multirow[b]{2}{*}{$\begin{array}{l}\text { Streamflow, } \\
\text { in } \mathrm{ft}^{3} / \mathrm{s}\end{array}$} & \multirow[b]{2}{*}{$\begin{array}{c}S C, \\
\text { in } \mu S / c m\end{array}$} & \multirow[b]{2}{*}{ SAR } & \multicolumn{3}{|c|}{$\begin{array}{l}\text { Sample data used for } \\
\text { regression equation }\end{array}$} \\
\hline & & & & $\begin{array}{l}2004 \\
\text { final }\end{array}$ & $\begin{array}{l}2005 \\
\text { final }\end{array}$ & $\begin{array}{c}2006 \\
\text { provisional } \\
\text { real-time }\end{array}$ \\
\hline 05/02/1995 & 2.6 & 3,290 & 6.25 & $X$ & -- & -- \\
\hline 07/20/1995 & 1.0 & 3,050 & 6.42 & $X$ & -- & -- \\
\hline 07/01/2003 & .34 & 2,640 & 5.80 & $X$ & -- & -- \\
\hline 08/05/2003 & .03 & 1,920 & 4.93 & $X$ & -- & -- \\
\hline $10 / 06 / 2003$ & E.02 & 1,650 & 3.20 & $X$ & $\mathrm{X}$ & $\mathrm{X}$ \\
\hline $11 / 03 / 2003$ & .01 & 1,880 & 3.74 & $X$ & $\mathrm{X}$ & $\mathrm{X}$ \\
\hline $12 / 04 / 2003$ & .08 & 2,370 & 4.77 & $X$ & $\mathrm{X}$ & $\mathrm{X}$ \\
\hline $02 / 05 / 2004$ & .04 & 3,410 & 6.00 & $X$ & $\mathrm{X}$ & $\mathrm{X}$ \\
\hline 03/10/2004 & E.09 & 2,000 & 4.42 & $X$ & $\mathrm{X}$ & $\mathrm{X}$ \\
\hline $04 / 13 / 2004$ & .25 & 2,720 & 4.86 & $\mathrm{X}$ & $\mathrm{X}$ & $\mathrm{X}$ \\
\hline $04 / 26 / 2004$ & .22 & 2,430 & 5.00 & $\mathrm{X}$ & $\mathrm{X}$ & $\mathrm{X}$ \\
\hline $05 / 12 / 2004$ & .22 & 2,210 & 4.65 & $X$ & $\mathrm{X}$ & $\mathrm{X}$ \\
\hline $05 / 25 / 2004$ & .54 & 2,570 & 5.32 & $\mathrm{X}$ & $\mathrm{X}$ & $\mathrm{X}$ \\
\hline 06/08/2004 & .14 & 1,880 & 5.04 & $\mathrm{X}$ & $\mathrm{X}$ & $\mathrm{X}$ \\
\hline $06 / 22 / 2004$ & .1 & 2,000 & 4.65 & $\mathrm{X}$ & $\mathrm{X}$ & $\mathrm{X}$ \\
\hline $10 / 13 / 2004$ & .04 & 1,660 & 3.12 & -- & $\mathrm{X}$ & $\mathrm{X}$ \\
\hline $12 / 02 / 2004$ & E. 1 & 2,300 & 4.12 & -- & $\mathrm{X}$ & $\mathrm{X}$ \\
\hline $02 / 08 / 2005$ & E.1 & 2,080 & 3.94 & -- & $\mathrm{X}$ & $\mathrm{X}$ \\
\hline 03/08/2005 & .32 & 1,760 & 3.72 & -- & $\mathrm{X}$ & $\mathrm{X}$ \\
\hline $04 / 05 / 2005$ & .32 & 2,130 & 4.35 & -- & $\mathrm{X}$ & $\mathrm{X}$ \\
\hline $04 / 19 / 2005$ & .25 & 2,190 & 4.41 & -- & $\mathrm{X}$ & $\mathrm{X}$ \\
\hline $05 / 02 / 2005$ & .22 & 2,280 & 4.69 & -- & $\mathrm{X}$ & $\mathrm{X}$ \\
\hline $05 / 16 / 2005$ & .54 & 2,300 & 4.81 & -- & $\mathrm{X}$ & $\mathrm{X}$ \\
\hline 06/08/2005 & .25 & 2,320 & 4.59 & -- & $\mathrm{X}$ & $\mathrm{X}$ \\
\hline $06 / 21 / 2005$ & .16 & 2,170 & 4.38 & -- & $\mathrm{X}$ & $\mathrm{X}$ \\
\hline $07 / 13 / 2005$ & .03 & 1,850 & 4.39 & -- & $\mathrm{X}$ & $\mathrm{X}$ \\
\hline
\end{tabular}




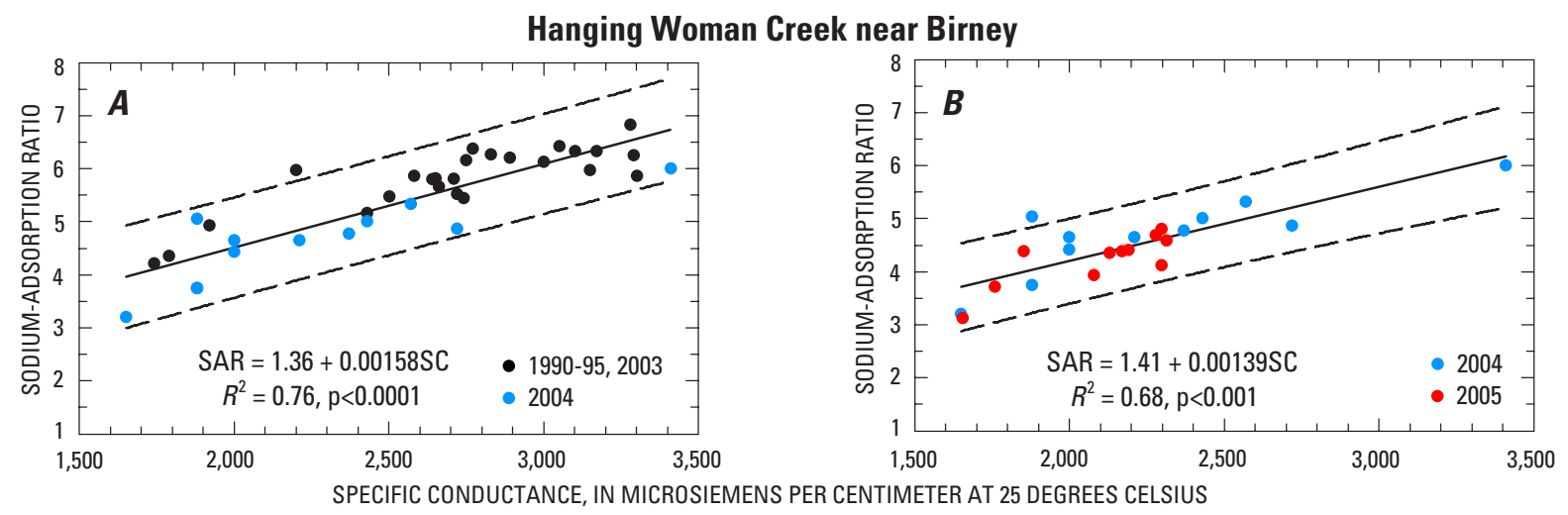

Figure 20. Relation of specific conductance (SC) and sodium-adsorption ratio (SAR) for water-quality samples collected at Hanging Woman Creek near Birney, Montana: $A$, Relation used to estimate SAR for the 2004 irrigation season and based on data collected March 1990 through June 2004; B, Relation used to estimate SAR for the 2005 irrigation season and based on data collected October 2003 through July 2005. Dashed lines indicate 95-percent prediction interval.
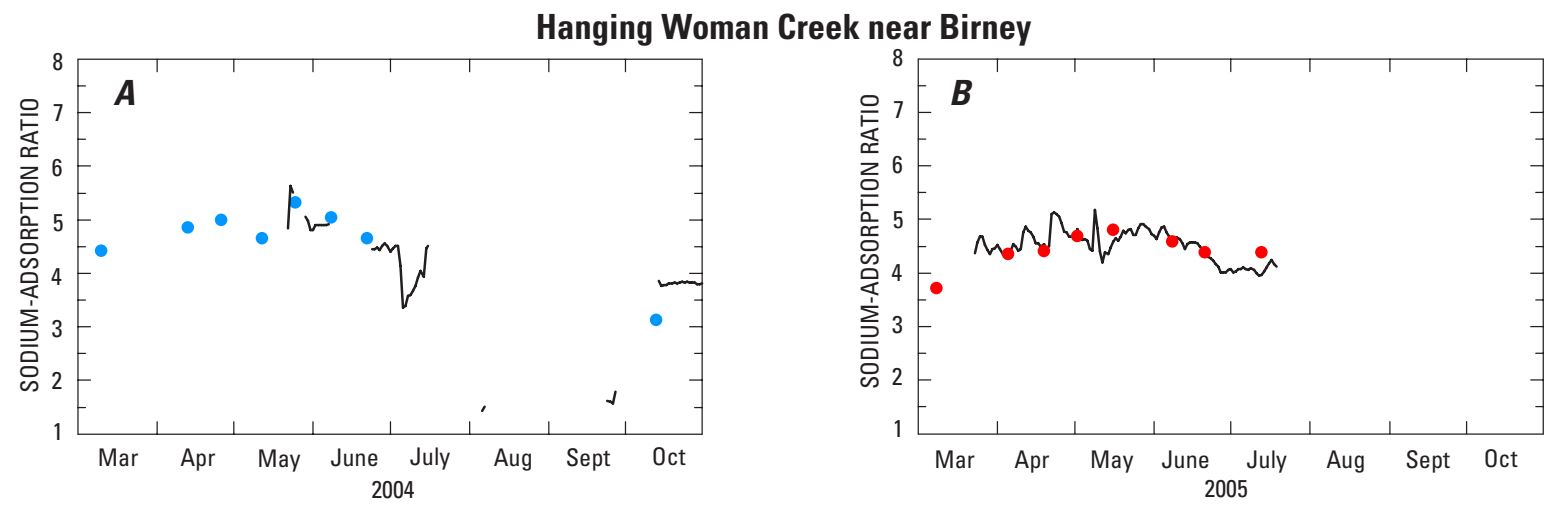

\section{EXPLANATION}

— Sodium-adsorption ratio (SAR) estimated from specific conductance (SC) • • Sodium-adsorption ratio (SAR) of samples

Figure 21. Sodium-adsorption ratio (SAR) determined from water-quality samples and estimated from specific conductance (SC) for Hanging Woman Creek near Birney, Montana, using relations shown in figure 20: $A, 2004$ irrigation season; $B, 2005$ irrigation season. 


\section{Otter Creek at Ashland, Montana}

This station (USGS station 06307740) is located in Rosebud County, Montana, downstream from bridge on U.S. Highway 212, and 0.3 mile southeast of Ashland (fig. 1). The station is at an altitude of 2,916.6 feet (NGVD 29) and has a drainage area of 707 square miles.

\section{Sodium-Adsorption Ratio Measured from Water Samples}

Fifty-two water samples were collected at this station from May 1990 through October 2005 (table 12). Samples were analyzed for major ions, nutrients, trace elements, and suspended sediment. Only values for instantaneous streamflow, SC, and SAR are shown; values for the remaining constituents can be accessed at http://waterdata.usgs.gov/mt/nwis.

\section{Estimated Sodium-Adsorption Ratio and Regression Equations for 2004-06}

SAR values for Otter Creek at Ashland, Montana, for the 2004 irrigation season were estimated using continuous SC data and a regression equation (fig. 22A) based on the $\mathrm{SC}$ and SAR values from 39 water samples collected from May 1990 through August 2004 (table 12). SAR values for the 2005 irrigation season were estimated using continuous SC and a regression equation (fig. 22B) based on data from 24 samples collected from December 2003 through October 2005 (table 12). Provisional real-time SAR data for 2006 also were estimated using the equation for the 2005 irrigation season (fig. 22B). Comparisons of daily mean estimated SAR for the 2004 and 2005 irrigation seasons are shown on figure 23.

Table 12. Water-sample data collected at Otter Creek at Ashland, Montana (06307740), and used in regression equations for estimating sodium-adsorption ratio from specific conductance.

[Abbreviations: $\mathrm{ft}^{3} / \mathrm{s}$, cubic feet per second; $\mathrm{SC}$, specific conductance; $\mu \mathrm{S} / \mathrm{cm}$, microsiemens per centimeter at 25 degrees Celsius; SAR, sodium-adsorption ratio; E, estimated value for streamflow. Symbol: --, data not used]

\begin{tabular}{|c|c|c|c|c|c|c|}
\hline \multirow[b]{2}{*}{ Date } & \multirow{2}{*}{$\begin{array}{c}\text { Streamflow, } \\
\mathrm{ft}^{3} / \mathrm{s}\end{array}$} & \multirow{2}{*}{$\begin{array}{c}\mathrm{SC}, \\
\mu \mathrm{S} / \mathrm{cm}\end{array}$} & \multirow[b]{2}{*}{ SAR } & \multicolumn{3}{|c|}{$\begin{array}{l}\text { Sample data used for } \\
\text { regression equation }\end{array}$} \\
\hline & & & & $\begin{array}{l}2004 \\
\text { final }\end{array}$ & $\begin{array}{l}2005 \\
\text { final }\end{array}$ & $\begin{array}{c}2006 \\
\text { provisional } \\
\text { real-time }\end{array}$ \\
\hline 05/10/1990 & 1.8 & 2,880 & 6.51 & $X$ & -- & -- \\
\hline 06/25/1990 & 1.9 & 3,000 & 6.34 & $X$ & -- & -- \\
\hline $09 / 27 / 1990$ & .29 & 2,920 & 6.44 & $X$ & -- & -- \\
\hline $10 / 18 / 1990$ & .49 & 2,650 & 6.47 & $X$ & -- & -- \\
\hline 03/21/1991 & 1.2 & 2,100 & 5.26 & $\mathrm{X}$ & -- & -- \\
\hline 05/31/1991 & 1.1 & 2,640 & 6.37 & $X$ & -- & -- \\
\hline 09/23/1991 & .41 & 2,700 & 6.74 & $X$ & -- & -- \\
\hline 01/13/1992 & .71 & 3,290 & 5.44 & $\mathrm{X}$ & -- & -- \\
\hline 05/04/1992 & .71 & 2,830 & 6.59 & $X$ & -- & -- \\
\hline 06/04/1992 & .5 & 2,920 & 6.52 & $X$ & -- & -- \\
\hline 07/30/1992 & .08 & 2,790 & 6.50 & $\mathrm{X}$ & -- & -- \\
\hline $12 / 08 / 1992$ & .51 & 3,030 & 6.43 & $X$ & -- & -- \\
\hline 04/13/1993 & 2.1 & 2,690 & 5.69 & $X$ & -- & -- \\
\hline 06/01/1993 & .77 & 3,160 & 6.59 & $X$ & -- & -- \\
\hline 08/11/1993 & 1.1 & 2,960 & 6.59 & $X$ & -- & -- \\
\hline $10 / 06 / 1993$ & .61 & 2,660 & 5.91 & $X$ & -- & -- \\
\hline 01/05/1994 & 1.2 & 2,670 & 5.55 & $X$ & -- & -- \\
\hline 06/17/1994 & 2.8 & 3,330 & 5.93 & $X$ & -- & -- \\
\hline 07/27/1994 & .93 & 3,030 & 6.36 & $X$ & -- & -- \\
\hline 10/05/1994 & 1.0 & 2,500 & 5.86 & $X$ & -- & -- \\
\hline
\end{tabular}


Table 12. Water-sample data collected at Otter Creek at Ashland, Montana (06307740), and used in regression equations for estimating sodium-adsorption ratio from specific conductance.-Continued

[Abbreviations: $\mathrm{ft}^{3} / \mathrm{s}$, cubic feet per second; SC, specific conductance; $\mu \mathrm{S} / \mathrm{cm}$, microsiemens per centimeter at 25 degrees Celsius; SAR, sodium-adsorption ratio; E, estimated value for streamflow. Symbol: --, data not used]

\begin{tabular}{|c|c|c|c|c|c|c|}
\hline \multirow[b]{2}{*}{ Date } & \multirow{2}{*}{$\begin{array}{c}\text { Streamflow, } \\
\mathrm{ft}^{3} / \mathrm{s}\end{array}$} & \multirow{2}{*}{$\begin{array}{c}S C, \\
\mu S / c m\end{array}$} & \multirow[b]{2}{*}{ SAR } & \multicolumn{3}{|c|}{$\begin{array}{l}\text { Sample data used for } \\
\text { regression equation }\end{array}$} \\
\hline & & & & $\begin{array}{l}2004 \\
\text { final }\end{array}$ & $\begin{array}{l}2005 \\
\text { final }\end{array}$ & $\begin{array}{c}2006 \\
\text { provisional } \\
\text { real-time }\end{array}$ \\
\hline $01 / 12 / 1995$ & 1.1 & 2,610 & 5.52 & $\mathrm{X}$ & -- & -- \\
\hline 06/14/1995 & 5.6 & 3,260 & 6.38 & $X$ & -- & -- \\
\hline 07/20/1995 & 6.0 & 1,040 & 3.08 & $X$ & -- & -- \\
\hline $07 / 02 / 2003$ & 1.73 & 3,120 & 6.19 & $\mathrm{X}$ & -- & -- \\
\hline 08/05/2003 & .65 & 2,840 & 6.64 & $\mathrm{X}$ & -- & -- \\
\hline $09 / 02 / 2003$ & .08 & 2,480 & 6.07 & $X$ & -- & -- \\
\hline $10 / 07 / 2003$ & E.55 & 2,210 & 5.22 & $\mathrm{X}$ & -- & -- \\
\hline $11 / 13 / 2003$ & .84 & 2,200 & 4.93 & $X$ & -- & -- \\
\hline $12 / 03 / 2003$ & E1.0 & 2,740 & 6.01 & $\mathrm{X}$ & $\mathrm{X}$ & $\mathrm{X}$ \\
\hline $02 / 03 / 2004$ & E.75 & 2,635 & 5.86 & $\mathrm{X}$ & $\mathrm{X}$ & $\mathrm{X}$ \\
\hline 03/10/2004 & E1.5 & 1,960 & 4.80 & $\mathrm{X}$ & $\mathrm{X}$ & $\mathrm{X}$ \\
\hline $04 / 14 / 2004$ & 4.7 & 2,760 & 6.08 & $\mathrm{X}$ & $\mathrm{X}$ & $\mathrm{X}$ \\
\hline $04 / 26 / 2004$ & E1.2 & 2,815 & 6.05 & $\mathrm{X}$ & $X$ & $\mathrm{X}$ \\
\hline $05 / 12 / 2004$ & E. 5 & 2,870 & 6.75 & $\mathrm{X}$ & $\mathrm{X}$ & $\mathrm{X}$ \\
\hline $05 / 24 / 2004$ & 1.79 & 2,330 & 5.63 & $\mathrm{X}$ & $\mathrm{X}$ & $\mathrm{X}$ \\
\hline 06/08/2004 & E.75 & 2,930 & 6.66 & $X$ & $\mathrm{X}$ & $\mathrm{X}$ \\
\hline $06 / 23 / 2004$ & E1.1 & 2,670 & 6.44 & $\mathrm{X}$ & $\mathrm{X}$ & $\mathrm{X}$ \\
\hline $07 / 13 / 2004$ & E1.0 & 2,690 & 6.54 & $\mathrm{X}$ & $\mathrm{X}$ & $\mathrm{X}$ \\
\hline $08 / 18 / 2004$ & E. 4 & 2,390 & 5.64 & $X$ & $X$ & $\mathrm{X}$ \\
\hline $10 / 12 / 2004$ & E.15 & 2,263 & 5.33 & -- & $\mathrm{X}$ & $\mathrm{X}$ \\
\hline $12 / 08 / 2004$ & 1.7 & 2,949 & 6.11 & -- & $X$ & $\mathrm{X}$ \\
\hline $02 / 09 / 2005$ & 1.6 & 2,500 & 5.43 & -- & $\mathrm{X}$ & $\mathrm{X}$ \\
\hline 03/08/2005 & 1.8 & 2,340 & 5.37 & -- & $\mathrm{X}$ & $\mathrm{X}$ \\
\hline $04 / 05 / 2005$ & 1.7 & 2,990 & 6.54 & -- & $\mathrm{X}$ & $\mathrm{X}$ \\
\hline $04 / 18 / 2005$ & 1.8 & 2,910 & 6.85 & -- & $\mathrm{X}$ & $\mathrm{X}$ \\
\hline $05 / 04 / 2005$ & 2.1 & 2,810 & 6.32 & -- & $X$ & $\mathrm{X}$ \\
\hline $05 / 16 / 2005$ & 9.3 & 2,550 & 5.82 & -- & $\mathrm{X}$ & $\mathrm{X}$ \\
\hline 06/07/2005 & 2.6 & 2,890 & 6.12 & -- & $\mathrm{X}$ & $\mathrm{X}$ \\
\hline $06 / 21 / 2005$ & 2.2 & 3,180 & 6.22 & -- & $X$ & $\mathrm{X}$ \\
\hline $07 / 12 / 2005$ & .96 & 2,810 & 6.22 & -- & $\mathrm{X}$ & $X$ \\
\hline $08 / 02 / 2005$ & 1.0 & 2,330 & 5.45 & -- & $X$ & $X$ \\
\hline $10 / 04 / 2005$ & 1.0 & 2,130 & 5.12 & -- & $X$ & $\mathrm{X}$ \\
\hline
\end{tabular}




\section{Otter Creek at Ashland}
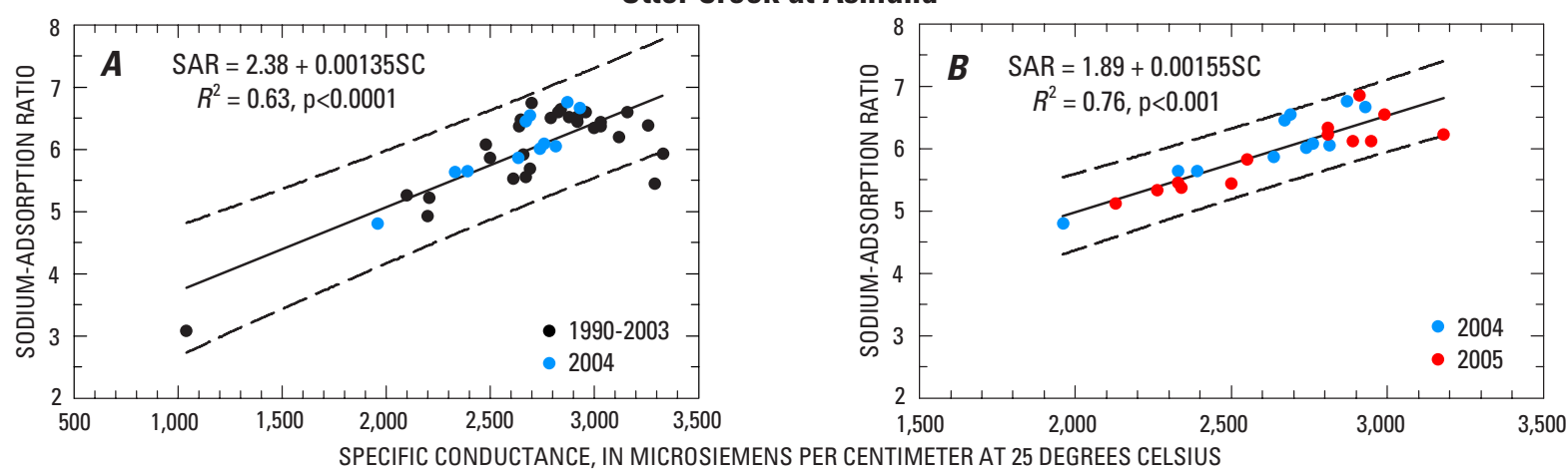

Figure 22. Relation of specific conductance (SC) and sodium-adsorption ratio (SAR) for water-quality samples collected at Otter Creek at Ashland, Montana: $A$, Relation used to estimate SAR for the 2004 irrigation season and based on data collected May 1990 through August 2004; B, Relation used to estimate SAR for the 2005 irrigation season and based on data collected December 2003 through October 2005. Dashed lines indicate 95-percent prediction interval.

Otter Creek at Ashland
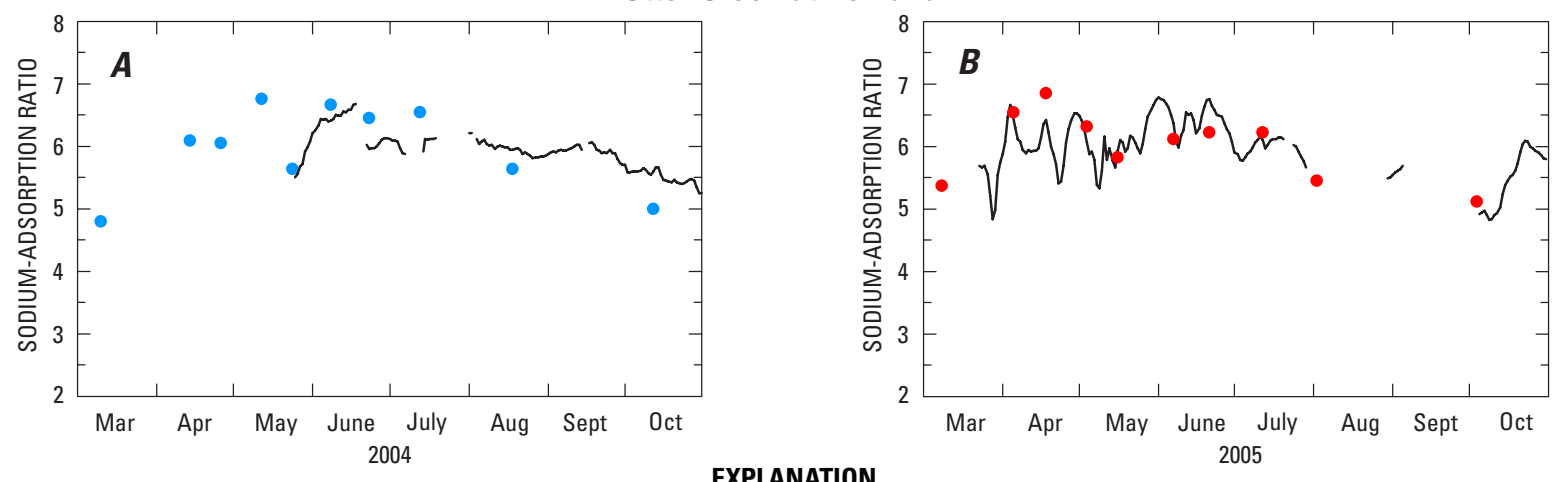

EXPLANATION

— Sodium-adsorption ratio (SAR) estimated from specific conductance (SC) $\bullet$ Sodium-adsorption ratio (SAR) of samples

Figure 23. Sodium-adsorption ratio (SAR) determined from water-quality samples and estimated from specific conductance (SC) for Otter Creek at Ashland, Montana, using relations shown in figure 22: A, 2004 irrigation season; $B, 2005$ irrigation season. 


\section{Pumpkin Creek near Miles City, Montana}

This station (USGS station 06308400) is located in Custer County, Montana, 16 miles southeast of Miles City (fig. 1). The station is at an altitude of 2,476 feet (NGVD 29) and has a drainage area of 697 square miles.

\section{Sodium-Adsorption Ratio Measured from Water Samples}

Seventeen water samples were collected at this station from March 2004 through October 2005 (table 13). Samples were analyzed for major ions, nutrients, trace elements, and suspended sediment. Only values for instantaneous streamflow, SC, and SAR are shown; values for the remaining constituents can be accessed at $h t t p: / /$ waterdata.usgs.gov/mt/nwis.

SAR values for the 2004 and 2005 irrigation seasons were not estimated from SC data because the relations between SC and SAR derived from the sample data were not statistically significant. Also, provisional real-time SAR values were not estimated for 2006. A plot of SC and SAR values for water-quality samples collected from March 2004 through October 2005 is shown on figure 24 to illustrate the range of values and lack of a discernible relation.

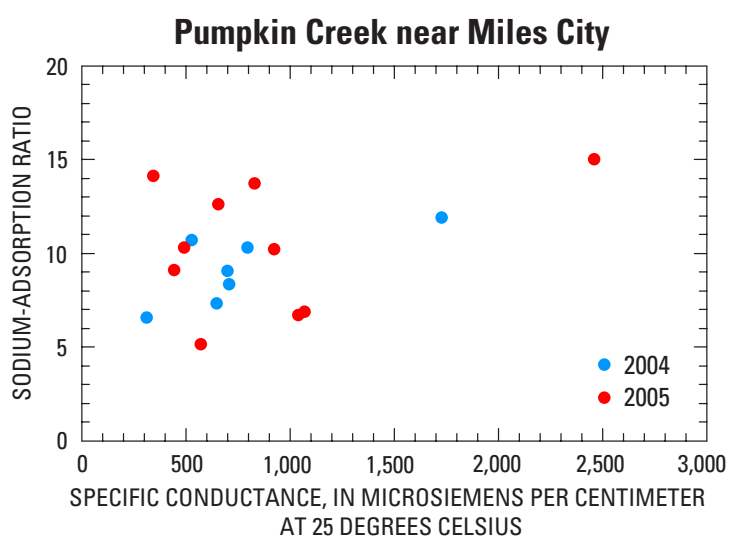

Figure 24. Specific conductance (SC) and sodiumadsorption ratio (SAR) for water-quality samples collected March 2004 through October 2005 at Pumpkin Creek near Miles City, Montana (estimated SAR values are not available for this site for 2004-06).

Table 13. Water-sample data collected at Pumpkin Creek near Miles City, Montana (06308400), and used in regression analysis of specific conductance and sodium-adsorption ratio.

[The regression relation developed from these sample data was not statistically significant; therefore, SAR was not estimated for 2004-06. Abbreviations: $\mathrm{ft}^{3} / \mathrm{s}$, cubic feet per second; SC, specific conductance; $\mu \mathrm{S} / \mathrm{cm}$, microsiemens per centimeter at 25 degrees Celsius; SAR, sodium-adsorption ratio]

\begin{tabular}{cccc}
\hline Date & $\begin{array}{c}\text { Streamflow, } \\
\text { in ft } \mathbf{3}^{\mathbf{s}}\end{array}$ & $\begin{array}{c}\text { SC, } \\
\text { in } \boldsymbol{\mu S} / \mathbf{c m}\end{array}$ & SAR \\
\hline $03 / 10 / 2004$ & 6.2 & 647 & 7.32 \\
$05 / 24 / 2004$ & 121 & 311 & 6.55 \\
$06 / 16 / 2004$ & .72 & 700 & 9.02 \\
$07 / 06 / 2004$ & 89.5 & 797 & 10.32 \\
$07 / 13 / 2004$ & 16 & 706 & 8.33 \\
$09 / 28 / 2004$ & .12 & 1,730 & 11.88 \\
$11 / 02 / 2004$ & 2.49 & 526 & 10.75 \\
$04 / 06 / 2005$ & .06 & 2,460 & 15.01 \\
$04 / 11 / 2005$ & 9.7 & 493 & 10.34 \\
$04 / 19 / 2005$ & .23 & 830 & 13.75 \\
$04 / 21 / 2005$ & 30 & 654 & 12.57 \\
$05 / 04 / 2005$ & .05 & 925 & 10.18 \\
$05 / 09 / 2005$ & 197 & 442 & 9.07 \\
$06 / 08 / 2005$ & 584 & 570 & 5.12 \\
$06 / 22 / 2005$ & 1.45 & 1,040 & 6.69 \\
$07 / 13 / 2005$ & .23 & 1,070 & 6.85 \\
$10 / 05 / 2005$ & .15 & 345 & 14.11 \\
\hline
\end{tabular}




\section{Summary}

The northward-flowing Tongue River drains an area of about 5,400 square miles in the coal-rich areas of northeastern Wyoming and southeastern Montana. Since about 1999, coalbed methane (CBM) resources underlying the Tongue River watershed near the border of Wyoming and Montana have been a major focus of energy exploration and development. Production of CBM requires the pumping of large volumes of ground water from the coal beds to reduce water pressure within the formation and release the stored gas. Disposal of ground water with high concentrations of sodium into the Tongue River has the potential to increase salinity and sodium adsorption ratio (SAR) of water in the river, and potentially reduce the quality of water for irrigation purposes.

Starting in 2004, Congress appropriated funding for the U.S. Geological Survey (USGS) to initiate and operate a comprehensive surface-water-quality monitoring program in the Tongue River watershed to describe current conditions and potential changes in streams. A monitoring network consisting of 12 water-quality stations was established by the USGS during 2004 and 2005 with support from the Congressional funding and cooperative programs with several Federal, State, Tribal, and local entities.

The purpose of this report is to document measured and estimated SAR for Tongue River and its tributaries in Montana and Wyoming. Specific conductance (SC) and SAR values were measured in water samples collected at the 12 monitoring sites in the Tongue River watershed. In addition, regression relations between SC and SAR were analyzed for each site for the years 2004-06 and appropriate regression equations were developed for estimating SAR from SC. The results of regression analysis indicated that SC and SAR were significantly related ( $p$-values $<0.05$ ) at most sites. The regression equations developed for most monitoring sites in the Tongue River watershed were used with the continuous SC data to estimate daily SAR values during the 2004 and 2005 irrigation seasons (generally March-October) and to estimate 2006 provisional SAR values.

SAR in water samples was determined from laboratorymeasured concentrations of sodium, calcium, and magnesium in water samples. Water samples were collected under various flow conditions and at different times of the year in an attempt to obtain samples that reflected seasonal and annual changes in water quality. The 12 sites monitored in the Tongue River watershed include 7 sites on the mainstem of the Tongue River and 5 sites on major tributaries to the Tongue River. The 7 sites on the mainstem of the Tongue River are located at: (1) Monarch, Wyoming, station 06299980, (2) State line near Decker, Montana, station 06306300, (3) Tongue River Dam near Decker, Montana, station 06307500, (4) Birney Day School Bridge near Birney, Montana, station 06307616, (5) below Brandenberg Bridge near Ashland, Montana, station 06307830, (6) above T\&Y Diversion Dam near Miles City, Montana, station 06307990, and (7) Miles City, Mon- tana, station 06308500. The five sites on tributaries are located at: (1) Goose Creek near Acme, Wyoming, station 06305700, (2) Prairie Dog Creek near Acme, Wyoming, station 06306250, (3) Hanging Woman Creek near Birney, Montana, station 06307600, (4) Otter Creek at Ashland, Montana, station 06307740, and (5) Pumpkin Creek near Miles City, Montana, station 06308400.

Regression equations for estimating SAR at Tongue River at Monarch, Wyoming, were based on SC and SAR of 32 water samples collected from January 2004 through October 2005. Two equations were developed: one for estimating SAR during the 2004 irrigation season and the second for estimating SAR during the 2005 irrigation season. Provisional SAR during 2006 also was estimated using the 2005 equation. The equation for 2004 was based on data from 17 of the samples, and the equation for 2005-06 was based on data from all 32 samples.

Regression equations for estimating SAR at Tongue River at State line, near Decker, Montana, were based on SC and SAR of 83 water samples collected from October 1999 through October 2005. Two equations were developed: one for estimating SAR during the 2004 irrigation season and the second for estimating SAR during the 2005 irrigation season. Provisional SAR during 2006 also was estimated using the 2005 equation. The equation for 2004 was based on data from 62 of the samples, and the equation for 2005-06 was based on data from 39 water samples.

Thirty-six water samples were collected at Tongue River at Tongue River Dam, near Decker, Montana, from January 2004 through October 2005. SAR was not estimated for the 2004 irrigation season because the regression relation based on SC and SAR from 17 water samples collected from January through September 2004 was not statistically significant. A regression equation was developed for estimating SAR during the 2005 irrigation season, based on SC and SAR of all 36 water samples. Provisional SAR during 2006 also was estimated using the 2005 equation.

Thirty-six water samples were collected at Tongue River at Birney Day School Bridge, near Birney, Montana, from January 2004 through October 2005. SAR was not estimated for the 2004 irrigation season because the regression equation based on SC and SAR from 17 water samples collected from January through September 2004 was not statistically significant. A regression equation was developed for estimating SAR during the 2005 irrigation season, based on SC and SAR of all 36 water samples. Provisional SAR during 2006 also was estimated using the 2005 equation.

Regression equations for estimating SAR at Tongue River below Brandenberg Bridge, near Ashland, Montana, were based on SC and SAR of 78 water samples collected from June 2000 through October 2005. Two equations were developed: one for estimating SAR during the 2004 irrigation season and the second for estimating SAR during the 2005 irrigation season. Provisional SAR during 2006 also was estimated using the 2005 equation. The equation for 2004 was 
based on data from 59 of the samples, and the equation for 2005-06 was based on data from 35 water samples.

Twenty water samples were collected at Tongue River above T\&Y Diversion Dam, near Miles City, Montana, from November 2004 through October 2005. SAR was not estimated for the 2004 irrigation season because only two samples were collected in 2004. A regression equation was developed for estimating SAR during the 2005 irrigation season, based on SC and SAR of all 20 water samples. Provisional SAR during 2006 also was estimated using the 2005 equation.

Regression equations for estimating SAR at Tongue River at Miles City, Montana, were based on SC and SAR of 62 water samples collected from February 1990 through October 2005. Two equations were developed: one for estimating SAR during the 2004 irrigation season and the second for estimating SAR during the 2005 irrigation season. Provisional SAR during 2006 also was estimated using the 2005 equation. The equation for 2004 was based on data from 42 of the samples, and the equation for 2005-06 was based on data from 33 water samples.

Regression equations for estimating SAR at Goose Creek near Acme, Wyoming, were based on SC and SAR of 23 water samples collected from February 2004 through October 2005. Two equations were developed: one for estimating SAR during the 2004 irrigation season and the second for estimating SAR during the 2005 irrigation season. Provisional SAR during 2006 also was estimated using the 2005 equation. The equation for 2004 was based on data from 10 of the samples and the equation for 2005-06 was based on data from all 23 water samples.

Regression equations for estimating SAR at Prairie Dog Creek near Acme, Wyoming, were based on SC and SAR of 68 water samples collected from May 2000 through October 2005. Two equations were developed: one for estimating SAR during the 2004 irrigation season and the second for estimating SAR during the 2005 irrigation season. Provisional SAR during 2006 also was estimated using the 2005 equation. The regression equation for 2004 was based on data from 33 samples and the regression equation for 2005-06 was based on data from 28 water samples.

Regression equations for estimating SAR at Hanging Woman Creek near Birney, Montana, were based on SC and SAR of 47 water samples collected from March 1990 through July 2005. Two equations were developed: one for estimating SAR during the 2004 irrigation season and the second for estimating SAR during the 2005 irrigation season. Provisional SAR during 2006 also was estimated using the 2005 equation. The equation for 2004 was based on data from 36 of the samples, and the equation for 2005-06 was based on data from 22 water samples.

Regression equations for estimating SAR at Otter Creek at Ashland, Montana, were based on SC and SAR of 52 water samples collected from May 1990 through October 2005. Two equations were developed: one for estimating SAR during the 2004 irrigation season and the second for estimating SAR during the 2005 irrigation season. Provisional SAR during 2006 also was estimated using the 2005 equation. The equation for 2004 was based on data from 39 of the samples, and the equation for 2005-06 was based on data from 24 water samples.

Seventeen water samples were collected at Pumpkin Creek near Miles City, Montana, from March 2004 through October 2005. SAR was not estimated for Pumpkin Creek because the relation between SC and SAR was not statistically significant.

\section{References Cited}

Berkas, W.R., White, M.K., Ladd, P.B., Bailey, F.A., and Dodge, K.A., 2006, Water resources data, Montana, water year 2005, v. 2. Yellowstone and upper Columbia River basins and ground-water levels: U.S. Geological Survey Water-Data Report MT-05-2, 561 p.

Cannon, M.R., and Johnson, D.R., 2004, Estimated water use in Montana in 2000: U.S. Geological Survey Scientific Investigations Report 2004-5223, 50 p.

Dodge, K.A., and Lambing, J.H., 2006, Quality-assurance plan for the analysis of suspended sediment by the U.S. Geological Survey in Montana: U.S. Geological Survey Open-File Report 2006-1242, 25 p., accessed March 14, 2007, at http://pubs.er.usgs.gov/usgspubs/ofr/ofr20061242

Edwards, T.K., and Glysson, G.D., 1999, Field methods for measurement of fluvial sediment: U.S. Geological Survey Techniques of Water-Resources Investigations, book 3, chap. C2, 89 p.

Fishman, M.J., ed., 1993, Methods of analysis by the U.S. Geological Survey National Water Quality LaboratoryDetermination of inorganic and organic constituents in water and fluvial sediments: U.S. Geological Survey OpenFile Report 93-125, 217 p.

Fishman, M.J., and Friedman, L.C., eds., 1989, Methods for determination of inorganic substances in water and fluvial sediments: U.S. Geological Survey Techniques of WaterResources Investigations, book 5, chap. A1, 545 p.

Friedman, L.C., and Erdmann, D.E., 1982, Quality assurance practices for the chemical and biological analyses of water and fluvial sediments: U.S. Geological Survey Techniques of Water-Resources Investigations, book 5, chap. A6, 181 p., accessed March 14, 2007, at http://pubs.usgs. gov/twril

Garbarino, J.R., and Struzeski, T.M., 1998, Methods of analysis by the U.S. Geological Survey National Water Quality Laboratory-Determination of elements in whole-water digests using inductively coupled plasma-optical emission spectrometry and inductively coupled plasma-mass spectrometry: U.S. Geological Survey Open-File Report 98-165, $101 \mathrm{p}$. 
Guy, H.P., 1969, Laboratory theory and methods for sediment analysis: U.S. Geological Survey Techniques of WaterResources Investigations, book 5, chap. C1, 58 p.

Helsel, D.R., and Hirsch, R.M., 1992, Statistical methods in water resources: Amsterdam, Elsevier, 522 p.

Hem, J.D., 1985, Study and interpretation of the chemical characteristics of natural water ( $3 \mathrm{~d}$ ed.): U.S. Geological Survey Water-Supply Paper 2254, 264 p.

Hoffman, G.L., Fishman, M.J., and Garbarino, J.R., 1996, Methods of analysis by the U.S. Geological Survey National Water-Quality Laboratory-In-bottle acid digestion of whole-water samples: U.S. Geological Survey Open-File Report 96-225, 28 p.

Horowitz, A.J., Demas, C.R., Fitzgerald, K.K., Miller, T.L., and Rickert, D.A., 1994, U.S. Geological Survey protocol for the collection and processing of surface-water samples for the subsequent determination of inorganic constituents in filtered water: U.S. Geological Survey Open-File Report 94-539, $57 \mathrm{p}$.

Jones, B.E., 1987, Quality control manual of the U.S. Geological Survey's National Water Quality Laboratory: U.S. Geological Survey Open-File Report 87-457, 17 p.

Jones, S.R., and Garbarino, J.R., 1999, Methods of analysis by the U.S. Geological Survey National Water Quality Laboratory-Determination of arsenic and selenium in water and sediment by graphite furnace-atomic adsorption spectrometry: U.S. Geological Survey Open-File Report 98-639, 39 p.

Knapton, J.R., and Nimick, D.A., 1991, Quality assurance for water-quality activities of the U.S. Geological Survey in Montana: U.S. Geological Survey Open-File Report 91-216, $41 \mathrm{p}$.

McLain, B.J., 1993, Methods of analysis by the U.S. Geological Survey National Water Quality Laboratory-Determination of chromium in water by graphite furnace-atomic adsorption spectrophotometry: U.S. Geological Survey Open-File Report 93-449, 16 p.
Montana Department of Environmental Quality, 2003, Numeric standards for electrical conductivity (EC) and sodium adsorption ratio (SAR), Rule 17.30.670, Administrative rules of Montana, chapter 30, sub-chapter 6, p. 17-2757 to 17-2758, accessed March 14, 2007, at http://deq.state.mt.us/dir/legal/Chapters/CH30-06.pdf

Pritt, J.W., and Raese, J.W., eds., 1995, Quality assurance/ quality control manual-National Water Quality Laboratory: U.S. Geological Survey Open-File Report 95-443, $35 \mathrm{p}$.

Rantz, S.E., and others, 1982, Measurement and computation of streamflow: U.S. Geological Survey Water-Supply Paper 2175, 2 v., 631 p.

U.S. Geological Survey, variously dated, National field manual for the collection of water-quality data: U.S. Geological Survey Techniques of Water-Resources Investigations, book 9, chaps. A1-A9, available online at http://pubs.water. usgs.gov/twri9A/

Van Voast, W.A., 2003, Geochemical signature of formation waters associated with coalbed methane: American Association of Petroleum Geologists Bulletin, v. 87, no. 4, p. 667-676.

Wagner, R.J., Boulger, R.W., Jr., Oblinger, C.J., and Smith, B.A., 2006, Guidelines and standard procedures for continuous water-quality monitors-Station operation, record computation, and data reporting: U.S. Geological Survey Techniques and Methods 1-D3, 51 p., accessed March 14, 2007, at http://pubs.water.usgs.gov/tm1D3

Ward, J.R., and Harr, C.A., eds., 1990, Methods for collection and processing of surface-water and bed-material samples for physical and chemical analyses: U.S. Geological Survey Open-File Report 90-140, 71 p. 
Prepared by the Helena Publishing Service Center

For additional information contact:

Director, Montana Water Science Center

U.S. Geological Survey

3162 Bozeman Avenue

Helena, Montana 59601

(406) 457-5900

http://mt.water.usgs.gov/ 
